\title{
Human enteric viruses shape disease phenotype through divergent innate immunomodulation.
}

Authors: Fatemeh Adiliaghdam ${ }^{1}$, Hajera Amatullah ${ }^{1}$, Sreehaas Digumarthi ${ }^{1}$, Tahnee L. Saunders ${ }^{1}$, Raza-Ur Rahman ${ }^{1}$, Lai Ping Wong ${ }^{2,3}$, Ruslan Sadreyev ${ }^{2,4}$, Lindsay Droit ${ }^{5}$, Jean

Paquette $^{6}$, Philippe Goyette ${ }^{6}$, John Rioux ${ }^{6,7}$, Richard Hodin ${ }^{8}$, Kathie A. Mihindukulasuriya ${ }^{5}$, Scott A. Handley ${ }^{5}$, Kate L. Jeffrey ${ }^{1,9^{*}}$

\section{Affiliations:}

${ }^{1}$ Department of Medicine, Division of Gastroenterology and the Center for the Study of Inflammatory Bowel Disease, Massachusetts General Hospital, Harvard Medical School, Boston, 10 MA 02114, USA.

${ }^{2}$ Department of Molecular Biology, Massachusetts General Hospital, Harvard Medical School, Boston, MA 02114, USA.

$15{ }^{3}$ Department of Genetics, Massachusetts General Hospital, Harvard Medical School, Boston, MA 02114, USA

${ }^{4}$ Department of Pathology, Massachusetts General Hospital, Harvard Medical School, Boston, MA 02114, USA

${ }^{5}$ Department of Pathology and Immunology, Washington University School of Medicine, St Louis, MO, USA

${ }^{6}$ Montreal Heart Institute, Montreal Quebec Canada H1T 1C8

${ }^{7}$ Université de Montréal, Montreal Quebec Canada H3C 3J7

${ }^{8}$ Department of Surgery, Massachusetts General Hospital, Harvard Medical School, Boston, MA 02114, USA

${ }^{9}$ Center for Microbiome Informatics and Therapeutics, Massachusetts Institute of Technology, Cambridge, MA 02139, USA.

$35 *$ Correspondence to: KJeffrey@mgh.harvard.edu 


\section{Summary:}

Altered enteric microorganisms in concert with host genetics shape inflammatory bowel disease (IBD) phenotypes. However, insight is limited to bacteria and fungi. We found virus like particles (VLPs) enriched from normal human colon resections, containing eukaryotic viruses and bacteriophages (collectively, the virome), actively elicited atypical anti-inflammatory innate immune programs. Conversely, IBD patient VLPs provoked inflammation, which was successfully dampened by healthy VLPs. The IBD colon tissue virome was perturbed, including enriched Picornovirus Enterovirus B, not previously observed in fecal virome studies. Mice with humanized healthy colon tissue viromes had attenuated intestinal inflammation while those with IBD-derived viromes exhibited exacerbated inflammation in a nucleic acid sensing-dependent fashion. Furthermore, there were detrimental consequences for IBD-associated MDA5 loss-offunction on patient intestinal epithelial cells exposed to healthy or IBD viromes. Our results demonstrate that innate recognition of either healthy or IBD human viromes autonomously influences disease phenotypes in IBD. Harnessing the virome may offer therapeutic and biomarker potential.

One Sentence Summary: Human viromes divergently shape host immunity and disease

\section{Main Text:}

Viruses are the most abundant biological entity on earth and infect all types of life. Generally considered obligate pathogens, viruses are a constant and emerging threat to human health, as exemplified by the Severe Acute Respiratory Syndrome Coronavirus 2 (SARS-CoV-2) pandemic. Yet, paradoxically, many viruses persistently reside within asymptomatic individuals. These include a range of eukaryotic viruses, as well as bacteriophages (1-4), that exist both within their bacterial host, as well as free virions embedded within mucus layers of the intestine (5). These collective resident viruses, along with bacteria, archaea, fungi, helminths and protozoa form the human metagenome that have intimate functional relationships with their host. The human intestinal virome is established at birth and is dominated by bacteriophages, while eukaryotic viruses gradually emerge after birth, as measured in feces that contains $\sim 10^{9}$ virus like particles (VLPs) per gram $(2,6)$. Furthermore, despite multiple remaining hurdles in identifying what constitutes a normal human virome (7), robust fluctuations of the virome have been reported in $\operatorname{IBD}(1,8-10)$, colorectal cancer $(11,12)$, type I diabetes $(13)$, nonalcoholic fatty liver disease $(14)$, cystic fibrosis (15), graft-versus-host-disease (16) and HIV infection (17) and a range of other diseases (18). However, this rapid cataloging of viral genomes in human tissues by sequencing has vastly outpaced any mechanistic understanding we have of virome biology. Viruses inhabiting a healthy host $(19,20)$, and host innate sensing of viruses by gut-resident Toll-like Receptors (TLRs) or RIG-I like Receptors (RLRs) (19, 21-26) provide homeostasis in the mouse intestine. However, functional studies of the human healthy or diseased enteric virome are currently lacking. Although, examples of direct and distinct bacteriophage-human immune interactions are emerging $(5,27$, 28). Furthermore, loss-of-function variants in the gene $I F I H 1$ that encodes the host virus receptor melanoma differentiation associated protein 5 (MDA5) were shown to significantly associate with IBD by genome wide-association studies, GWAS (29). However, how abrogation of MDA5 function may contribute to intestinal inflammation in IBD, especially in the context of the virome, 45 is unclear. 
To determine whether the human enteric virome has homeostatic or pathophysiological properties, we purified virus-like particles (VLPs) from fresh colon resections taken from non-inflamed/nonIBD, Ulcerative Colitis (UC) or Crohn's disease (CD) patients, post-surgery (Fig 1A, Table S1). We reasoned that since viruses are obligate intracellular pathogens that rely on a host organism for successful replication, and bacteriophages reside within mucosal layers (5) and are readily taken up by eukaryotic cells $(27,30,31)$ certain viruses may be better represented in upper intestinal tissue than in feces. Tissue from non-IBD individuals was confirmed to be not inflamed and UC and $C D$ tissue was confirmed to be inflamed by a blinded clinical pathologist (Table S1). We quantified our VLP preparations by confocal microscopy using nucleic acid-binding SYBR Gold, and found an average of $2.8 \times 10^{7}$ VLPs per $\mathrm{mg}$ of colon tissue from non-IBD, UC or CD individuals (Fig 1B). Our preparations were comprised of prokaryotic and eukaryotic viruses, as visualized by electron microscopy (Fig S1A), and importantly, human colon-derived VLP preparations were both endotoxin- and cytokine-free (Fig S1B-F).

Our understanding of the host innate immune response to virus stems predominantly from studies of resolving acute infection. This mammalian antiviral paradigm centers on the detection of virus moieties by cytosolic or endosomal receptors and production of type I or III interferon (IFNs) and hundreds of IFN stimulated genes (ISGs) that aim to eliminate virus, or the cell that it infects (32). The rules governing if and how innate immune cells chronically respond to persistent eukaryotic or prokaryotic viruses in the intestine, as well as in other systemic niches (33), and how this shapes host physiology, are still poorly understood. Macrophages are one of the most abundant leukocytes in the intestinal mucosa where they are essential for maintaining homeostasis but are also implicated in the pathogenesis of IBD (34). Macrophages are also critical sensors of virus, including endocytosed bacteriophage (27). Therefore, to identify the precise innate immune response initiated by eukaryotic or prokaryotic viruses residing in healthy or diseased human colon, we exposed primary human peripheral blood derived macrophages to collective VLPs isolated from non-IBD, UC or CD patient colon resections (Fig 1A). Fluorescently labeled colon resection-derived VLPs demonstrated dose-dependent internalization by primary human macrophages that was equal for non-IBD, UC or CD-derived viromes (Fig 1C, D). Furthermore, virome uptake was dependent on endocytosis as treatment with brefeldin A a vesicular transport inhibitor, greatly reduced intracellular accumulation (Fig. S2). Thus, the human virome, that includes both eukaryotic viruses and bacteriophages, is readily internalized by human immune cells via endocytosis for pathogen associated molecular pattern (PAMP) recognition, consistent with other reports $(27,30)$. To assess the transcriptional pathways induced by the human colon tissue virome in macrophages, we performed unbiased RNA sequencing. Multi-dimensional scaling (MDS) analysis found non-IBD colon-resident viromes initiated a distinct transcriptional program, that was highly reproducible between 5 different patient VLP sources (Fig 1E). Conversely, UC or $\mathrm{CD}$ human enteric viromes from multiple patients induced macrophage transcriptional programs that were distinct from non-IBD virome-induced programs but almost indistinguishable from one another (Fig 1E). Interestingly, viruses resident to normal colon tissue predominantly suppressed the host innate immune response as more transcripts were down-regulated in macrophages exposed to non-IBD virus than were up-regulated (Fig S3). On the other hand, both UC and CD viromes triggered a prominent upregulation in gene expression of macrophages and these transcriptional programs were almost identical (Fig S3). A striking feature of the non-IBD virome induced macrophage transcriptional signature was a broad downregulation of the classical antiviral 
response. Non-IBD viromes significantly downregulated an array of virus receptors, signalling adapters and IFN stimuated genes (ISGs) such as TLR7, TLR8, IFIT2, IFIT3, RSAD2 (also known as Viperin) and IFI44L (Fig 1F and Fig S3D). This atypical host response to non-IBD viromes was also exemplified by the prominent induction of anti-inflammatory and pro-survival genes such as IL4R, CISH, BCL2, SOCS2 (Fig 1F) and multiple genes that define a homeostatic/resolving macrophage state $(35,36)$ (Fig S3C). In stark contrast, UC and CD viromes both robustly promoted inflammatory pathways in macrophages including many cytokines (IL6, ILIB, IL8, $I F N G$ ) and chemokines (CCL1, CCL20, CCL24, CXCL3, CXCL5, Fig 1G, H). Gene-set enrichment analysis defined the significant pathways downregulated by non-IBD viromes to be apoptosis, inflammatory and antiviral (Fig 1I) while those pathways that were upregulated were pro-survival and pathways known to polarize macrophages toward homeostatic/resolving states such as WNT- $\beta$ Catenin signaling (Fig 1I). In constrast, both UC and CD viromes robustly promoted inflammatory and apoptotic pathways (Fig 1J,K). We next confirmed the divergent macrophage activation states induced by non-IBD and IBD viromes using ELISA and qPCR with VLPs isolated from additional patient colon resections. Cytokines IL-10, IL-22, TGF $\beta$ or negative regulators of inflammation $C I S H$ or SOCS2, hallmark genes of anti-inflammatory and tissue remodeling type macrophages $(35,36)$ were all consistently and significantly elevated in macrophages exposed to VLPs isolated from 12 different non-IBD patient colon resections (Fig 1L, Fig S4). Notably, IL-22 induction is consistent with recent reports of mice mono-associated with apathogenic eukaryotic enteric viruses (37). Conversely, macrophages that internalized viromes derived from $\mathrm{UC}$ or CD intestinal resections from 8-12 separate patients, repeatedly displayed significantly enhanced production of cytokines TNF, IL-6, Type I and II IFNs, IL-1 $\beta$ and IL-12 that define pro-inflammatory type macrophages as well as other cytokines such as IL-15, IL-8 and IL-31 with designated roles in intestinal inflammation and IBD $(38,39)$ (Fig 1M, Fig S4). Importantly, inhibition of endocytosis or heat inactivation of VLPs, failed to induce macrophage cytokine responses (Fig S5). However, crosslinking of VLP preparations did not significantly reduce innate responses indicating that virus replication is not an absolute requirement for virome stimulatory capacity (Fig S5). Moreover, we observed similar divergent macrophage responses with virus isolated from non-IBD, UC and CD ileum lumen contents (Fig 1L,M, Fig S4, S6, Table S2). Taken together, these results demonstrate that human enteric viruses residing in colon tissue or ileum content autonomously invoke host innate immune responses. The macrophage response to enteric viruses is atypical to the classical antiviral response normally characterized by the induction of type I IFNs and ISGs. Enteric viruses from a healthy intestine instead dampened the classical antiviral response and promoted anti-inflammatory and prosurvival programs. Conversely, enteric viruses existing within an inflamed UC or CD intestine independently and robustly promoted a state of inflammation.

Intestinal epithelial cells (IECs) express innate immune receptors that recognize and respond to commensal microorganisms. Furthermore, bacteriophages are readily internalized by IECs (30, 31 ). We therefore also tested the ability of healthy or IBD enteric viruses to affect epithelial barrier function and the immune response of IECs. We utilized a trans-well Caco- 2 cell monolayer system and exposed IEC monolayers with viruses isolated from non-IBD, UC or CD colon resections and observed significantly more barrier integrity damage induced by both UC and CD-derived enteric viruses in a TLR4-independent manner, as measured by transepithelial electrical resistance (TEER, Fig S7A,B). Disrupted barrier integrity elicited by IBD viruses was also associated with a greater downregulation of transcripts for tight junction proteins Zonula occludens-1 (ZO-1) and Occludin 
(Fig S7C,D). Importantly, IFN $\lambda$ which controls both acute and persistent viral infections in the intestine $(40,41)$ and confers protection from intestinal inflammation (20) was preferentially induced by non-IBD viruses (Fig S7E). In contrast, viruses isolated from either UC or CD colons promoted production of pro-inflammatory cytokines such as TNF and IL-15 (Fig S7F,G) that play crucial roles in intestinal tissue destruction and the pathogenesis of IBD $(38,39,42)$. Of note, IL15 was also recently shown to be induced by the murine enteric virome in dendritic cells to promote expansion of innate epithelial lymphocytes (IELs) (23). Thus, similar to macrophages, IECs sense and divergently respond to non-IBD and IBD enteric viruses whereby IBD viromes promote both inflammatory responses and disruption of epithelial barrier function.

Fecal microbiota transplants (FMT) to restore disrupted microbiota are proving beneficial in both experimental and clinical settings (43) but the virome content of these preparations is currently not considered. We wondered if isolated healthy enteric viruses could overcome the inflammatory response induced by IBD viruses. To this end, we mixed increasing ratios of non-IBD colon resection derived viruses with IBD viruses while maintaining the same overall number of VLPs and exposed human macrophages to them. Increasing amounts of non-IBD viruses were capable of suppressing the ability of IBD-derived viruses to induce pro-inflammatory cytokines such as IL-6, TNF and IL-15 in a dose-dependent manner (Fig 1N, Fig S8A,B). Moreover, increasing the ratio of non-IBD viruses could partially restore the ability of macrophages to produce antiinflammatory cytokines such as IL-22 (Fig S8C). To test if the product of the immune response to healthy viruses in the form of secreted soluble factors could also suppress the IBD virome responses, we performed supernatant transfer experiments. In brief, human macrophages were exposed to non-IBD VLPs for 24 hours, then supernatant was collected, clarified, and placed on fresh cells in the presence of IBD colon-derived VLPs. Supernatant from non-IBD VLP treated macrophages was capable of suppressing the production of IL-6, TNF, IL-15 by IBD VLPs (Fig 10, Fig S8D, E). Furthermore, heat inactivation ameliorated suppressive activity of non-IBD virome-induced macrophage supernatant but UV crosslinking did not (Fig 10, Supp Fig 8D,E) demonstrating soluble proteins, but not residual viruses in the supernatant, were eliciting antiinflammatory activity. Thus, this data demonstrates the potential utility of viruses in a healthy intestine, or the anti-inflammatory immune response to it, to suppress the inflammatory capacity of viruses in a diseased intestine.

To gain a mechanistic understanding of the divergent immunomodulation by non-IBD or IBD derived human colon tissue or ileostomy fluid-derived viromes, we profiled the virus types in each cohort since only fecal VLPs have been examined to date. DNA and RNA were purified from preparations of VLPs from surgical colon resections or ileostomy fluid and characterized by metagenomic sequencing. After filtering out human and other contaminant (primers and adapters) and low-quality data, sequences were assigned a eukaryotic viral or bacteriophage taxonomic lineage. Specific eukaryotic viral families were selected for subsequent analysis based on the 40 alignment length and percent identity for each family that separated the high-quality alignments from potentially spurious alignments (Table S3). Total reads aligning to eukaryotic viruses were similar for non-IBD, UC or CD patients, however there was a significant increase in bacteriophage reads in $\mathrm{CD}$ patients (Fig $\mathbf{2 A}$ ). Of the high-quality reads for subsequent analysis, we found Papillomaviridae, Picornoviridae, Coronoviridae, Orthomyxoviridae and Anelloviridae animal 45 viruses, Virgiviridae plant viruses and a number of unknown eukaryotic virus families in colon tissue (Fig 2B). Furthermore, we found the majority of VLP classifications in human colon tissue 
to be bacteriophage families consisting of dsDNA Caudovirales order including Siphoviridae, Myoviridae, Podoviridae and unknown; dsDNA families Herelleviridae, Akkermanniviridae, Lipothrixviridae, as well as ssDNA phage family Microviridae. Quantification of viral species richness showed no significant difference between groups (Fig S9A) but differential analysis showed a significant elevation in the Picornoviridae family member Enterovirus B, in both UC and CD colon tissue compared to non-IBD controls (Fig 2C). Enterovirus $B$ has not been reported in fecal metagenomic studies performed to date, but has been previously detected in resected terminal ileum of immunocompetent pediatric patients with Crohn's disease (44). This further highlights the need to catalog viruses and other microorganisms (45) in intestinal tissue that is not fully captured in fecal material. Human enteroviruses are extremely common RNA viruses that spread mainly through the fecal-oral route. Species B enteroviruses consist of coxsackieviruses B1-B6 (CVB1-6), coxsackievirus A9 (CVA9), over 30 serotypes of echoviruses and more than $20 \mathrm{EV}-\mathrm{B}$ serotypes that all share the same overall structure found in picornaviruses, a single stranded $\sim 7500$ bp RNA genome. We searched for specific Enterovirus B serotypes in our data and found high identity mapping to Echovirus B75, Echovirus B5 and Echovirus 26, that were significantly elevated in both UC and CD colon resection samples. The wide prevalence and persistence of enteroviruses within humans has suggested a potential role for these viruses in complex human disease, including type I diabetes (46). Here we now identify Enterovirus B, and particularly Echovirus serotypes, as a potential pathogenic factor in UC and CD.

For bacteriophages, CD colon tissue exclusively demonstrated a significant expansion, consistent with reports of CD fecal viromes (1). We found significant elevations in total phage aligned reads (Fig 2A), a distinct separation of CD from non-IBD and UC by NMDS analysis (Fig S9B) and a multitude of significantly increased genus of Caudovirales members Siphoviridae, Myoviridae and Podoviridae (Fig 2D-F, Fig S9C). Interestingly, ileostomy fluid VLPs showed significantly reduced viral diversity compared to colon derived VLPs (Fig S10) underscoring tissue rather than luminal contents to be the abundant source of virus in the intestine. Detectable eukaryotic families in ileostomy fluid were limited to Annelloviridae, Papillomaviridae, Picornoviridae and Virgiviridae eukaryotic viruses and the bacteriophage families were Caudovirales family members Siphoviridae, Myoviridae, Podoviridae and unknown; dsDNA families Herelleviridae, Akkermanniviridae, Lipothrixviridae as well as ssDNA phage family Microviridae (Fig S10).

We next determined the virus receptor requirement for host responses to human enteric viruses. Virus-derived RNA structures are recognized by pattern recognition receptors (PRRs) such as cytosolic (RIG-I)-like receptors (RLRs), or endosomal Toll-like receptor (TLR)3, 7, 8 and viral DNA is mainly recognized by cyclic GMP-AMP (cGAMP) synthase (cGAS) or TLR9 (47). So far, endosomal TLR3, TLR7, TLR9 and cytosolic RIG-I have all been shown to be essential for sensing of the healthy murine virome $(19,23,28)$. We generated bone marrow-derived macrophages from mice deficient in the RLR signaling adapter mitochondrial antiviral signaling protein (MAVS), DNA sensor cGAS, TLR adapter protein MyD88 or endotoxin sensor TLR4, and exposed them to human colon VLPs (Fig 3A,B). Indeed, MAVS, cGAS or MyD88 deletion resulted in attenuated non-IBD virome responses, as measured by pro-survival gene Cish (Fig 3A). Conversely, deletion of endotoxin receptor TLR4 had little effect (Fig 3A), underscoring recognition of virus nucleic acid in VLP-driven responses. Similarly, pro-inflammatory cytokine IL-6 production by colon tissue derived IBD VLPs was also significantly diminished in MAVS-, cGAS-, and MyD88-deficient macrophages but was unchanged in TLR4-deficient macrophages 
(Fig. 3B). Notably, ileostomy fluid-derived VLPs, that had significantly reduced virus diversity compared to colon tissue (Fig S10) and was mostly bacteriophages and animal viruses of the Annelloviridae family (Fig S10), required DNA sensor cGAS exclusively for cytokine induction. Certainly, a dominance of viral-derived DNA was observed in the ileum content whilst the colonic tissue virome was a mix of RNA and DNA (Fig S11). This data suggests there is recognition redundancy for normal/non-IBD or IBD enteric viruses but qualitative and quantitative differences in the downstream immune responses induced by them. RLRs RIG-I (retinoic acid-inducible gene I; encoded by $D D X 58$ ) and MDA5 (melanoma differentiation-associated gene 5; encoded by IFIH1) are activated by distinct viral RNA structures to differentiate between viral and cellular RNAs. MDA5 senses long double-stranded (ds)RNA, while RIG-I responds to blunt-ended dsRNA bearing a triphosphate (ppp) moiety (48). Transfection of purified RNA isolated from nonIBD VLPs, triggered more IL-10 than equal concentrations of RNA from UC or CD VLPs (Fig 3C). In contrast, UC or CD VLP RNA triggered more IL-6 production (Fig 3D) demonstrating divergent macrophage responses were not due to differences in viral tropism or levels. Importantly, digestion of single-stranded or double-stranded VLP RNA with RNase I or III, respectively, or removal of phosphates with Calf Intestinal Phosphatase (CIP), eliminated this response (Fig 3C, D) demonstrating the existence of "non-self" RNA moieties within the human enteric virome and the requirement for both RIG-I and MDA5 in their recognition. Divergent anti- or proinflammatory responses by non-IBD or IBD enteric viruses were also induced by transfection of VLP-derived DNA, in a cGAS-dependent manner (Fig 3E, F). Thus, altered RNA or DNA moieties that are sensed by host virus receptors RIG-I, MDA-5 or cGAS (and likely other receptors), rather than an adaptive function of viral proteins on the host, contribute to the divergent activation thresholds and immunomodulation by normal/non-IBD and IBD enteric viromes. This deviation in RNA and DNA viral ligands within the diseased virome is attributed to the fluctuations of certain viruses we observed (Fig 2). However, it is also appealing to speculate that akin to some bacteria (49), normally symbiotic viruses may also become pathobionts when in an inflamed environment.

Given the existence of genetic mutations within MDA5 that were identified to associate with IBD incidence by GWAS (29) and the discovery of elevated Enterovirus B of the Picornoviridae family in IBD colon tissue, that is exclusively sensed by MDA5 (50), we next examined the consequence of these mutations on responses to the human non-IBD and IBD enteric virome. IFIHI gain-offunction has been reported as a cause of a type I interferonopathy encompassing a spectrum of autoinflammatory phenotypes including Systemic Lupus Erythematosus and Aicardi-Goutières syndrome (51). Yet, intriguingly, all IBD-associated IFIH1 variants have predicted loss-offunction biological effects, either truncating the protein (rs35744605, E627X), affecting essential splicing positions (rs35732034 in a conserved splice donor site at position +1 in intron 14) or altering a highly conserved amino acid (rs35667974/I923V; Fig 3G) within the C-terminal domain (CTD) which recognizes and binds to RNA (52). We transfected HEK-293T cells with wild-type MDA5 or E627X or I923V MDA5 coding variants and found the wild-type and MDA5 mutants were expressed at comparable levels although the E627X truncation, that lacks a part of the helicase domain and the entire CTD in which 1923 resides, showed slightly reduced MDA5 protein levels (Fig S12). Wild-type MDA5 conferred similar responsiveness to the synthetic RNA ligand poly(I:C) and RNA isolated from healthy human colon tissue VLPs but was not responsive to self RNA (Fig S12). However, both IBD-associated MDA5 variants exhibited a loss of function phenotype to poly(I:C) (Fig S12) in agreement with a previous report (53). Importantly, a loss of 
function phenotype to human colon derived viral RNA was observed for both IBD-associated coding MDA5 variants (Fig S12). We next examined the functional consequence of a loss of MDA5 function on intestinal health. MDA5 is predominantly expressed in epithelial cells (Fig S13) so we examined functional consequences of loss-of-function MDA5 by converting induced pluripotent stem cells (iPSCs) derived from a compound heterozygote UC patient carrying a single allele of rs35732034/IVS14+1 and a single allele of rs35744605/E627X into human intestinal epithelial cells (hIECs, Fig 3H, Fig S14). Compared to non-affected individuals, hIECs from patients carrying loss-of-function MDA $5^{\mathrm{E} 627 \mathrm{X} / \mathrm{IVS} 14+1}$ displayed similar morphology and barrier integrity at steady state, as measured by confocal microscopy for F-actin, ZO1 and E-cadherin (Fig 3I). However, unlike iPSC-derived hIECs cells bearing wild-type MDA5, MDA5 ${ }^{\mathrm{E} 627 \mathrm{X} / \mathrm{IV} 14+1}$ iPSC-derived hIECs were severely impaired in their ability to maintain barrier integrity when exposed to enteric viruses derived from non-IBD colon resections (Fig 3J). Furthermore, damage to MDA5 $5^{\text {E627X/IVS14+1 }}$ hIECs was exacerbated when exposed to IBD derived viromes containing

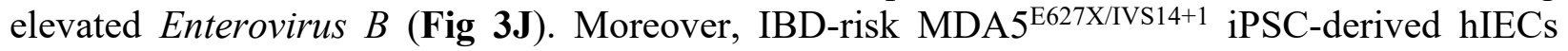
displayed significantly abrogated virome-induced IFN $\lambda$ production compared to wild-type cells (Fig 3K). Thus, abolished innate responses to the virome due to human genetic variation within virus receptor MDA5 has detrimental consequences for intestinal epithelial cell function and integrity. Moreover, intestinal damage is intensified in patients bearing IBD-risk MDA5 variants responding to an IBD virome, underscoring the ability of environmental cues to uniquely impact IBD phenotypes in the context of host genetics.

We next assessed the ability of non-IBD or IBD human colon-resident viruses to shape local immunity and intestinal disease in vivo. To achieve this, we first diminished the murine enteric virome of C57BL/6 using an antiviral (AV) cocktail by oral gavage for 10 days (19) and then administered human colon resection-derived VLPs 3 times intragastrically (Fig 4A). To complement our functional data in macrophages, we first assessed the influence of healthy or diseased enteric viromes on gut resident mononuclear phagocytes (MNPs), especially those bearing the fractalkine receptor CX3CR1 (CX3CR1 $\left.1^{+} \mathrm{MNPs}\right)$, since they have established roles in the induction of immune responses toward enteric bacteria (54) and more recently intestinal fungi (55). In parallel, we examined the influence of human enteric viromes on subsets of dendritic cells (DCs) differentially expressing integrins CD11b and CD103 that initiate immune tolerance (56, 57). Mice with a healthy humanized virome showed no changes in lamina propria CX $3 \mathrm{CR} 1^{+} \mathrm{MNPs}$ compared to wild-type or antiviral treated mice (Fig 4B,C) but had elevated tolerizing CD $11 b^{+}$CD $11 c^{+}$phagocytes expressing CD103 (Fig 4B,C, Fig S15). In contrast, mice with an IBD humanized virome displayed a significant accumulation of MNPs expressing CX3CR1 and a

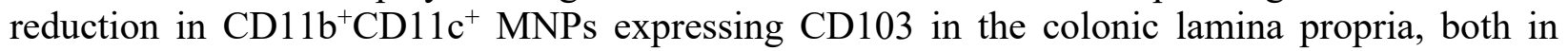
number and as a fraction of the total $\mathrm{CD}_{11} \mathrm{~b}^{+}$phagocyte compartment (Fig 4B,C, Fig S15). Thus, IBD-associated enteric viruses can promote an inflammatory state in the intestine in vivo by spontaneously influencing the ratio of $\mathrm{CX} 3 \mathrm{CR} 1^{+}$and $\mathrm{CD}_{103}{ }^{+}$lamina propria MNPs.

The ability of the human enteric virome to contribute to disease phenotype in a dextran sulfate sodium (DSS)-induced colitis model was then assessed. Since this model is more closely associated with UC, mice diminished of their murine virome were administered human enteric viruses derived from colon resections of non-IBD or UC or CD patients 3 times intragastrically 45 (Fig 4D). We assessed the abundance of isolated virus-like particles (VLPs) in each treatment group by staining fecal or colon tissue derived VLPs with SYBR. We found a mean of $1.1 \times 10^{7}$ 
VLPs/mg of feces and $1.1 \times 10^{6} \mathrm{VLPs} / \mathrm{mg}$ of colon tissue in naïve C57BL/6 mice (Fig 4E,F). After AV cocktail treatment, fecal VLPs declined $\sim 20$-fold to a mean of $6 \times 10^{5} \mathrm{VLPs} / \mathrm{mg}$ in accordance with previous publications in Balb/c mice (19) and VLPs in colon tissue declined $\sim 12$ fold to $9 \times 10^{4}$ $\mathrm{VLP} / \mathrm{mg}$. Quantification of viruses in feces or colon tissue of humanized virome mice revealed an equal abundance of $\sim 4 \times 10^{6} \mathrm{VLPs} / \mathrm{mg}$ of feces and $\sim 4 \times 10^{5} \mathrm{VLPs} / \mathrm{mg}$ of colon tissue from mice humanized with healthy, UC or CD viromes (Fig 4E, F). Consistent with recent publications (19, 20), depletion of viruses in healthy mice lead to significantly exacerbated DSS induced intestinal damage (Fig 4G-I). Remarkably, mice repopulated with a healthy humanized virome conferred robust protection against colitis, including less shortening of the colon (Fig. 4G), rescued stool lipocalin levels (Fig. 4H), rescued colon IL-6 levels (Fig 4I). Thus, normal/non-IBD human enteric viromes actively immunodulate and incite protection from intestinal inflammation in vivo, consistent with our in vitro results. In contrast, both UC or CD human colonic viromes were incapable of rescuing antiviral treated mice and promoted intestinal disease in vivo (Fig 4G-I), results that were confirmed by histological analysis (Fig 4J,K). Moreover, confirming our in vitro data, both UC or CD intestinal viromes resulted in reduced intestinal barrier integrity in vivo, as measured by gavaged FITC-dextran in the serum (Fig 4L). We had demonstrated an autonomous immunodulatory capacity of intestine-derived viromes in cells, however, to assess any viromemicrobiome community interactions vivo, we performed $16 \mathrm{~S}$ sequencing of host microbiota. Bacterial diversity and richness were not significantly affected by antiviral treatment or gavage administration of human healthy or IBD viromes (Fig S16A). However, in UniFrac-based PCoA analysis, groups were clustered separately dependent on virus depletion or human virome source $(\mathrm{P}<0.05$, PERMANOVA, Fig S16B). By proportion (Fig S16C) and LEfSe analysis, the bacterial groups most changed by AV cocktail treatment was a reduction in the phyla Firmicutes and an elevation certain genera of the Ruminococcaceae family (Fig S16D). Both healthy and UC humanized virome mice displayed similar changes in murine microbiota (Fig S16E) suggesting microbiome changes are unlikely to be the driver of divergent human virome-induced phenotypes. Furthermore, CD humanized virome mice had unique changes in the murine microbiome (Fig S16F) reflecting the distinct bacteriophage alterations we had observed in CD colon tissue. Importantly however, virus nucleic acid sensing by the host immune system was an absolute requirement for immunomodulation by healthy or IBD human viromes in vivo. Mice deficient in MAVS, the adapter protein of RNA sensors RIG-I and MDA5, or mice deficient in the DNA sensor cGAS, displayed no change in colon length, lipocalin or colon IL-6 production following administration of non-IBD, UC or CD human enteric viromes, compared to antiviral-treated controls (Fig 4M-R). Thus, an ability of human enteric viromes to directly shape intestinal disease phenotype in vivo was via host innate immune recognition of viruses. Moreover, it demonstrates that fluctuations in the virome are not correlative with disease but rather there are direct functional consequences on host immune and intestinal state due to active host sensing of an altered virome in $\operatorname{IBD}(1,8,10)($ Fig 2)

While disruption of microbiome and virome has been observed in IBD and other diseases, a functional role for the virome has remained elusive. Our data demonstrate that viruses resident to normal human colon tissue are readily endocytosed and elicit an immunomodulatory transcriptional program in macrophages and intestinal epithelial cells, consistently promote production of anti-inflammatory cytokines, and can maintain homeostasis and protect against induced colitis in vivo. Conversely, viromes from inflamed intestines, and specifically UC and CD, triggered pro-inflammatory macrophage and IEC barrier loss phenotypes, and intestinal 
inflammation in vivo upon transfer. These functional differences were dependent on innate viral sensors, indicating active and unique recognition of the intestinal virome by the host. Altogether, our findings provide evidence that viruses in a normal/non-inflamed intestine can help build gut immunity in humans - a contrast to the misconception that all viruses are harmful. An alteration in the intestine-resident virome, or disrupted virome sensing due to genetic variation in the virus receptor MDA5, has detrimental consequences for the human intestine. It is appealing to speculate that viruses play a role in the protection mediated by fecal transplants, as well as in the pathology of intestinal inflammation. Therapeutic modulation of the virome through targeted elimination or replacement of disease- or health-driving intestinal viruses presents an novel strategy to treat intestinal and immunological diseases more broadly.

15 1. J. M. Norman et al., Disease-specific alterations in the enteric virome in inflammatory bowel disease. Cell 160, 447-460 (2015).

2. G. Liang et al., The stepwise assembly of the neonatal virome is modulated by breastfeeding. Nature 581, 470-474 (2020).

3. R. A. Edwards et al., Global phylogeography and ancient evolution of the widespread human gut virus crAssphage. Nat Microbiol 4, 1727-1736 (2019).

4. L. F. Camarillo-Guerrero, A. Almeida, G. Rangel-Pineros, R. D. Finn, T. D. Lawley, Massive expansion of human gut bacteriophage diversity. Cell 184, 1098-1109 e1099 (2021).

5. J. J. Barr et al., Bacteriophage adhering to mucus provide a non-host-derived immunity.

6. E. S. Lim et al., Early life dynamics of the human gut virome and bacterial microbiome in infants. Nat Med 21, 1228-1234 (2015).

7. F. Adiliaghdam, K. L. Jeffrey, Illuminating the human virome in health and disease. Genome Med 12, 66 (2020).

30 8. A. G. Clooney et al., Whole-Virome Analysis Sheds Light on Viral Dark Matter in Inflammatory Bowel Disease. Cell Host Microbe, (2019).

9. B. A. Duerkop et al., Murine colitis reveals a disease-associated bacteriophage community. Nat Microbiol 3, 1023-1031 (2018).

10. G. Liang et al., The dynamics of the stool virome in very early onset inflammatory bowel

11. G. D. Hannigan, M. B. Duhaime, M. T. t. Ruffin, C. C. Koumpouras, P. D. Schloss, Diagnostic Potential and Interactive Dynamics of the Colorectal Cancer Virome. MBio 9, (2018).

12. G. Nakatsu et al., Alterations in Enteric Virome Are Associated With Colorectal Cancer and Survival Outcomes. Gastroenterology 155, 529-541 e525 (2018).

13. G. Zhao et al., Intestinal virome changes precede autoimmunity in type I diabetessusceptible children. Proc Natl Acad Sci U S A 114, E6166-E6175 (2017).

14. S. Lang et al., Intestinal Virome Signature Associated With Severity of Nonalcoholic Fatty Liver Disease. Gastroenterology, (2020). 
15. D. Willner et al., Metagenomic analysis of respiratory tract DNA viral communities in cystic fibrosis and non-cystic fibrosis individuals. PLoS One 4, e7370 (2009).

16. J. Legoff et al., The eukaryotic gut virome in hematopoietic stem cell transplantation: new clues in enteric graft-versus-host disease. Nat Med 23, 1080-1085 (2017).

17. C. L. Monaco et al., Altered Virome and Bacterial Microbiome in Human Immunodeficiency Virus-Associated Acquired Immunodeficiency Syndrome. Cell Host Microbe 19, 311-322 (2016).

18. I. D. Iliev, K. Cadwell, Effects of Intestinal Fungi and Viruses on Immune Responses and Inflammatory Bowel Diseases. Gastroenterology, (2020).

19. J. Y. Yang et al., Enteric Viruses Ameliorate Gut Inflammation via Toll-like Receptor 3 and Toll-like Receptor 7-Mediated Interferon-beta Production. Immunity 44, 889-900 (2016).

20. A. Broggi, Y. Tan, F. Granucci, I. Zanoni, IFN-lambda suppresses intestinal inflammation by non-translational regulation of neutrophil function. Nat Immunol 18, 1084-1093 (2017).

21. A. E. Price et al., A Map of Toll-like Receptor Expression in the Intestinal Epithelium Reveals Distinct Spatial, Cell Type-Specific, and Temporal Patterns. Immunity 49, 560575 e566 (2018).

22. Y. Wang et al., Rig-I-/- mice develop colitis associated with downregulation of G alpha i2. Cell Res 17, 858-868 (2007).

20 23. L. Liu et al., Commensal viruses maintain intestinal intraepithelial lymphocytes via noncanonical RIG-I signaling. Nat Immunol 20, 1681-1691 (2019).

24. E. Kernbauer, Y. Ding, K. Cadwell, An enteric virus can replace the beneficial function of commensal bacteria. Nature 516, 94-98 (2014).

25. J. A. Neil et al., IFN-I and IL-22 mediate protective effects of intestinal viral infection. Nat Microbiol, (2019).

26. X. D. Li et al., Mitochondrial antiviral signaling protein (MAVS) monitors commensal bacteria and induces an immune response that prevents experimental colitis. Proc Natl Acad Sci U S A 108, 17390-17395 (2011).

27. J. M. Sweere et al., Bacteriophage trigger antiviral immunity and prevent clearance of bacterial infection. Science 363, (2019).

28. L. Gogokhia et al., Expansion of Bacteriophages Is Linked to Aggravated Intestinal Inflammation and Colitis. Cell Host Microbe 25, 285-299 e288 (2019).

29. H. Huang et al., Fine-mapping inflammatory bowel disease loci to single-variant resolution. Nature 547, 173-178 (2017).

35 30. S. Nguyen et al., Bacteriophage Transcytosis Provides a Mechanism To Cross Epithelial Cell Layers. mBio 8, (2017).

31. M. C. Bichet et al., Bacteriophage uptake by mammalian cell layers represents a potential sink that may impact phage therapy. iScience 24, 102287 (2021).

32. A. Park, A. Iwasaki, Type I and Type III Interferons - Induction, Signaling, Evasion, and Application to Combat COVID-19. Cell Host Microbe 27, 870-878 (2020).

33. H. W. Virgin, The virome in mammalian physiology and disease. Cell 157, 142-150 (2014).

34. E. Zigmond, S. Jung, Intestinal macrophages: well educated exceptions from the rule. Trends Immunol 34, 162-168 (2013).

45 35. P. J. Murray et al., Macrophage activation and polarization: nomenclature and experimental guidelines. Immunity 41, 14-20 (2014). 
36. F. O. Martinez et al., Genetic programs expressed in resting and IL-4 alternatively activated mouse and human macrophages: similarities and differences. Blood 121, e57-69 (2013).

37. S. Dallari et al., Enteric viruses evoke broad host immune responses resembling those elicited by the bacterial microbiome. Cell Host Microbe, (2021).

38. B. Jabri, V. Abadie, IL-15 functions as a danger signal to regulate tissue-resident T cells and tissue destruction. Nat Rev Immunol 15, 771-783 (2015).

39. M. F. Neurath, Cytokines in inflammatory bowel disease. Nat Rev Immunol 14, 329-342 (2014).

40. J. Pott et al., IFN-lambda determines the intestinal epithelial antiviral host defense. Proc Natl Acad Sci U S A 108, 7944-7949 (2011).

41. M. T. Baldridge et al., Commensal microbes and interferon-lambda determine persistence of enteric murine norovirus infection. Science 347, 266-269 (2015).

42. D. B. Graham, R. J. Xavier, Pathway paradigms revealed from the genetics of inflammatory bowel disease. Nature 578, 527-539 (2020).

43. E. M. Giles, G. L. D'Adamo, S. C. Forster, The future of faecal transplants. Nat Rev Microbiol 17, 719 (2019).

44. N. Nystrom et al., Human enterovirus species B in ileocecal Crohn's disease. Clin Transl Gastroenterol 4, e38 (2013).

45. U. Jain et al., Debaryomyces is enriched in Crohn's disease intestinal tissue and impairs

46. D. Hober, P. Sauter, Pathogenesis of type 1 diabetes mellitus: interplay between enterovirus and host. Nat Rev Endocrinol 6, 279-289 (2010).

47. X. Tan, L. Sun, J. Chen, Z. J. Chen, Detection of Microbial Infections Through Innate Immune Sensing of Nucleic Acids. Annu Rev Microbiol 72, 447-478 (2018).

48. D. Goubau, S. Deddouche, C. Reis e Sousa, Cytosolic sensing of viruses. Immunity 38, 855-869 (2013).

49. L. Jochum, B. Stecher, Label or Concept - What Is a Pathobiont? Trends Microbiol, (2020).

50. H. Kato et al., Differential roles of MDA5 and RIG-I helicases in the recognition of RNA viruses. Nature 441, 101-105 (2006).

51. G. I. Rice et al., Genetic and phenotypic spectrum associated with IFIH1 gain-of-function. Hum Mutat 41, 837-849 (2020).

52. A. Ablasser, S. Hur, Regulation of cGAS- and RLR-mediated immunity to nucleic acids. Nat Immunol 21, 17-29 (2020).

53. T. Shigemoto et al., Identification of loss of function mutations in human genes encoding RIG-I and MDA5: implications for resistance to type I diabetes. J Biol Chem 284, 1334813354 (2009).

54. M. Kim et al., Critical Role for the Microbiota in CX3CR1(+) Intestinal Mononuclear Phagocyte Regulation of Intestinal T Cell Responses. Immunity 49, 151-163 e155 (2018).

40 55. I. Leonardi et al., CX3CR1 $(+)$ mononuclear phagocytes control immunity to intestinal fungi. Science 359, 232-236 (2018).

56. J. L. Coombes et al., A functionally specialized population of mucosal CD103+ DCs induces Foxp3+ regulatory T cells via a TGF-beta and retinoic acid-dependent mechanism. $J$ Exp Med 204, 1757-1764 (2007). 
57. M. A. Kinnebrew et al., Interleukin 23 production by intestinal CD $103(+) C D 11 b(+)$ dendritic cells in response to bacterial flagellin enhances mucosal innate immune defense. Immunity 36, 276-287 (2012).

Acknowledgments: The authors wish to thank the clinical coordinators and patients enrolled in the Prospective Registry in IBD study at Massachusetts General Hospital (PRISM). We would also like to thank the MGH Next Gen Sequencing core, Thomas Diefenbach at the Ragon Institute Imaging Core for confocal imaging of VLPs, the Harvard Medical School electron microscopy facility, Hembly Rivas (Harvard Virology Ph.D. program) for assistance with MDA5 experiments, Laurie Pruneau and Marie-Eve Rivard (Montreal Heart Institute) for technical expertise with iPSC to human epithelial cell trans-differentiation using LCL cells lines from NIDDK Inflammatory Bowel Disease Genetics Consortium (IBDGC) and Chloé Lévesque for confocal immunofluorescence analyses of the hiPSC-derived epithelial 2D cultures. The NIDDK IBDGC was conducted by the IBDGC Investigators and supported by the National Institute of Diabetes and Digestive and Kidney Diseases (NIDDK). The lymphoblastoid cell lines and related data from the IBDGC reported here were supplied by the NIDDK Central Repositories. Other than J.D.R., this manuscript was not prepared in collaboration with investigators of the IBDGC study and does not necessarily reflect the opinions or views of the IBDGC study, the NIDDK Central Repositories, or the NIDDK. We thank Amy Avery (Massachusetts General Hospital) for technical assistance with 16S sequencing and Raza Hoda (Massachusetts General Hospital) for blindly scoring intestinal pathology. We thank Corinne Maurice (McGill University), Irah King (McGill University), Naama Geeva-Zatorsky (Technion) and the entire Jeffrey lab for valuable scientific discussions and Robert Anthony, James Moon and Ramnik Xavier for critical reading of the manuscript.

Funding: This study was supported by the Kenneth Rainin Foundation (Innovator and Synergy Awards to K.L.J), NIH R21AI144877 (K.L.J), NIH R01DK119996 (K.L.J), Harvard Catalyst | The Harvard Clinical and Translational Science Center (National Center for Advancing Translational Sciences, National Institutes of Health Award UL 1TR002541) and financial contributions from Harvard University and its affiliated academic healthcare centers (K.L.J), Canadian Institute of Health Research (CIHR) postdoctoral fellowship (H.A.), NIH P30DK040561 (R.S.), Canada Research Chair (J.D.R), NIH DK062432 (J.D.R) and NIH RC2DK116713 (S.H.). K.L.J is a John Lawrence MGH Research Scholar 2020-2025.

Author contributions: F.A. conducted and analyzed the majority of in vitro and in vivo experiments, functional assays, developed methods, and generated resources; H.A. assisted with and analyzed VLP uptake flow cytometry and assisted with DSS models, S.D. performed RNA sequencing experiments and performed and analyzed lamina propria flow cytometry experiments with help from H.A.; T.S. assisted with in vivo and in vitro assays, L-P.W and R.S. analyzed RNA sequencing data; R-U.R. analyzed $16 \mathrm{~S}$ sequencing; J.P., P.G and J.D.R. provided lymphoblastoid cell lines (LCL) from MDA5 variant IBD patients and performed LCL to iPSC to intestinal epithelial trans-differentiation of these cells., R.H. provided clinical intestinal and ileostomy 
samples post-surgery; K.M and S.H. analyzed VLP sequencing; K.L.J conceived and supervised the study, acquired funding and wrote the final manuscript.

Competing interests: The authors declare no competing interests.

\section{Additional information}

\section{Author Information}

Correspondence and requests for materials should be addressed to Kate L. Jeffrey KJeffrey@mgh.harvard.edu

Data and materials availability: The data presented in this study are tabulated in the main text and supplementary materials. RNA sequencing raw and processed data has been deposited at Gene Expression Omnibus (GEO) and is accessible using accession number GSE135223. Human colon resection and ileostomy fluid virus like particle (VLP) sequencing are deposited at European Nucleotide Archive (ENA) under accession number PRJEB44121 and 16S rRNA of murine feces of humanized virome mice are deposited at Sequencing Read Archive (SRA) under accession numbers SUB9443038.

\section{Figure Legends}

Fig 1. Human colon tissue resident viruses protect while IBD viromes divergently provoke inflammation. (A) Virus-like particles (VLPs) isolated from colon resections post-surgery from non-IBD $(n=5)$, Ulcerative Colitis $(U C, n=5)$ or Crohn's disease $(C D, n=5)$ patients. (B) Visualization and quantification of VLP isolates per mg of colon tissue using confocal microscopy for RNA/DNA using SYBR Gold. Scale bar is $1 \mu \mathrm{m}$. (C, D) Flow cytometric quantification of uptake of SYTO-16 labeled human colon-resection derived VLPs by human peripheral bloodderived macrophages ( 1 or 10 VLPs per cell). Adsorption control is fluorescently labeled VLPs delivered to human macrophages at $4{ }^{\circ} \mathrm{C}$. (E) Multidimensional scaling (MDS) plot of human macrophage transcriptional profiles induced by non-IBD or UC or CD derived VLPs (10 VLPs/cell, 24h). (F-H) Volcano plots showing differentially expressed genes comparing F) nonIBD delivery to mock adsorption, (G) UC delivery to mock adsorption or (H) CD delivery to mock adsorption. (I-K) Hallmark gene sets significantly (P value $<0.05$, FDR $<0.20$ ) represented in (I) non-IBD, (J) UC or, (K) CD VLP induced transcriptional programs as assessed by Gene Set Enrichment Analysis (GSEA). NES is normalized enrichment score. (L) Production of antiinflammatory mediators Interleukin (IL)-10, IL-22 or CISH or (M) pro-inflammatory mediators TNF, IL-6, IL-15 as measured by ELISA or qPCR following delivery of VLPs (10 VLPs/cell, 24h) extracted from non-IBD, Ulcerative Colitis (UC) or Crohn's disease (CD) colon resections ( $\mathrm{n}=8$ per group) or ileostomy content ( $\mathrm{n}=8$ per group) to primary human peripheral blood-derived macrophages. (N) Primary human peripheral blood-derived macrophages were delivered colon resection derived non-IBD VLPs (10 VLPs/cell), IBD VLPs (10 VLPs/cell) or indicated ratios of non IBD to IBD VLPs (total of 10 VLPs/cell) for 24 hours and IL-6 was measured by ELISA. (O) IL-6 levels measured by ELISA following IBD VLP delivery to human macrophages in the presence of non-IBD macrophage supernatant that had been heat inactivated $\left(95^{\circ} \mathrm{C} 10 \mathrm{~min}\right)$ or UV crosslinked $\left(3 \mathrm{x} 200 \mathrm{~mJ} / \mathrm{cm}^{2}\right)$ as controls. Data are mean \pm s.e.m. of $8-12$ biological replicates 
$* P<0.05, * * P<0.01, * * * P<0.001, * * * * P<0.0001$ as determined by one-way ANOVA with Tukey's multiple comparison test.

Fig 2. Detection and characterization of VLPs in colon resections from non-IBD, Ulcerative Colitis and Crohn's disease patients. VLPs were assessed using VLP DNA and RNA metagenomic sequence data. Sequences were assigned a eukaryotic viral or phage taxonomic lineage using primary searches against virus sequence databases and subsequently confirmed in secondary searches using reference databases with additional non-viral taxonomic lineages. Specific eukaryotic viral families were selected for subsequent analysis by gating on the alignment length and percent identity for each family that separated the high-quality alignments from potentially spurious alignments (Table S3). (A) Violin plots represent the distribution of individual datasets for total reads aligning to eukaryotic viruses or bacteriophages from non-IBD, UC or CD cohorts; samples were compared using two-sided Wilcoxon signed-rank tests. (B) Taxonomic assignments of VLP sequences in non-IBD, UC or CD colon tissue. (C) Significantly elevated abundance of eukaryotic virus, Picornoviridae $>$ Enterovirus $B$ in UC and CD colon resections. (D) Identification of Echovirus B75, Echovirus E5 and Echovirus E26 Enterovirus B serotypes in UC or CD colon tissue by bbmap. All potential Picornavirales reads were re-mapped one at a time to six Enterovirus B genomes (KF874627.1, NC_001472.1, AF083069.1, AY302539.1, D00627.1, NC_038307.1), using the bbmap tool, mapPacBio.sh with the parameters recommended for remote homologies (vslow $\mathrm{k}=8$ maxindel $=200$ minratio $=0.1$ ). The subset of these reads that mapped to Enterovirus B, along with the previously identified Enterovirus B reads, were plotted, split into Serotypes on the X-axis and percent identity on the y-axis. Each read found is plotted and grouped and colored by the type of sample in which it was found (Non-IBD, UC or CD). Non-IBD versus UC and Non-IBD versus CD were significantly different ( $\mathrm{p}$-adj $=0.029$, 0.001). (E) Top significantly increased genera of Siphoviridae, (F) Myoviridae or (G) Podoviridae bacteriophages in CD colon resections. Data are the mean of 5-7 biologial replicates. $* P<0.05$, Kruskal-Wallis test with a Dunn's post-hoc test.

Fig 3. Host viral receptor requirement for sensing human enteric viruses and detrimental consequences for IBD patients with MDA5 loss-of-function variants. (A) Cish or (B) IL-6 levels following exposure of primary bone marrow derived macrophages (BMDMs) from wildtype, $\mathrm{MAVS}^{-/}$, $\mathrm{cGAS}^{-/-}, \mathrm{MyD}^{-/-}$or TLR4 ${ }^{-/}$mice to non-IBD, Ulcerative Colitis (UC) or Crohn's disease (CD) colon resection-derived VLPs as measured by qPCR and ELISA. (C) IL-10 or (D) IL-6 production $24 \mathrm{~h}$ following transfection of THP1 cells with $2.5 \mu \mathrm{g}$ of VLP RNA or VLP RNA pre-treated with RNase I $(5 \mu \mathrm{g} / \mathrm{mL}, 1 \mathrm{~h})$, RNase III $(5 \mu \mathrm{g} / \mathrm{mL}, 1 \mathrm{~h})$ or Calf Intestinal Phosphatase (CIP, 250U, 1h). (E) Cish levels or (F) IL-6 production $24 \mathrm{~h}$ following transfection of WT or cGAS/- BMDMs with $2.5 \mu \mathrm{g}$ of VLP DNA as measured by qPCR or ELISA. (G) Schematic of human MDA5 and mapped IBD-associated MDA5 variants rs35744605/E627X, rs35667974/I923V and rs35732034/IVS14+1. (H) Schematic of patient lymphoblastoid cell line (LBL) derived human induced pluripotent stem cells (hiPSCs) trans-differentiated into intestinal epithelial cell monolayers. (I) Differentiated mature monolayers evaluated for structural integrity markers. Upper panel, orthogonal view of F-actin (Phalloidin, red), tight junction protein ZO1 (green) and nuclei (DAPI, blue) by confocal microscopy. Scale bar represents $50 \mu \mathrm{m}$. Lower panel, maximal intensity projection images of epithelial adherens junction marker E-cadherin (gray) and nuclei (DAPI, blue). Scale bar is $20 \mu \mathrm{m}$. (J) Trans-epithelial electrical resistance (TEER) over indicated time or (K) IFNL 1 levels at 24h as measured by qPCR of WT or MDA5 E627Ter/IVS14+1 patient 
hiPSC-derived intestinal epithelial cell monolayers and exposed to VLPs (10 VLPs/cell) extracted from non-IBD or IBD colon resections. Data are mean \pm s.e.m. of 2-5 biological replicates repeated twice. $* P<0.05, * * P<0.01, * * * P<0.001, * * * * P<0.0001$ as determined by one-way ANOVA with Tukey's multiple comparison test.

Fig 4. Mice with a "humanized" non-IBD virome had attenuated intestinal inflammation while those with a "humanized" IBD-derived virome exhibited intestinal inflammation. (A) Schematic of mouse gut virome depletion using an antiviral cocktail (Acyclovir 20mg/kg, Lamivudine $10 \mathrm{mg} / \mathrm{kg}$, Ribavirin 30mg/kg, Oseltamivir $10 \mathrm{mg} / \mathrm{kg}$, gray, daily gavage for 10 days), and reconstitution with $200 \mu \mathrm{L}$ of human non-IBD or IBD VLPs pool ( $4 \times 10^{8}$ VLPs per mouse) by gavage on day 10, 12 and 14. 2.5\% DSS colitis model was commenced on day 16. (B) Flow cytometry plots of $\mathrm{CD}^{2} 5^{+} \mathrm{MHCII}^{+} \mathrm{CD} 11 \mathrm{c}^{+} \mathrm{CD} 11 \mathrm{~b}^{+}$cells from the colonic lamina propria, and percentages and counts of $(\mathbf{C}) \mathrm{CX} 3 \mathrm{CR} 1^{+}$or $\mathrm{CD} 103^{+}$mononuclear phagocytes in control, antiviral depleted (AV), non-IBD or IBD humanized virome mice on day 17. (D-F) Confocal images and quantification of Viral Like Particles (VLPs) using SYBR Gold in feces or colon tissue of humanized virome mice. (G) Colon length, (H) stool lipocalin, (I) IL-6 levels in colonic explant supernatants cultured for 24 hours following 12 days of induced DSS colitis measured by ELISA. $(\mathbf{J}, \mathbf{K})$ Representative hematoxylin and eosin-stained sections and blinded histologic scores in mouse colon tissue (scale bar, $50 \mu \mathrm{m}$ ). (L) FITC-dextran level in serum 4 hours after oral gavage following 7 days of DSS. (M-O) Colon length, stool lipocalin or colonic explant IL-6 levels following induction of DSS colitis in antiviral-treated MAVS-/- mice or (P-R) cGAS-/- mice administered human non-IBD, UC or CD colon-derived viromes. Data are mean \pm s.e.m. of 4-8 animals. Representative of 2 independent experiments. $* P<0.05, * * P<0.01, * * * P<0.001$, $* * * * P<0.0001$, two-tailed unpaired t test or one-way ANOVA with Tukey's multiple comparison test. 
A bioRxiv preprint doi: https://doi.org/10.1101/20 R 10.14 .464404 ; this version posted Ogtober 20 , 2021. The copyright holder for this preprint

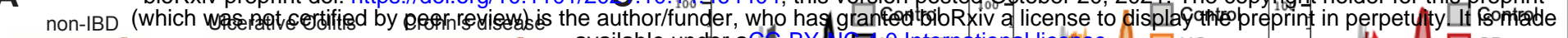
vailable under aCG-BY $N \varangle 4$ Nohntonational license. $\square$ UC

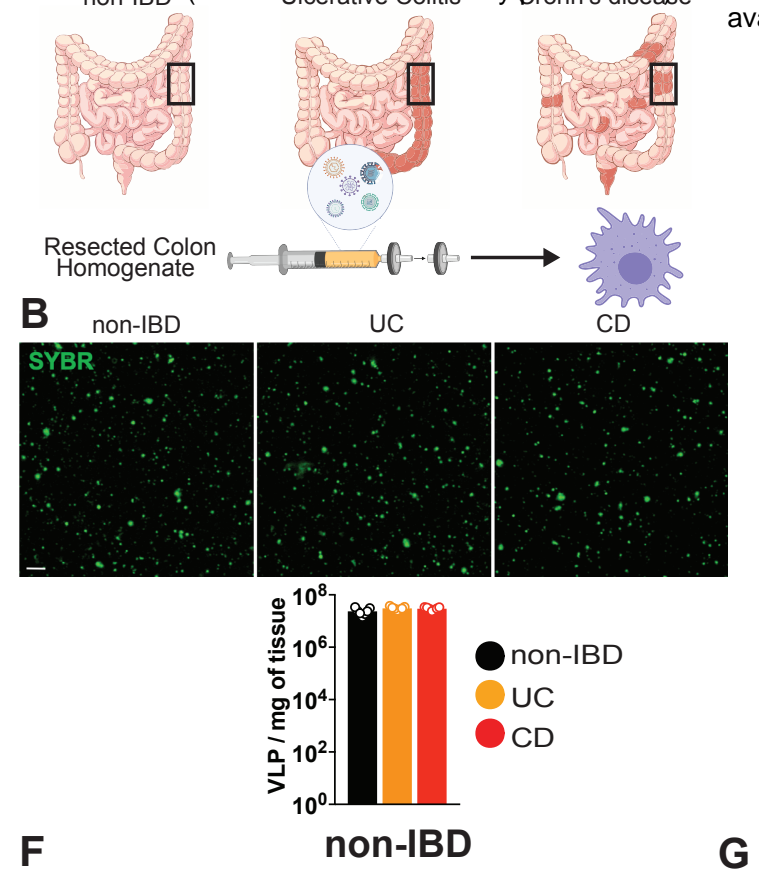

$\mathbf{F}$

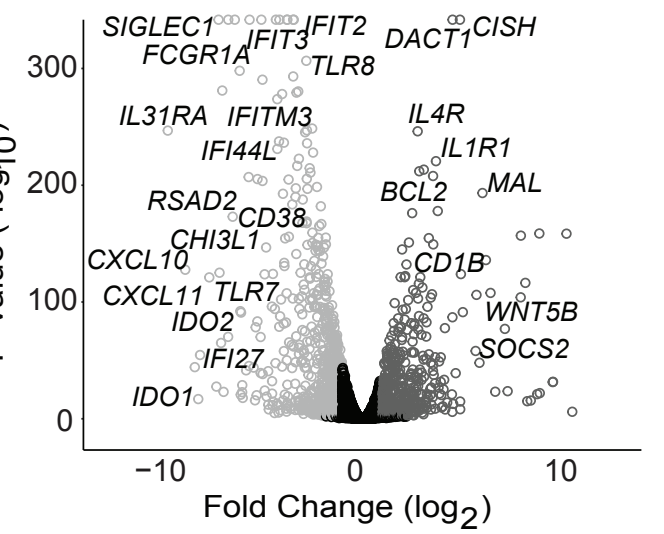

I

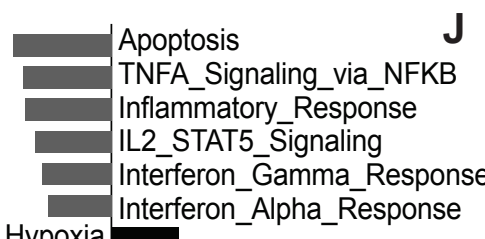

Hypoxia

Estrogen Response Late Estrogen_Response_Early

$$
-2002
$$

$=$

$\frac{\star \star N E S}{* \star}$

Colon lleostomy
WNT BetaC̄atenin Signaling
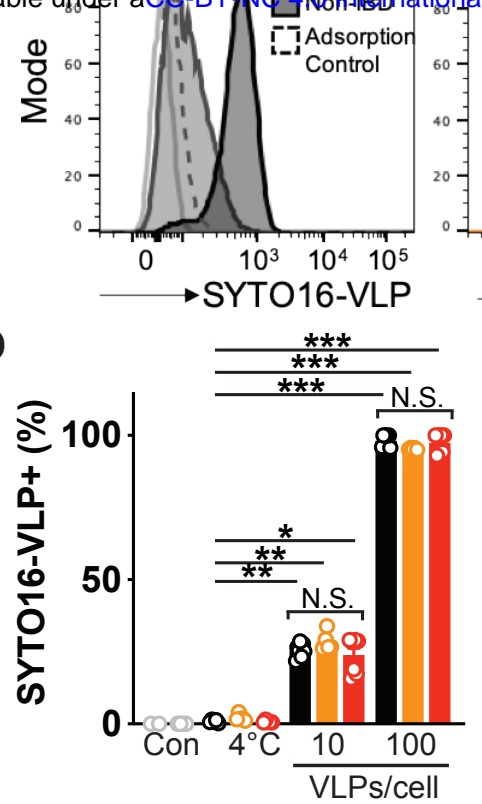

UC

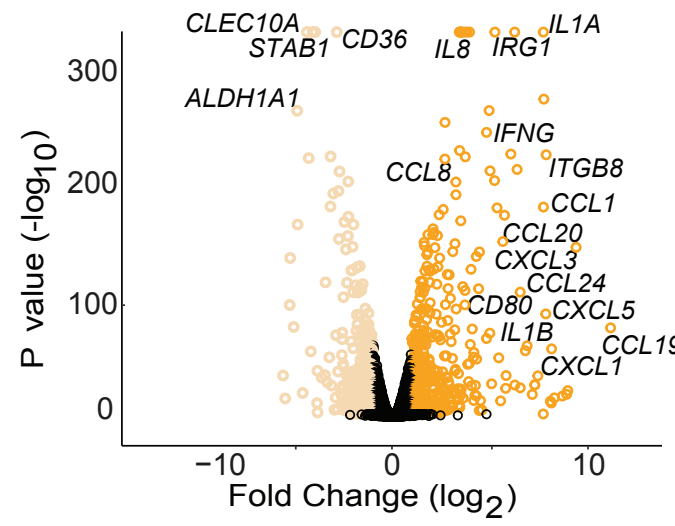

TNFA_Signaling_Via_NFKB

Apoptosis

IL2_STAT5_Signaling

TGF_Beta_Signaling

Hypoxia

Inflammatory_Response

IL6_JAK_STAT3_Signaling
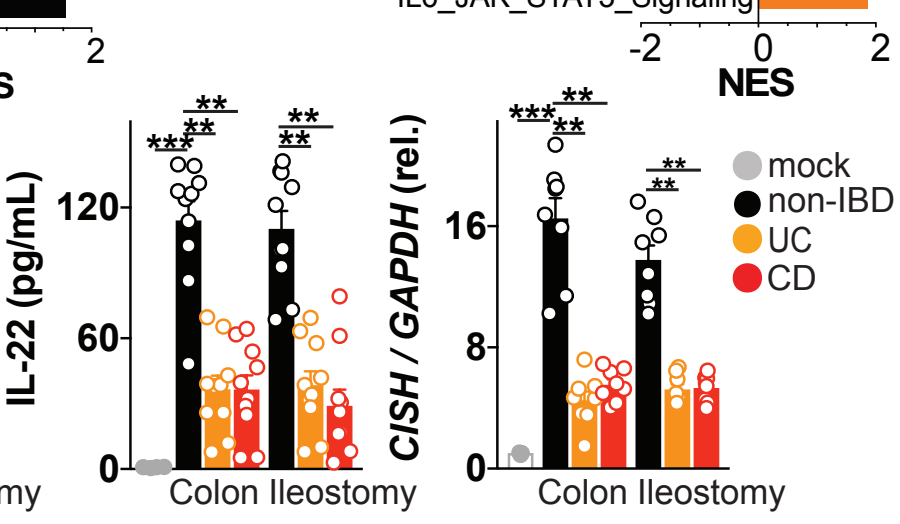

01

Colon Ileostomy$$
\begin{gathered}
\overline{\bar{\varepsilon}} \\
\bar{c} \\
\bar{c} \\
= \\
=
\end{gathered}
$$

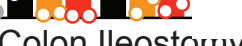

Colon Ileostorny

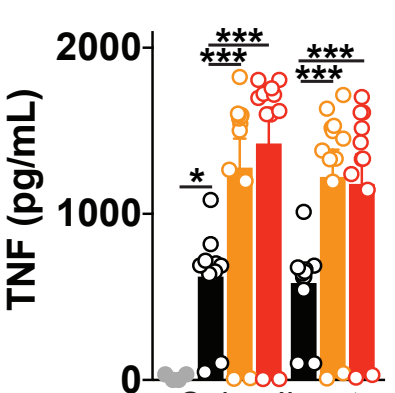

Colon lleostomy

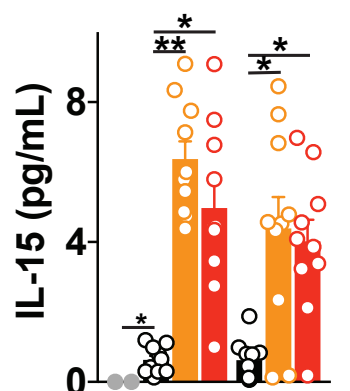

Colon Ileostomy
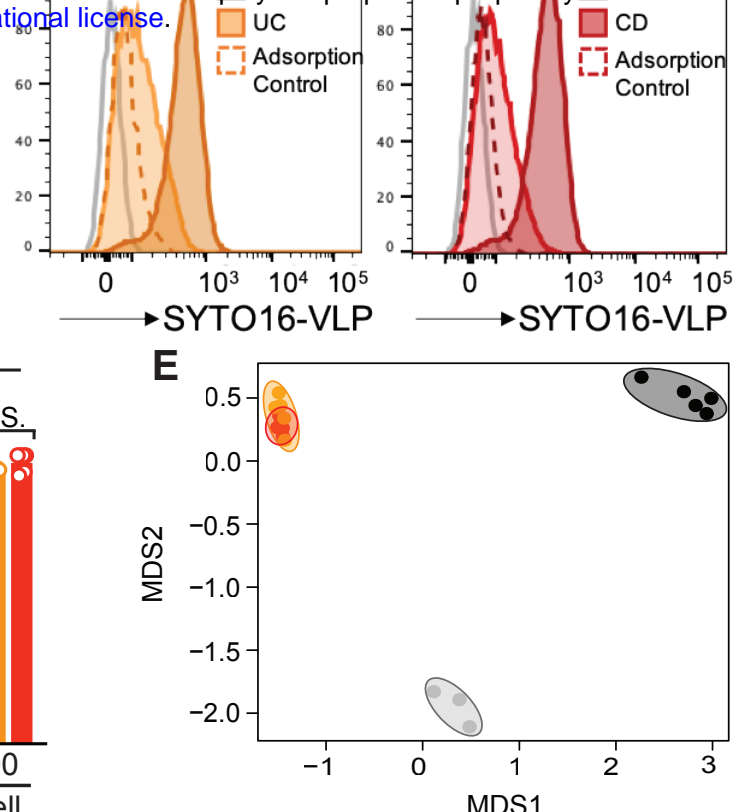

H

CD

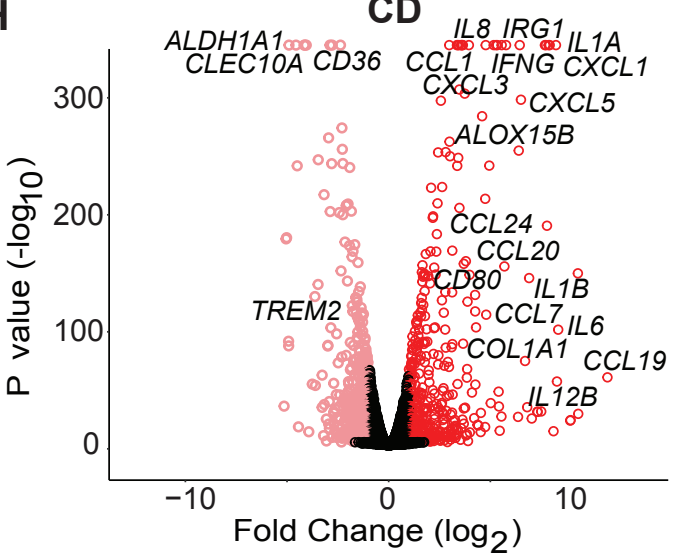

K

Peroxisome Heme_Metabolism Myogenesis

Bile Acid Metabolism Estrogen Response- Late TNFA Signāling Via NFKB IL2_STAT5 Signaling Inflammātory_Response

IL6_JAK_STAT3 Signaling

Apoptosis

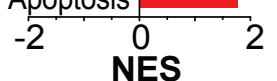

NES

non-IBD VLP

IBD VLP

Onon-IBD+IBD VLP

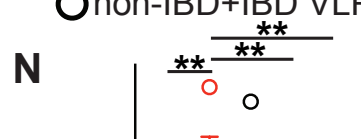

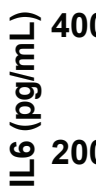
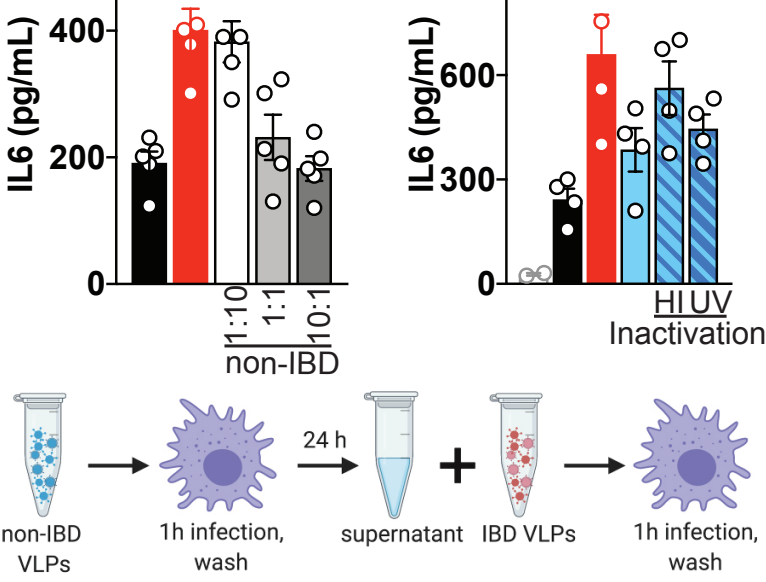
bioRxiv preprint doi: https://doi.org/10.1101/2021.10.14.464404 $\cdot$ this version posted October 20,2021 . The copyright holder for this preprint (which was not certified by peer review) is the author/funder, who has granted bioRxiv a license to display the preprint in perpetuity. It is made available under aCC-BY-NC 4.0 International license.

A

Eukaryotic viruses

Bacteriophages

non-IBD

UC

CD
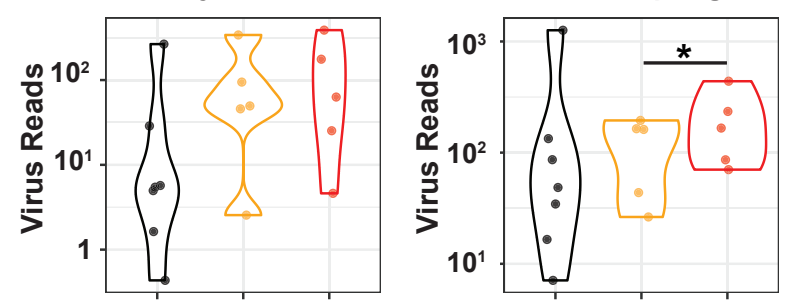

B

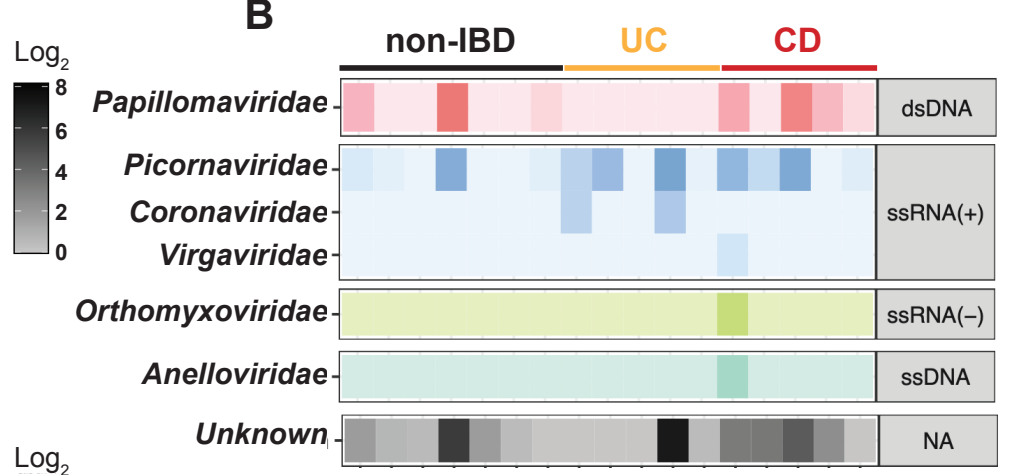

m

10

Siphoviridae-

Myoviridae-

Podoviridae-

Herelleviridae.

Ackermannviridae-

Caudovirales unknown

Lipothrixviridae

Microviridae -

Unknown-

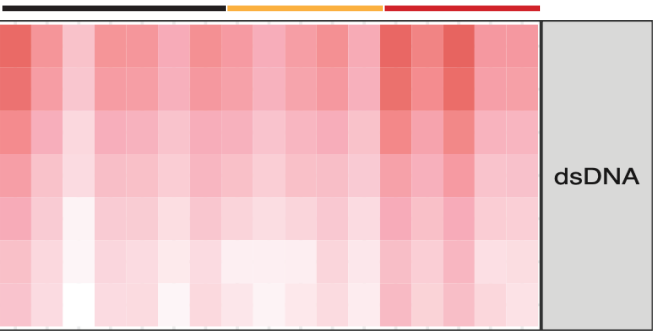

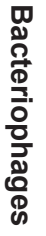

Enterovirus B

C (ssRNA+)
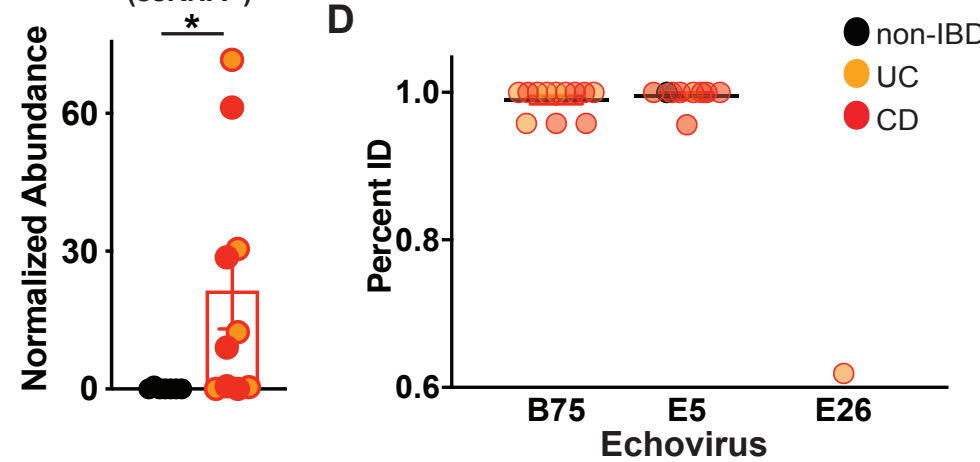

E

F

Siphoviridae, Myoviridae, Vequintavirus

G Gustavvirus (dsDNA) (ssDNA)
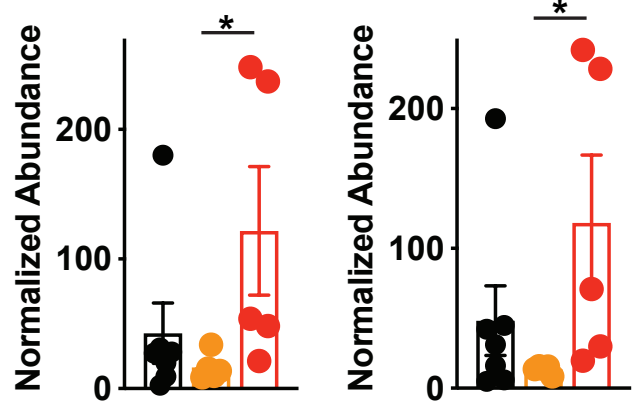

Bruynoghevirus (dsDNA)

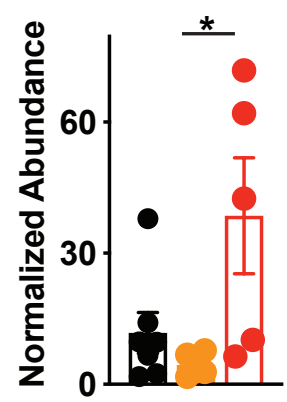


A

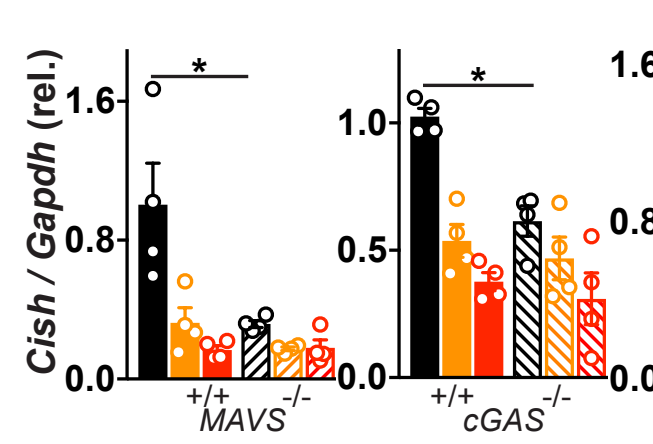

B
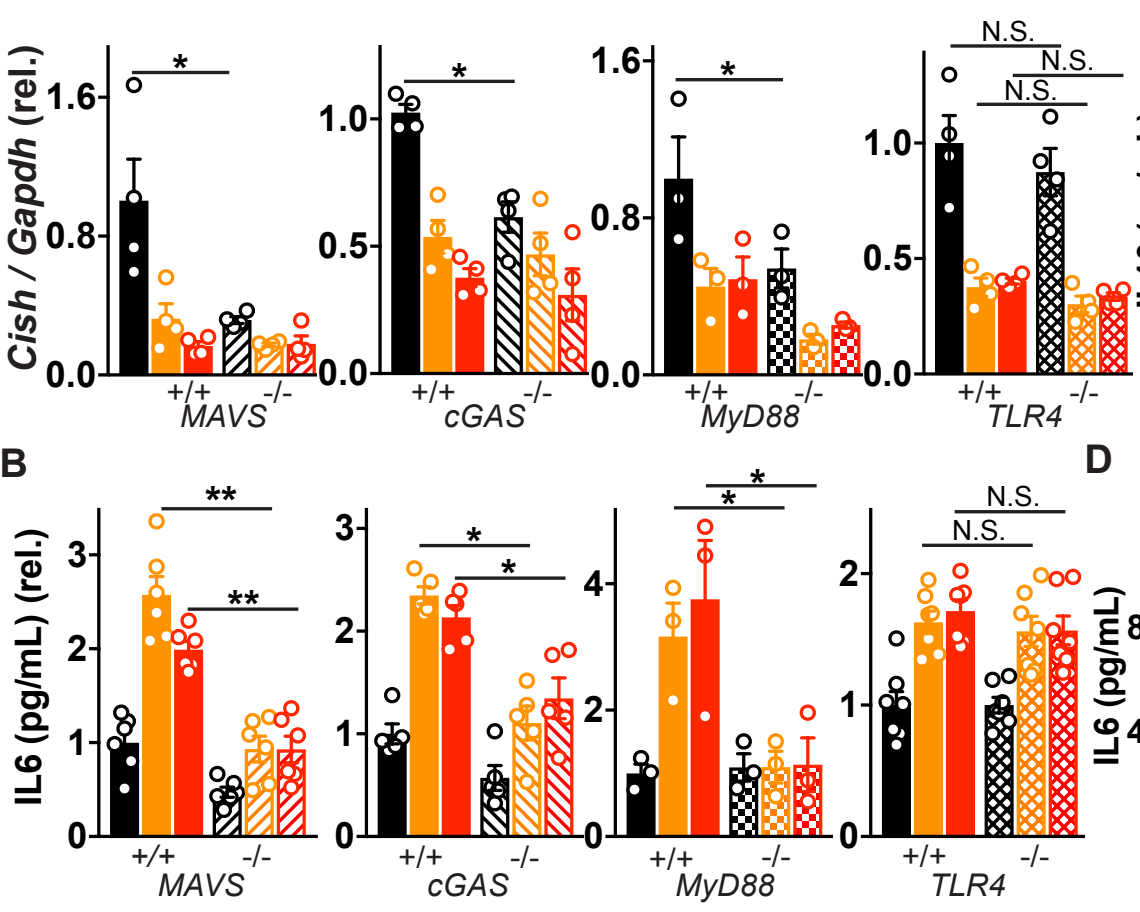

C

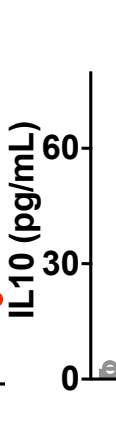

D

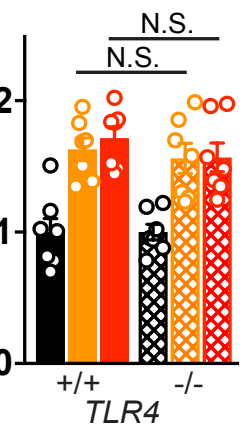

$\mathbf{E}$

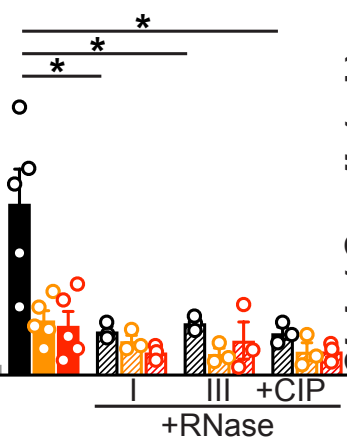

$\mathbf{F}$
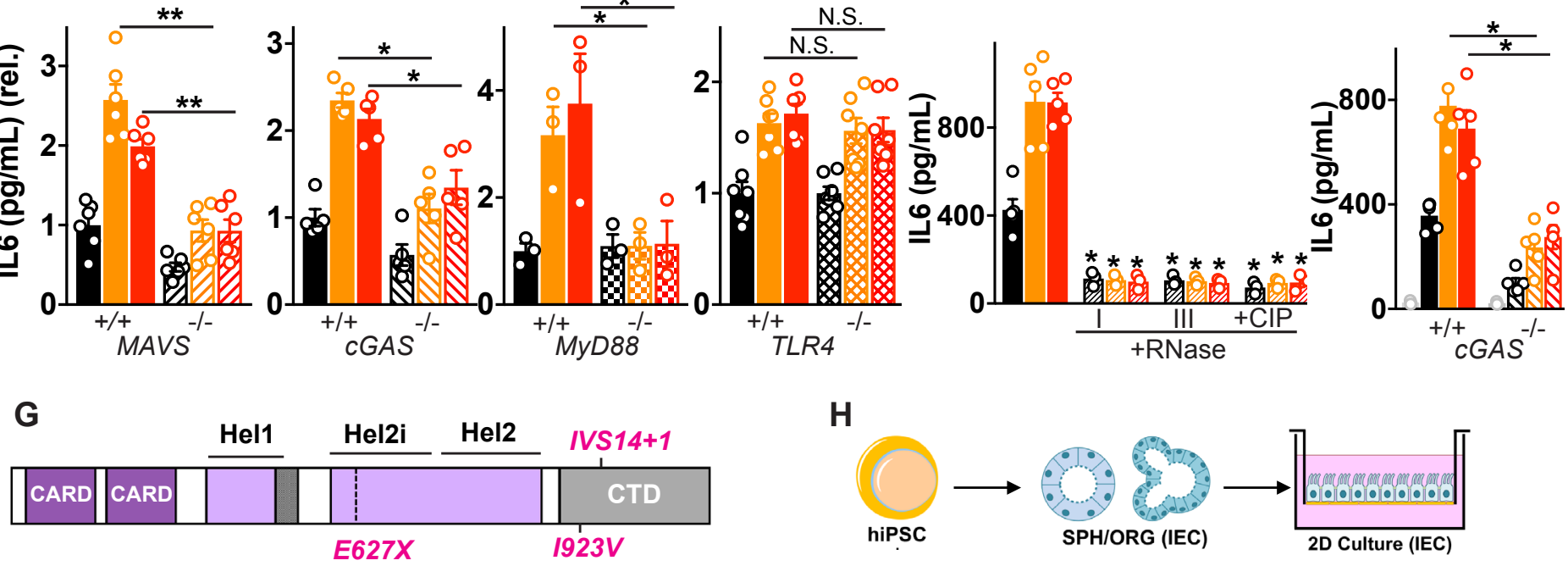

H
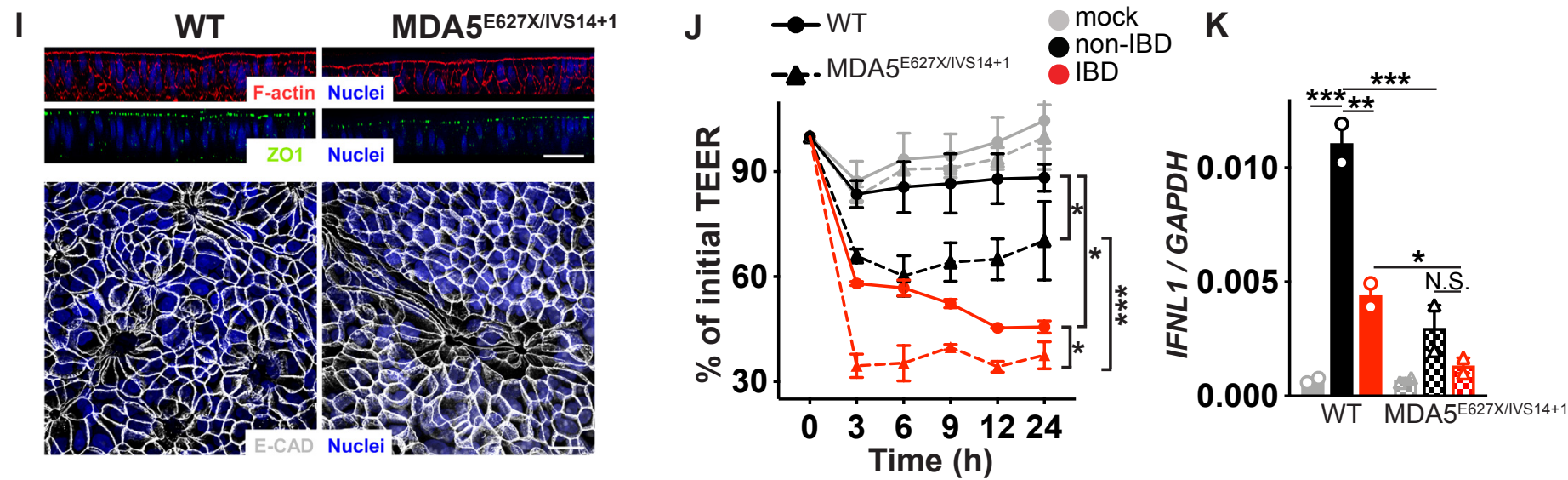


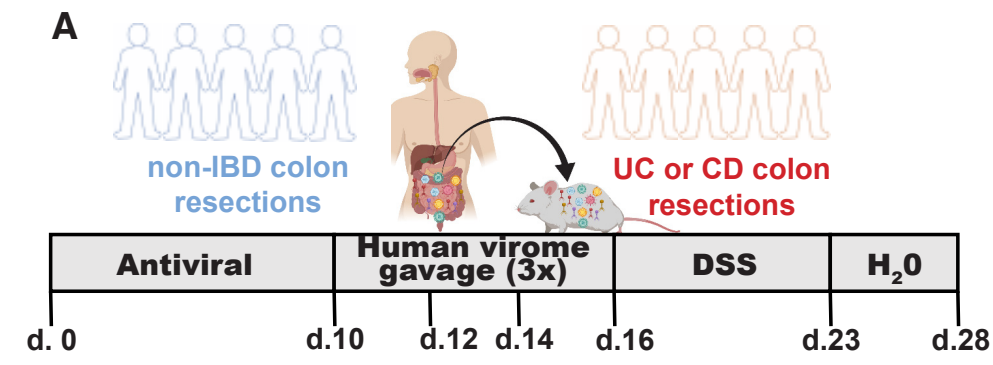

B Gated on live CD45+/MHCII+/CD11b+/CD11C+
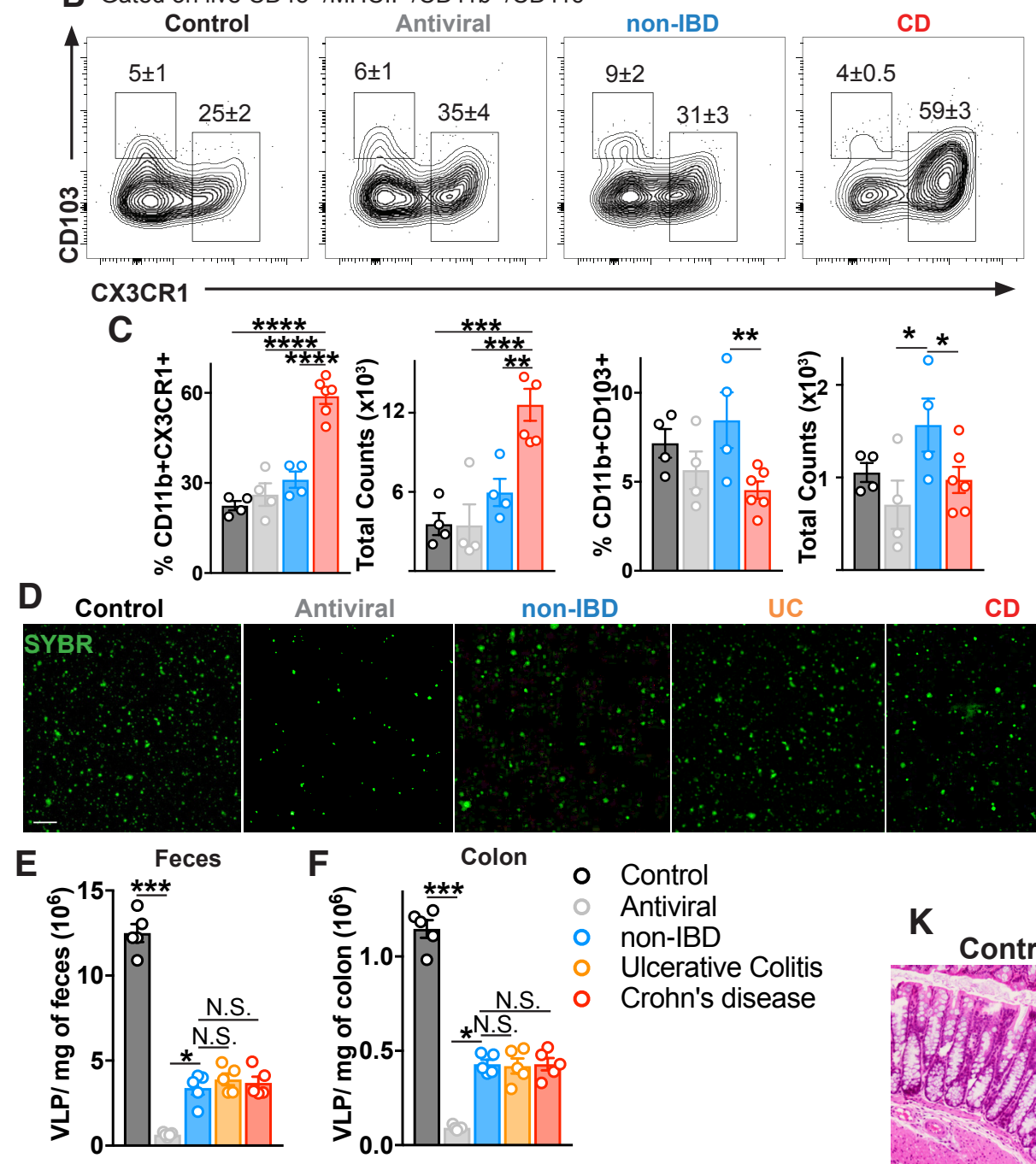

Antiviral

non-IBD
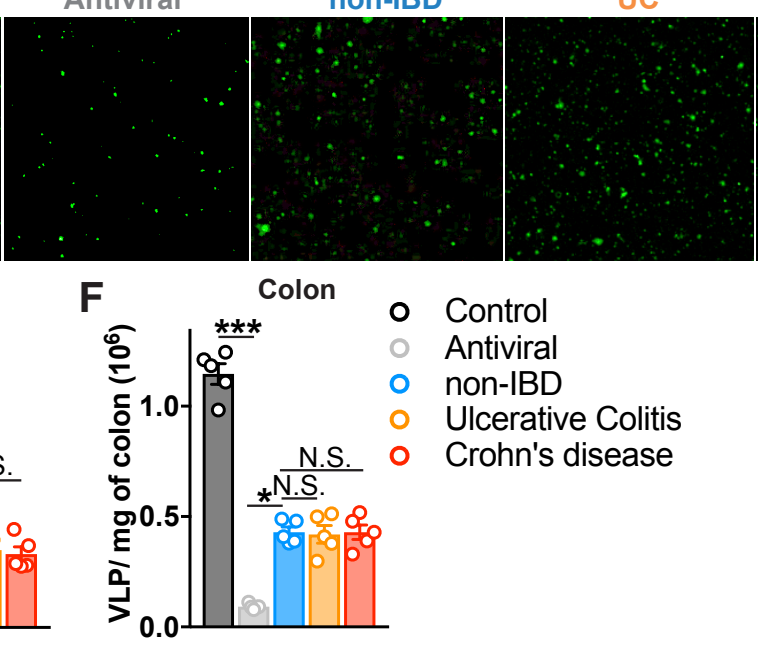

CD
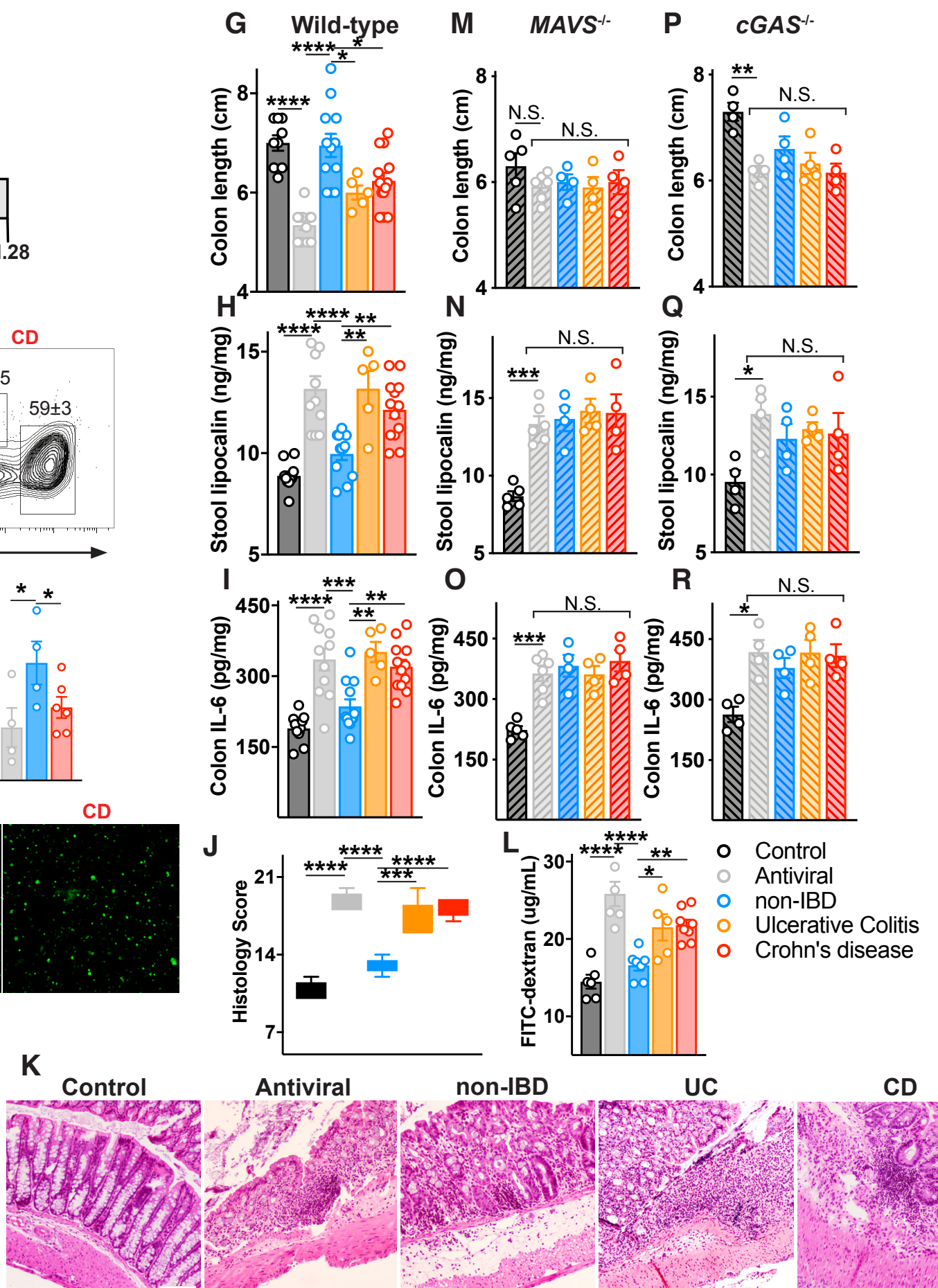

UC

CD 


\section{Supplementary Materials for}

\section{Human enteric viruses shape disease phenotype through divergent innate immunomodulation.}

Authors: Fatemeh Adiliaghdam ${ }^{1}$, Hajera Amatullah ${ }^{1}$, Sreehaas Digumarthi ${ }^{1}$, Tahnee L. Saunders ${ }^{1}$, Raza-Ur Rahman ${ }^{1}$, Lai Ping Wong ${ }^{2,3}$, Ruslan Sadreyev ${ }^{2,4}$, Lindsay Droit ${ }^{5}$, Jean Paquette $^{6}$, Philippe Goyette ${ }^{6}$, John Rioux ${ }^{6,7}$, Richard Hodin ${ }^{8}$, Kathie Mihindukulasuriya ${ }^{5}$, Scott A. Handley ${ }^{5}$, Kate L. Jeffrey ${ }^{1,9^{*}}$

*Correspondence to: KJeffrey@mgh.harvard.edu

\section{This PDF file includes:}

Materials and Methods

Figures S1 to S16

Tables S1 to S5 


\section{Materials and Methods:}

Patient intestinal resections and ileostomy fluid

All human samples were collected under Institutional Review Board (IRB)-approved protocols by Massachusetts General Hospital (MGH) and Research Blood Components, including informed consent obtained in accordance with relevant ethical regulations. Fresh colon resections were collected from patients that were enrolled in the "Prospective Registry in IBD Study at Massachusetts General Hospital (PRISM, IRB\# FWA00003136, Table S1). Study research coordinators obtained consent, and medical history was obtained and confirmed by review of the electronic medical record. Inflammation in UC or CD colon resection samples was confirmed grossly and microscopically by a clinical pathologist. Non-IBD controls included individuals undergoing colon resection surgeries for colorectal cancer, familial adenomatous polyposis (FAP) or Diverticulitis. Lack of inflammation in non-IBD control resection samples was confirmed by a clinical pathologist. Ileostomy fluid was collected from patients enrolled in the "Quantifying the Pro-Inflammatory Nature of Human Fluid Collection Study" that was approved by the Committee for the Protection of Human Subjects at MGH (IRB\#2011P002755, Table S2).

Virus Like Particle (VLP) Isolation

$100 \mathrm{mg}$ of colon tissue or centrifuged ileostomy fluid was resuspended in $500 \mu \mathrm{L}$ of saline magnesium buffer (SM) buffer $\left(8 \mathrm{mM} \mathrm{MgSO}_{4}, 100 \mathrm{mM} \mathrm{NaCl}, 50 \mathrm{mM}\right.$ Tris- $\mathrm{HCl} \mathrm{pH} 7.4$, and $0.002 \%(\mathrm{w} / \mathrm{v})$ gelatin, passed through a $0.02 \mu \mathrm{m}$ Whatman filter), homogenized using sterile metal beads, and centrifuged. The supernatant was then passed through a $0.45 \mu \mathrm{m}$ pore-sized membrane, followed by $0.22 \mu \mathrm{m}$ pore-sized membrane (Millipore). The filtrates were treated with lysozyme $\left(10 \mu \mathrm{g} / \mathrm{mL}\right.$; Sigma) for $30 \mathrm{~min}$ at $37^{\circ} \mathrm{C}$, followed by incubation with 0.2 volumes of chloroform for $10 \mathrm{~min}$ to degrade any remaining bacterial and host cell membranes and then centrifuged at $2,500 \times \mathrm{g}$ for $5 \mathrm{~min}$ at room temperature. The aqueous phase was collected and incubated with DNase I (3U/200 $\mu \mathrm{L}$, Sigma) for $1 \mathrm{~h}$ at $37^{\circ} \mathrm{C}$ to remove any non-virus protected DNA. Enzyme activity was inactivated by incubation at $65^{\circ} \mathrm{C}$ for $15 \mathrm{~min}$ as described (1). To remove any potential residual endotoxin, samples were treated with Polymyxin B $(10 \mu \mathrm{g} / \mathrm{mL}$, Sigma) for 30 minutes. Undetectable endotoxin was confirmed using Limulus Amebocyte Lysate (LAL) assay kit (GenScript) following the manufacturer's instructions.

Quantification of VLPs

Virus-like particles (VLPs) were diluted 10-fold serially, stained for 30 minutes with 10× SYBR Gold (Thermo Fisher Scientific) for nucleic acid or Dialkylcarbocyanine (DiI, Thermo Fisher Scientific) for lipid bilayers of enveloped viruses, and imaged using a Zeiss LSM510 laser scanning confocal microscope. Images were captured using Zeiss software (ZEN). Particles $<0.5 \mu \mathrm{m}$ in diameter were regarded as VLPs. SM buffer was used as a negative control. 10 VLP images were captured per sample and VLPs were counted using an image analyzer (InnerView ${ }^{\mathrm{TM}}$ ).

\section{VLP sequencing}

Total RNA and DNA was extracted from VLPs on a MagNA Pure 24 instrument (Roche), according to the manufacturer's instructions. In order to evaluate samples for both RNA and DNA viruses, the total nucleic acids were randomly amplified as described previously $(2,3)$ using barcoded primers consisting of a base-balanced 16-nucleotide-specific sequence upstream of a random 15-mer and used for NEBNext library construction (New England BioLabs). The 
libraries were multiplexed on an Illumina NextSeq (Washington University Center for Genome Sciences) using the paired-end $2 \times 150$ protocol.

$\underline{\text { Identification and analysis of viral-like sequences }}$

Unprocessed paired-end reads were processed through a multistage quality-control procedure to remove primers and adapters, human and other contaminant and low-quality sequence data. Exact duplicate sequences were removed reserving a single copy and all remaining sequence dereplicated allowing for 4 substitutions. These high-quality / low-redundancy sequences were systematically queried against protein or genomic reference databases using MMseqs2 (4) translated and untranslated search strategies. Sequences assigned a eukaryotic viral or phage taxonomic lineage were first identified using primary searches against virus sequence databases and subsequently confirmed in secondary searches using reference databases with additional non-viral taxonomic lineages. Specific eukaryotic viral families were selected for subsequent analysis by gating on the alignment length and percent identity for each family that separated the high-quality alignments from potentially spurious alignments (Table S3). Only high-quality reads were used for subsequent analysis. Specific phage reads were selected for subsequent analysis by gating on an alignment length of $75 \mathrm{nt}$, to account for the current state of phage taxonomy. In order to determine which phage and eukaryotic viral reads are differentially abundant in the three cohorts, we analyzed the reads at the family, genus and species levels. The non-parametric Kruskal-Wallis test was used to assess the difference in median abundance of eukaryotic viral and phage taxa in the Non-IBD, UC and CD cohorts. The Dunn's post-hoc test was used to correct for multiple comparisons and only those taxa with an adjusted $p$-value $<0.05$ were considered significant. The mean abundance of viral or phage reads, scaled by library size, was determined for each sample in the colon and ileostomy samples. The non-parametric Welch's paired t-test was used to determine which cohorts had significantly different numbers of viral or phage reads.

\section{Human peripheral blood derived macrophages}

Peripheral Blood Mononuclear Cells (PBMCs) were isolated from 20-30 mL blood buffy coats from healthy human volunteers (MGH blood components lab). Briefly, mononuclear cells were isolated by density gradient centrifugation of PBS-diluted buffy coat/blood (1:2) over Ficoll-Paque Plus (GE Healthcare). The PBMC layer was carefully removed and washed 3 times with PBS, mononuclear cells were collected as PBMC. In order to obtain macrophages, PBMCs were resuspended in X-VIVO medium (Lonza) containing 1\% penicillin/streptomycin (Gibco) and incubated at $37^{\circ} \mathrm{C}, 5 \% \mathrm{CO}_{2}$ for $1 \mathrm{~h}$ to adhere to the tissue culture dish. After $1 \mathrm{~h}$, adherent cells were washed 3 times with PBS and differentiated in complete X-VIVO medium containing $100 \mathrm{ng} / \mathrm{mL}$ human M-CSF (Peprotech) for 7 days at $37^{\circ} \mathrm{C}, 5 \% \mathrm{CO}_{2}$. On day 4, cultures were supplemented with one volume of complete X-VIVO medium containing $100 \mathrm{ng} / \mathrm{mL}$ human M-CSF.

\section{Delivery of VLPs to macrophages}

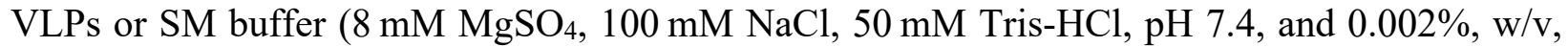
gelatin) were pre-treated for 30 minutes with Polymyxin B (Sigma) to ensure endotoxin-free conditions. Media was removed from $1 \times 10^{6}$ adherent macrophages and VLPs (10 VLPs per cell) or SM buffer ("mock") was added drop-wise onto the cells at a fixed volume sufficient to cover the well surface. VLPs were allowed to adsorb to adherent cells for 1 hour at $37^{\circ} \mathrm{C}$ with occasional rocking. After 1 hour, VLPs were aspirated, cells were gently washed with PBS, and media was replaced (this was considered time $=0$ ). For heat inactivation experiments, VLPs were first 
inactivated for 1 hour at $60^{\circ} \mathrm{C}$. For UV crosslinking of VLPs, VLPs were placed in a UV Stratalinker 2400 from Stratagene on ice to UVB-irradiate three times with $200 \mathrm{~mJ} / \mathrm{cm} 2$ for 15 minutes. For in vitro ratio experiments, non-IBD colon resection-derived VLPs were mixed with $\mathrm{CD}$ colon resection-derived VLPs at increasing ratios to the final concentration of 10 VLPs per cell and VLPs were delivered to macrophages as described above. For supernatant in vitro experiments, the supernatant from macrophages that were exposed to the non-IBD VLP 24 hours prior was mixed with IBD VLPs and added drop-wise onto the cells. VLPs and supernatant mix were allowed to adsorb to adherent cells for 1 hour at $37^{\circ} \mathrm{C}$ with occasional rocking. Heat inactivated $\left(95^{\circ} \mathrm{C}\right.$ for $\left.10 \mathrm{~min}\right)$ or $\mathrm{UV}$ crosslinked (three times $200 \mathrm{~mJ} / \mathrm{cm} 2$ for 15 minutes) supernatant were used as controls.

\section{Fluorescent labeling of VLPs and uptake assays}

Purified, quantified colon resection derived VLP preparations were labeled with $0.01 \mu \mathrm{M} \mathrm{SYTO}^{\mathrm{TM}}$ 16 Green Fluorescent Nucleic Acid Stain (Thermo Fisher Scientific, \#S7578) according to manufacturer's instructions. Labeled VLPs were then separated from unincorporated dye using PD10 Sephadex G-25 desalting columns (GE Healthcare, \#17085101) according to manufacturer's instructions. Uptake assays of SYTO16-labeled VLPs were performed with human peripheral blood monocyte derived macrophages. Cells were seeded at a density of $10^{5}$ cells/well in a 24 -well plate. Cells were delivered $10^{6}$ or $10^{7}$ non-IBD, UC or CD VLPs or SM buffer control by adsorption in $100 \mu \mathrm{L}$ for $1 \mathrm{~h}$, washed and left for 3 hours at $37^{\circ} \mathrm{C}$ for uptake analysis or $4^{\circ} \mathrm{C}$ for adsorption analysis. Cells were removed from the culture plate surface with cold PBS, washed in flow cytometry buffer twice, stained for live-dead discrimination and acquired on an LSR II flow cytometer (BD Biosciences). For the inhibition assays, cells were treated with $3.0 \mathrm{mg} / \mathrm{mL}$ of Brefeldin A (Invitrogen, $\# 00-4506$ ) for $30 \mathrm{~min}$ at $37^{\circ} \mathrm{C}$ before addition of labeled VLPs and during the adsorption. Data was analyzed using FlowJo v10 software (TreeStar).

$\underline{\text { RNA sequencing and analysis }}$

Poly(A)-mRNA was enriched using the NEBNext Poly(A) mRNA magnetic isolation module (NEB), according to manufacturer's instructions. Libraries were then generated using the NEBNext Ultra II RNA Library Prep Kit for Illumina (NEB). To allow for multiplexing of samples, libraries were dual indexed using indices from NEBnext Multiplex Oligos for Illumina (NEB). Paired-end sequencing was performed using a NovaSeq 6000. Samples were run in replicate across three separate lanes. Transcriptome mapping was performed with STAR version 2.5.4a(5) using the NCBI37 assembly gene annotations. Read counts for individual genes were produced using the unstranded count feature in HTSeq 0.9.1(6). Differential expression analysis was performed using the EdgeR package(7) after normalizing read counts and including only those genes with count per million reads $(\mathrm{cpm})$ greater than 1 for one or more samples $(8)$. Differentially expressed genes (DEG) were defined based on the criteria of minimum 2-fold change in expression value and false discovery rate (FDR) less than 0.05. Gene Set Enrichment Analysis (GSEA) (9) was adopted for functional annotation analysis.

VLP RNA or DNA isolation

For VLP RNA or DNA transfection experiments, VLP preparations were mixed with Trizol-LS (Invitrogen) and RNA and DNA fractions isolated. DNA was further purified by ethanol precipitation. The RNA fraction was subjected to RNeasy Mini Kit (Qiagen) with on-column DNase I digest for clean-up and removal of residual DNA. For enzyme treatments of nucleic acids, 
$2.5 \mu \mathrm{g}$ of RNA was treated with RNase I ( $5 \mu \mathrm{g} / \mathrm{mL}$, Ambion) or RNase III ( $5 \mu \mathrm{g} / \mathrm{mL}$, Ambion) or with Calf Intestinal Phosphatase (CIP, 250U, Roche) at $37{ }^{\circ} \mathrm{C}$ for $1 \mathrm{~h}$. Enzyme-treated RNA was purified with RNeasy Mini Kit (Qiagen) before transfection. Human macrophages were transfected with $2.5 \mu \mathrm{g}$ of RNA or DNA using Lipofectamine 2000 (Thermo Fisher Scientific) according to the manufacturer's protocol.

\section{ELISA}

Cell culture supernatants were removed from human macrophages and centrifuged to remove debris and non-adherent cells. Secreted cytokines were measured using a human Cytokine 25-Plex Magnetic Panel for Luminex® Platform (ThermoFisher, EPX250-12166-901) and read on a Luminex FLEXMAP $3{ }^{\circledR}$ instrument. Human and mouse TNF, IL-6, IFN- $\beta$, IL-8, IL-15, IL-10 and IL-22 ELISA kits (R\&D) were also used. Supernatants and kit-supplied cytokine standards were processed in triplicate according to the manufacturer's instruction.

\section{Quantitative PCR}

RNA was extracted using the RNeasy Mini Kit (Qiagen) with on-column DNase digest (Qiagen) according to manufacturer's instruction. 100ng-1 $\mu \mathrm{g}$ RNA was used to synthesize cDNA by reverse transcription using the iScript cDNA Synthesis Kit (Bio-Rad). Quantitative PCR reactions were run with cDNA template in the presence of $0.625 \mu \mathrm{M}$ forward and reverse primer and 1x solution of iTaq Universal SYBR Green Supermix (Bio-Rad). Quantification of transcript was normalized to the indicated housekeeping gene. A complete list of primer sequences is provided in Supplementary Table 4.

\section{Human Caco2 cells monolayer trans-well model}

Human Caco 2 epithelial cells were purchased from the American Type Culture Collection (ATCC) and maintained in DMEM with 10\% heat-inactivated fetal bovine serum (FBS) and 1\% PenicillinStreptomycin (P/S) solution. Toll-like Receptor 4 (TLR4)-null Caco2 cells were kindly provided by Dr. Richard Hodin's lab (Massachusetts General Hospital). For creating the monolayer, wildtype or TLR4-null Caco2 cells were seeded onto collagen-coated polyethylene terephthalate (PET) filter supports ( $1 \mu \mathrm{m}$ pore size, Corning) at a concentration of $3 \times 10^{5}$ cells/well and were incubated $\left(37^{\circ} \mathrm{C}, 5 \% \mathrm{CO}_{2}\right)$ for $2-3$ weeks. Both the apical and the basolateral side of the trans-wells were fed with DMEM supplemented with $10 \% \mathrm{FBS}$ and $1 \% \mathrm{P} / \mathrm{S}$ solution. After 2-3 weeks the integrity of the monolayer was measured by transepithelial electrical resistance measurements (TEER) using the Millicell-ERS electrical resistance measuring system (Millipore). The electrodes were immersed in a way that the shorter electrode was in the inner well and the longer electrode was in the outer well. $200-220 \Omega . \mathrm{cm}^{2}$ resistance was indicated as a confluent monolayer. When TEER peak was reached, and maintained for 3 days, the monolayer was used for subsequent experiments. SM buffer and LPS (100 ng/mL) were used as negative and positive controls, respectively. Apical compartment surface was incubated with VLPs whereby VLPs were allowed to adsorb to adherent cells for 1 hour at $37^{\circ} \mathrm{C}(10$ VLPs per cell). After 1 hour, VLPs were aspirated, cells were gently washed with PBS, and fresh media was replaced (this was considered time $=0$ ). The cells were then returned to the cell culture incubator $\left(37^{\circ} \mathrm{C}, 5 \% \mathrm{CO}_{2}\right)$ and TEER measured every 6 hours up to 48 hours. All experiments were performed in triplicate.

\section{HEK293T IFN $\beta$ luciferase reporter assay}


HEK293T cells were maintained in 48-well plates in Dulbecco's modified Eagle medium (Cellgro) supplemented with $10 \%$ FBS and 1\% penicillin/streptomycin. Cells were transfected with $10 \mathrm{ng}$ of indicated FLAG-MDA5 plasmids (kindly provided by the T. Fujita, Kyoto University), IFNBpromoter driven firefly luciferase reporter plasmid (100 ng) and a constitutively expressed Renilla luciferase reporter plasmid (pRL-CMV, $10 \mathrm{ng}$ ) by using Lipofectamine2000 (Life Technologies), according to the manufacturer's protocol. Empty vector was used to maintain a constant total amount of DNA and lipofectamine. Media was changed 6 hours after the first transfection and cells were additionally transfected with $1 \mu \mathrm{g} / \mathrm{mL}$ of RNA isolated from either non-IBD virus like particles (VLPs), polyinosinic:polycytidylic acid (poly I:C, Sigma), or RNA isolated from the cytoplasm of HEK-293T cells (self RNA). Cells were lysed $24 \mathrm{~h}$ post-stimulation and IFN $\beta$ promoter activity was measured using the Dual Luciferase Reporter assay (Promega). Firefly luciferase activity was normalized against Renilla luciferase activity.

Differentiation of human iPSC into intestinal epithelial cells

Two lymphoblastoid cell lines (LCL) were selected from the NIDDK IBDGC Repository based on their IFIH1 genotypes: (1) an LCL line carrying wild-type IFIH1 alleles from a 39 year-old Female Caucasian and (2) an LCL line that is a compound heterozygote for rs35732034 (IVS14+1) and rs35744605 (E627X) from a Caucasian 47 year-old male Ulcerative Colitis patient. Specifically, the risk allele at rs35732034 (IVS14+1) is a splice donor site substitution at position +1 in intron 14 that leads to exon 14 skipping and the truncation of the C-terminal domain, and the risk allele at rs35744605 (E627X) is a nonsense variant leading to the loss of the C-terminal half of the protein $(10,11)$. The two LCL lines were then reprogrammed to human induced pluripotent stem cells (iPSCs) as described (12) via nucleofection with four episomal reprogramming plasmids (pCE-hUL, pCE-hSK, pCE-hOCT3/4, and pCE-mp53DD) and selection of TRA-1-81 positive reprogrammed hiPSC colonies. LCL-derived hiPSC were then used to generate polarized epithelial monolayers using a stepwise differentiation protocol involving a conversion to definitive endoderm (DE) using the STEMdiff Definitive Endoderm kit (StemCell Technologies). This was followed by the formation of hindgut (HG) structures with a treatment of $3 \mu \mathrm{M} \mathrm{CHIR} 99021$ and $250 \mathrm{ng} / \mathrm{ml} \mathrm{FGF} 4$ for 3-4 days, as previously described (13). HG structures were harvested and then centrifuged at $100 \mathrm{~g}$ for 5 min, embedded in Matrigel (Corning) and cultured for 15 days in Intestinal Growth Medium with feeding every 2-3 days as described (14) but using human recombinant inducers rhWnt3a (Cedarlane), rhEGF (Cedarlane), rhNoggin (Cedarlane), rhRSpondin-1 (Millipore-Sigma) and rhHGF (Cedarlane). Following this, the spheroids were cultivated in human organoid culture medium (HOCM) and passaged every 6-8 days for maturation into 3D Intestinal epithelial cell (IEC) cultures of spheroids (SPH) and organoids $(\mathrm{ORG})$ as described $(13,14)$. Intestinal epithelial cell structure and function was then assessed (see Supplementary Fig 14).

Delivery of Virus like Particles (VLPs) to hiPSC-derived intestinal epithelial cell monolayers Spheroids were recovered from the Matrigel by treatment with Cell Recovery Solution (Corning), dissociated in TrypLE Express for $20 \mathrm{~min}$ at $37 \mathrm{C}$ and further dissociated by pipetting. A typical confluent Matrigel drop of spheroid was used for five Transwell inserts (about $2 \times 10^{5}$ cells). The cell suspensions were centrifuged at $450 \mathrm{~g}$ for $5 \mathrm{~min}$ and resuspended in HOCM $(100 \mu \mathrm{L}$ per Transwell). Cells were seeded on the apical side (upper chamber) of the collagen-coated Transwell unit (PTFE COL $0.4 \mu \mathrm{m} 0.33 \mathrm{~cm}^{2}$, Corning) and $600 \mu \mathrm{L}$ of HOCM was added to the bottom chamber. Transepithelial Electrical Resistance was measured every 2-3 days using Epithelial 
Voltohmmeter (EVOM2, World Precision Instruments, LLC) until a plateau was reached (similar TEER value on three consecutive measurements). Cells on the apical compartment surface were infected with VLPs (MOI 10) as described above. VLPs were allowed to adsorb to adherent cells for 1 hour at $37^{\circ} \mathrm{C}$. After 1 hour, VLPs were aspirated, cells were washed with PBS, and fresh media was replaced. SM buffer and poly I:C $(50 \mu \mathrm{g} / \mathrm{mL})$ were used as negative and positive controls, respectively. The cells were then returned to the cell culture incubator $\left(37^{\circ} \mathrm{C}, 5 \% \mathrm{CO}_{2}\right)$ and the integrity of the monolayer was measured by transepithelial electrical resistance measurements (TEER) every 6 hours up to 24 hours.

\section{MDA5 expression in epithelial cells}

\section{Western Blot}

Mouse intestines were harvested and pre-digested in $1 \mu \mathrm{M}$ ethylenediaminetetraacetic acid (EDTA) and $3 \mu \mathrm{M}$ dithiothreitol (DTT) at $37^{\circ} \mathrm{C}$ for 15 minutes with shaking (400 rpm) to isolate epithelial cells. Remaining tissue was minced and digested with collagenase type II (Gibco) for 45 minutes at $37^{\circ} \mathrm{C}$ with constant agitation followed by Percoll density-gradient centrifugation to isolate single cells from the lamina propria. Cells were incubated with biotinylated anti-CD45 to purify the hematopoietic cell population. Beads were gently washed, and bound cells isolated by MACS mini-column purification using streptavidin magnetic beads. Epithelial cells and lamina propria hematopoietic cells were lysed in $200 \mu \mathrm{L}$ of RIPA buffer (Tris-HCl, pH 7.4, $50 \mathrm{mM}, \mathrm{NaCl} 150 \mathrm{mM}$, NP-40 $1 \%$, sodium deoxycholate $0.5 \%$, SDS $0.1 \%$ ) with a cocktail of protease and phosphatase inhibitors (1\%, Roche). $20 \mu \mathrm{g}$ of protein was loaded per lane and separated on a 4-12\% SDS/PAGE gel and transferred to PVDF. Ponceau stain (Sigma) confirmed protein transfer, and the membrane was blocked for $1 \mathrm{~h}$ at $25^{\circ} \mathrm{C}$ with $5 \%$ skim milk in TBS $/ 0.1 \%$ Tween-20 (TBS-T), and then incubated overnight at $4^{\circ} \mathrm{C}$ with the indicated primary antibodies (diluted 1:1000 v/v in TBS-T with $3 \%$ BSA). Primary antibody was washed off and the membrane was incubated with the indicated secondary antibodies (diluted 1:10000 in TBS-T). The following primary antibodies were used: anti-MDA5 antibody (Sigma), $\beta$-actin (Sigma) was used as a loading control, and Villin (Santa Cruz) was used to confirm purity of epithelial cells.

\section{Immunofluorescence microscopy}

The ileum was fixed with 4\% paraformaldehyde. After antigen retrieval (Sigma, Citrate Buffer, \#C9999), sections were treated with autofluorescence blocking MaxBlock, blocked in 5\% normal goat serum with 1\% BSA in PBS (Blocking buffer) for 1 hour at RT, and stained with the primary antibody anti-MDA5 (Sigma; \#SAB3500356) overnight at $4^{\circ} \mathrm{C}$ at a final concentration of 1:500. Slices were washed and incubated with goat anti-rabbit secondary antibody for 1 hour at RT at a final concentration of 1:500. The slices were washed, counterstained with DAPI (Vector), and mounted for confocal microscopy analysis. All images were collected using a confocal microscope (Nikon A1) (400x).

Mice

Five- to six-week-old female C57BL/6 mice, MAVS-/-, cGAS-/- or TLR4-/- (C3H/HeJ)

were purchased from The Jackson Laboratory. All mice were housed in specific pathogen-free conditions according to the National Institutes of Health (NIH), and all animal experiments were conducted under protocols approved by the MGH Institutional Animal Care and Use Committee (IACUC), and in compliance with appropriate ethical regulations. For all experiments, agematched mice were randomized and allocated to experimental group, with 5-8 mice per group, and 
repeated three independent times. No statistical method was used to determine sample size. For "humanized" virome mice, mice were first depleted of viruses using an antiviral cocktail (Acyclovir $20 \mathrm{mg} / \mathrm{kg}$, Lamivudine $10 \mathrm{mg} / \mathrm{kg}$, Ribavirin $30 \mathrm{mg} / \mathrm{kg}$, Oseltamivir $10 \mathrm{mg} / \mathrm{kg}$ ) by daily gavage for 10 days as described (15). AV-treated mice were then gavaged with $200 \mu \mathrm{L}$ of SM buffer as a control or $4 \times 10^{8}$ VLPs isolated from non-IBD, UC or CD patient fresh colon resections. Mice received the human VLP gavage every other day for three times in total. To ensure that the gastric $\mathrm{pH}$ would not affect the viability of the gavaged VLPs, each mouse was gavaged with 100 $\mu \mathrm{L}$ of $1 \mathrm{M} \mathrm{NaHCO}_{3} 15$ min prior to VLP gavage to keep gastric $\mathrm{pH}$ at 7.4. Quantification of viruses in different treatment groups was performed by confocal imaging of VLPs isolated from feces and colon tissue.

\section{Bone marrow-derived macrophages}

Bone marrow-derived macrophages (BMDMs) were produced from MAVS-deficient, cGASdeficient or TLR4-deficient mice (obtained from The Jackson Laboratory) and their respective littermate controls. Briefly, bone marrow was flushed from tibia and femur and allowed to adhere to a non-treated tissue culture plate for 1 day. Non-adherent cells were then differentiated to macrophages in DMEM containing 10\% FBS, 1\% L-Glutamine, 1\% penicillin/streptomycin, $0.1 \%$ $\beta$-mercaptoethanol, $5 \mathrm{ng} / \mathrm{mL}$ of interleukin 3 (IL-3, Peprotech) and Macrophage Colony Stimulating Factor (M-CSF, Peprotech) for 7 days. Macrophage maturity was assessed by surface expression of CD11b and F4/80 with flow cytometry. MyD88-/- immortalized macrophages were obtained from Kate Fitzgerald (UMass Medical School).

\section{Isolation of lamina propria cells for flow cytometry}

Colons were harvested from mice and caeca were removed. The tissue was placed in RPMI containing 5\% fetal bovine serum (FBS) and fat was removed by careful dissection followed by gentle rolling along a moist paper towel. The tissue was washed gently then inverted and rinsed to remove loose fat and fecal matter. Pieces of the colon were transferred to a solution of RPMI containing $2 \% \mathrm{FBS}, 1 \mu \mathrm{M}$ ethylenediaminetetraacetic acid (EDTA), $3 \mu \mathrm{M}$ dithiothreitol (DTT) and incubated at $37^{\circ} \mathrm{C}$ for 15 minutes with shaking $(400 \mathrm{rpm})$. Next, tissue pieces were rinsed with fresh FBS-containing media to wash off EDTA/DTT and tissue was again rolled gently along a moist paper towel. Tissue was cut into very small pieces and transferred to a digestion medium: each sample was placed in $25 \mathrm{~mL}$ of RPMI media containing $2 \%$ FBS and mixed with $12.5 \mathrm{mg}$ Dispase II (Gibco) and 37.5mg type IV collagenase (Worthington). Digestion was allowed to take place for 45 minutes at $37^{\circ} \mathrm{C}$ with constant agitation, and halfway through this period, tissue was disrupted further by pipetting. After 45 minutes, the digestion solution was pipetted again and smashed through a $100 \mu \mathrm{m}$ mesh. Digestion was quenched by addition of $25 \mathrm{~mL}$ of RPMI containing 5\% FBS. The suspension was spun down for 10 minutes at $450 \mathrm{~g}$ and the resulting pellet was resuspended in fresh media, passed through a $40 \mu \mathrm{m}$ mesh, washed with additional fresh media and spun down again. The final pellet represented the lamina propria fraction of the colon. Cells were blocked, stained, and washed for acquisition on an LSRII flow cytometer (BD). Antibodies used for flow cytometry can be found in Supplementary Table 5. All data was analyzed using FlowJo v10 software (TreeStar).

\section{Dextran Sodium Sulfate induced colitis (DSS)}

Mice were administered 2.5\% Dextran Sodium Sulfate Salt (DSS, MW = 36,000-50,000 Da; MP Biomedicals) in drinking water ad libitum for 7 days (freshly prepared every other day), followed 
by regular drinking water for 5 days. Mice were sacrificed at day 12. Colon length was measured and fecal samples were homogenized in PBS, spun down and supernatants were collected and assayed for Lipocalin-2 by ELISA according to manufacturer's instructions (R\&D). To assess intestinal permeability, mice were given FITC-dextran tracer (Sigma, $4 \mathrm{kDa}, 0.6 \mathrm{mg} / \mathrm{g}$ body weight) intragastrically in $0.2 \mathrm{~mL}$ PBS and FITC levels were measured $4 \mathrm{~h}$ later in hemolysis-free serum using a Fluorescence Spectrophotometer. For cytokine measurements, day 12 colon tissue (approximately $1 \mathrm{~cm}$ ) was washed in PBS and then placed in a 24 well plate containing $750 \mu \mathrm{L}$ of RPMI medium with $1 \%$ penicillin/streptomycin and incubated at $37^{\circ} \mathrm{C}$ with $5 \% \mathrm{CO}_{2}$ for 24 hours. Supernatants were collected and centrifuged for $10 \mathrm{~min}$ at $4^{\circ} \mathrm{C}$ and IL- 6 levels were assessed by ELISA according to manufacturer's instructions (R\&D). For histology, tissue was collected from the distal part of the colon and hematoxylin and eosin staining was performed. Histologic evaluation performed in a blinded fashion and scored based on the criteria outlined previously (16). Three main categories were used to reflect the severity of histopathology: (i) inflammatory cell infiltrates, (ii) epithelial changes and (iii) mucosal architecture.

$16 \mathrm{~S}$ sequencing and analysis

QIAamp DNA Stool Mini Kit was used to extract DNA from feces. The hypervariable region (V4) of the 16S rRNA gene sequences were amplified by using PCR with adaptor primer set containing $12 \mathrm{bp}$ multiplex identifier sequences (Integrated DNA Technologies, Coralville, IA). Each reaction contained template DNA, each primer $(0.2 \mu \mathrm{M})$, and $2 \times$ Platinum $^{\mathrm{TM}}$ Hot Start PCR Master Mix (Invitrogen, Carlsbad, CA). PCR conditions were as follows: $94^{\circ} \mathrm{C}$ for $3 \mathrm{~min}$, followed by 22 cycles at $94^{\circ} \mathrm{C}$ for $45 \mathrm{sec}, 52^{\circ} \mathrm{C}$ for $1 \mathrm{~min}$, and $72^{\circ} \mathrm{C}$ for $1.5 \mathrm{~min}$ and a final extension step at $72^{\circ} \mathrm{C}$ for $10 \mathrm{~min}$. Triplicate reactions were prepared, pooled, and purified using SpeedBead ${ }^{\mathrm{TM}}$ carboxylate magnetic bead solution (GE Healthcare, Marlborough, MA). The amplicons were quantified using KAPA Library Quantitation kit (KAPA, Cape Town, South Africa) and an equimolar amount of each sample was sequenced on the MiSeq System (Illumina, San Diego, CA) with 2x150 paired-end run parameters. Microbiome bioinformatics analysis was performed with QIIME 2 release 2018.11 (17). In brief, cutadapt was used to trim 515F/806R primers in the paired end sequences. Subsequently, the sequences were stripped of noise by using the DADA2 (18) denoiser program. The denoised, trimmed and higher quality amplicon sequence variants (ASVs) were aligned with MAFFT (19) and used to construct a phylogeny with fasttree2 (20). Alphadiversity metrics (Shannon's diversity index), beta diversity metrics (UniFrac (21)) and Principle Coordinate Analysis (PCoA) were estimated using q2-diversity after samples were rarefied (subsampled without replacement) to 69009 sequences per sample. All ASVs were assigned taxonomy using the q2-feature-classifier (22). In brief, a naïve Bayes classifier was trained on the Greengenes 13_8 99\% OTUs reference sequences (23) to assign taxonomy to each ASV. The compositional differences among the groups were determined by a linear discriminant analysis using LEfSe (24) with a threshold of 2.0 on the logarithmic score using Galaxy (https://huttenhower.sph.harvard.edu/galaxy/).

\section{$\underline{\text { Statistical analysis }}$}

Results are shown as mean \pm s.e.m. Visual examination of the data distribution as well as normality testing demonstrated that all variables appeared to be normally distributed. Comparisons and statistical tests were performed as indicated in each figure legend. Briefly, for comparisons of multiple groups over time or with two variables, a two-way analysis of variance (ANOVA) was used and corrected for multiple post hoc comparisons, comparing all groups to each other, all 
groups to a control, or selected groups to each other. For comparisons of multiple groups with only one variable, a one-way ANOVA was performed and corrected for multiple post hoc comparisons. For comparisons of two groups, two-tailed paired or unpaired t tests were used, except where indicated. Statistical analyses were performed in the GraphPad Prism 8 software. The nonparametric Kruskal-Wallis test with a Dunn's post-hoc test was used to assess the difference in median abundance of eukaryotic viral and phage taxa in the Non-IBD, UC and CD cohorts. $P$ values denoted throughout the manuscript highlight biologically relevant comparisons. A $P$ value of less than 0.05 was considered significant, denoted as $* P \leq 0.05, * * P \leq 0.01, * * * P \leq 0.001$, and $* * * * P<0.0001$ for all analyses. FDR correction was performed for all RNA-seq data. 
Fig. S1

A

non-IBD colon

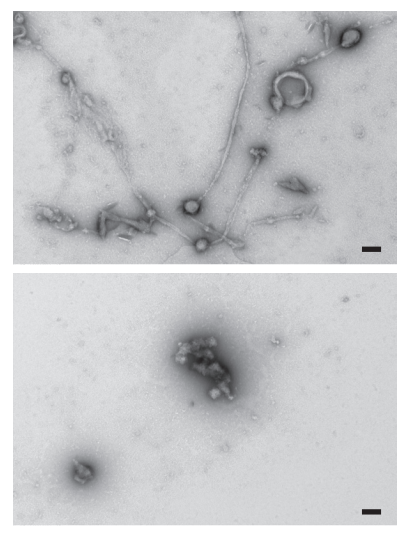

B
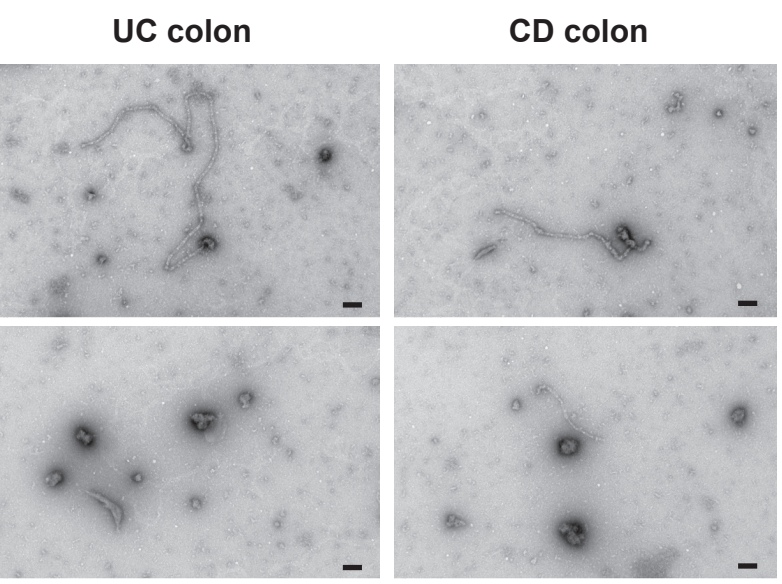

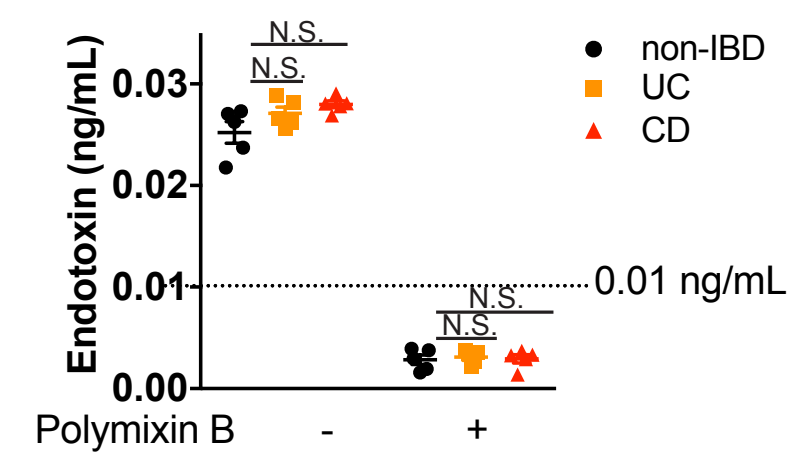

C

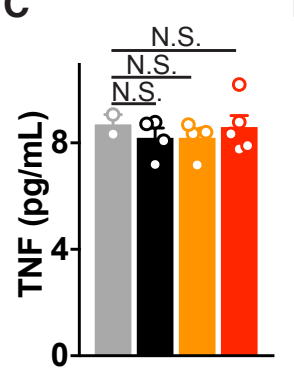

D

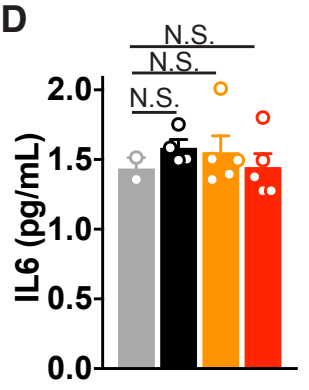

E

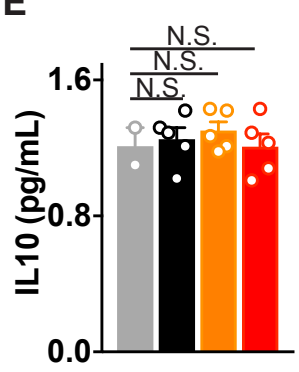

$\mathbf{F}$

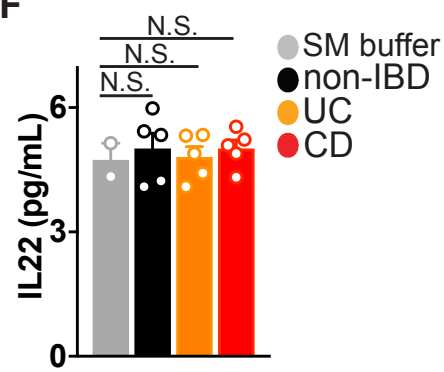

Fig. S1. Isolation of virus-like particles from human colon resections. (A) Representative electron microscopy of virus-like particles (VLPs) isolated from fresh colon resection samples from non-IBD, Ulcerative Colitis (UC) or Crohn's disease (CD) patients, post-surgery using uranyl acetate (scale bar, $100 \mathrm{~nm}$ ). (B) Endotoxin levels in VLPs isolated from colon resections from nonIBD, UC or CD patients as measured by Limulus amebocyte lysate assay. (C) Tumor Necrosis Factor (TNF), (D) Interleukin (IL)-6, (E) IL-10 or (F) IL-22 levels in VLP preparations or buffer controls as measured by ELISA. Data is mean \pm s.e.m. of 3-8 biological replicates, and one-way ANOVA with Tukey's multiple comparison test was used. 
Fig. $\mathbf{S 2}$
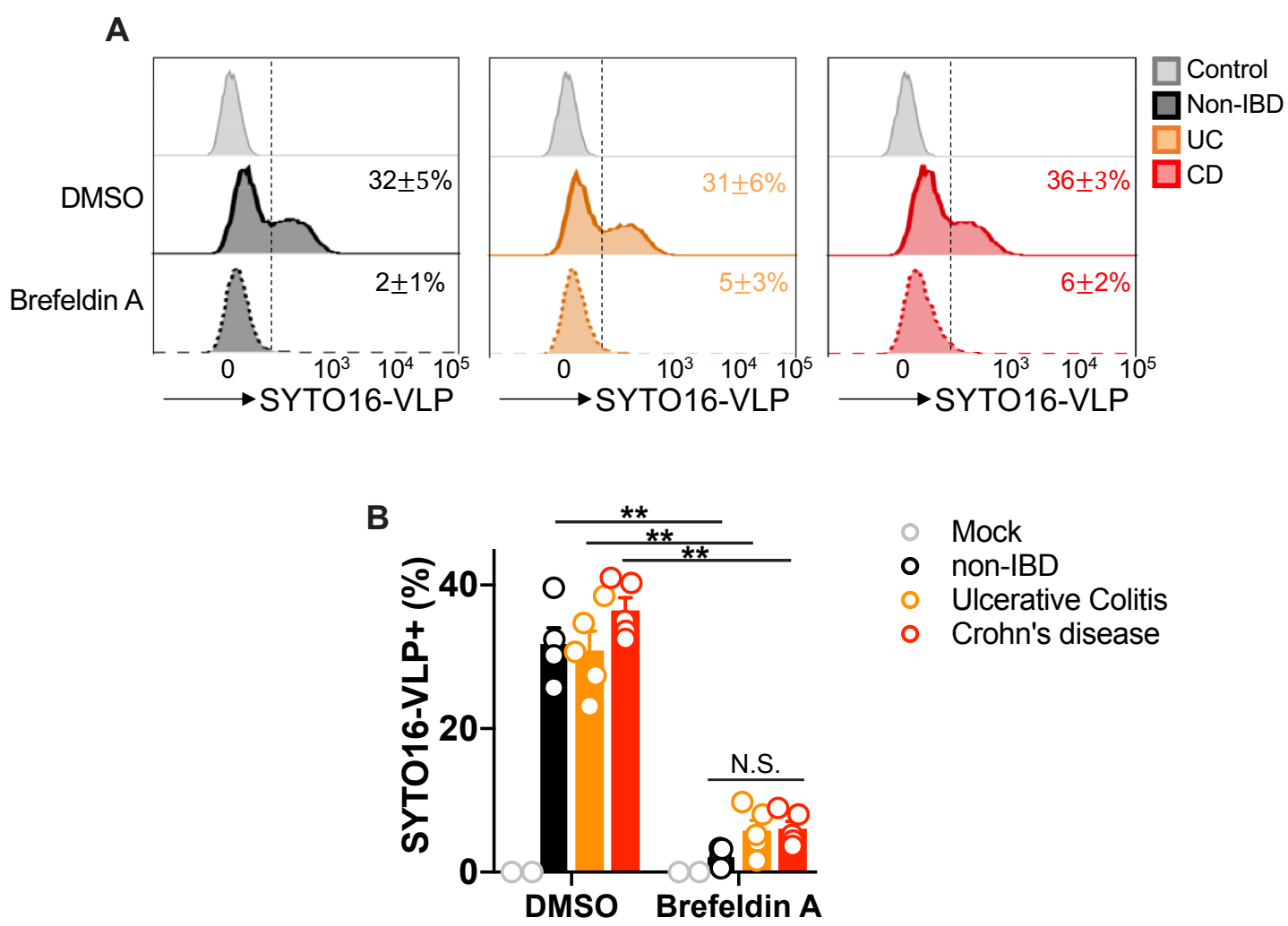

- Mock
- non-IBD
- Ulcerative Colitis
- Crohn's disease

Fig. S2. Internalization of intestine-resident viruses is endocytosis-dependent. (A) Flow cytometric analysis of SYTO-16 labeled virus like particles (VLPs) uptake by primary peripheral blood derived human macrophages 3 hours post incubation with 10 non-IBD, Ulcerative Colitis (UC) or Crohn's disease (CD)-colon resection derived VLPs/cell. Uptake was assessed in the presence of Brefeldin A $(3.0 \mathrm{mg} / \mathrm{mL})$ or DMSO control. (B) Quantification of \% of macrophages that took up SYTO-16 labeled VLPs from 5-8 individuals. Data is mean \pm s.e.m. of 5-8 biological replicates. $* * P<0.01$ and as determined by a one-way ANOVA with Tukey's multiple comparison test. 


\section{Fig. S3}

A

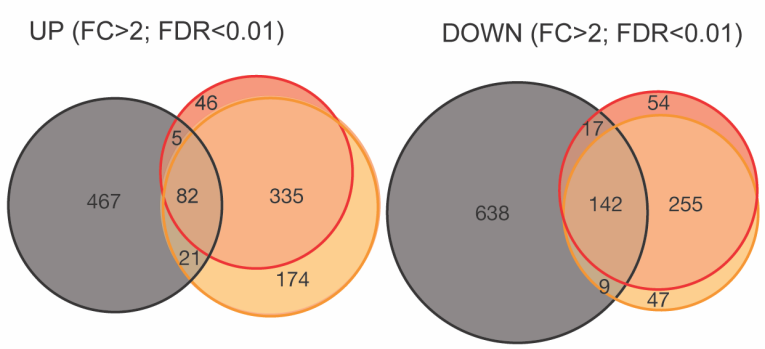

\section{B}
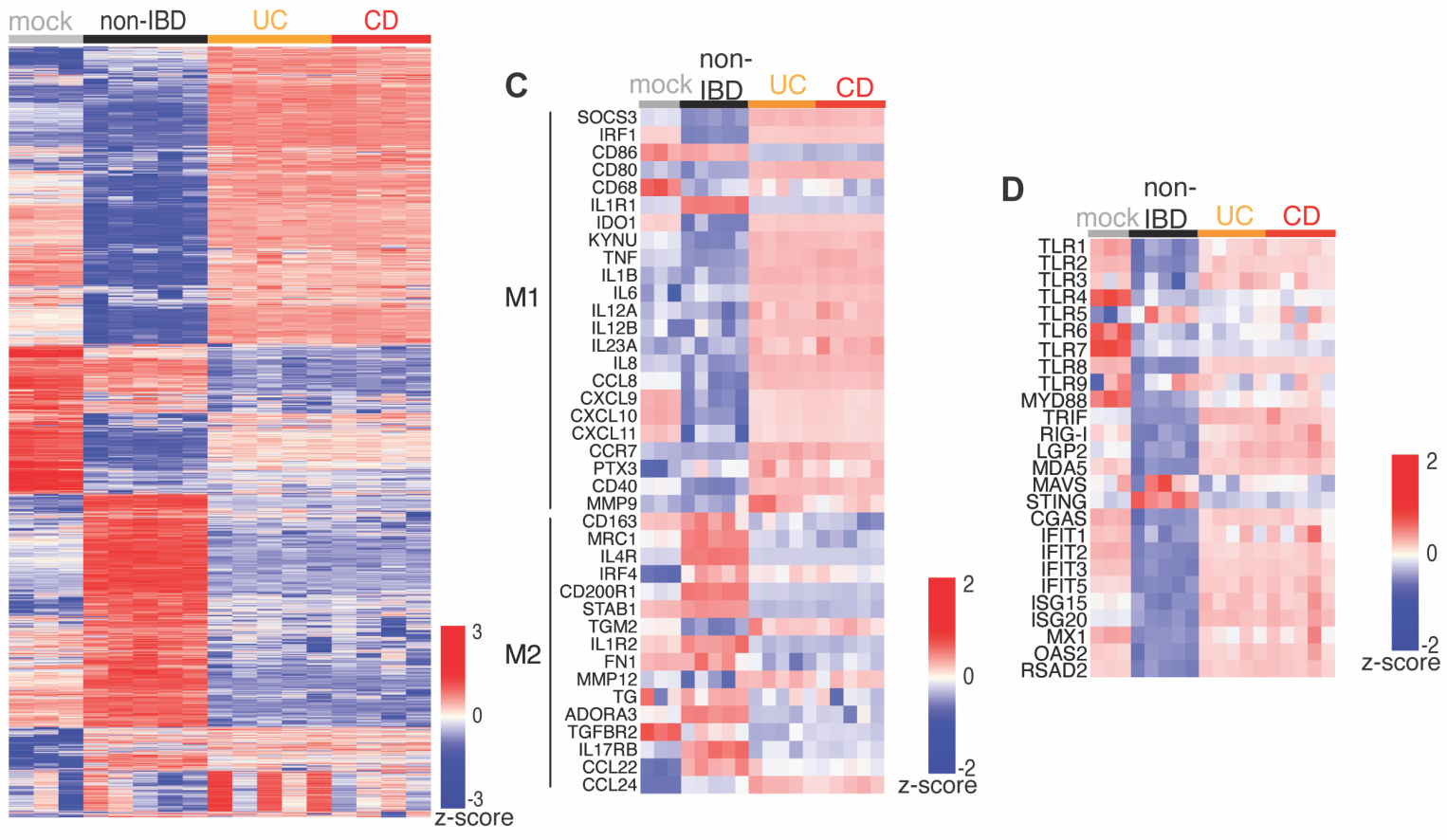

Fig. S3. Divergent macrophage states induced by enteric viruses from non-IBD or IBD patient colons. (A) Venn diagram of genes significantly (Fold Change, FC $>2$; False Discovery Rate, FDR $<0.01$ ) up- or down-regulated by non-IBD, UC or CD VLPs. (B) Hierarchical clustered heatmap of z-score for differentially expressed genes (Fold change $(\mathrm{FC})>2$, false discovery rate $(\mathrm{FDR})<0.05$ ) following exposure of human peripheral blood derived macrophages to mock (SM buffer, $n=3)$ or VLPs from non-IBD $(n=5)$, Ulcerative Colitis $(U C, n=5)$ or Crohn's disease (CD, $\mathrm{n}=5$ ) patient colon resections (10 VLPs/cell, 24h). (C) Heatmap of z-score for select genes defining "M1" pro-inflammatory or "M2" resolving macrophages or (D) Pattern Recognition Receptors (PRRs), signaling adapters and interferon stimulatory genes (ISGs). 
Fig. S4
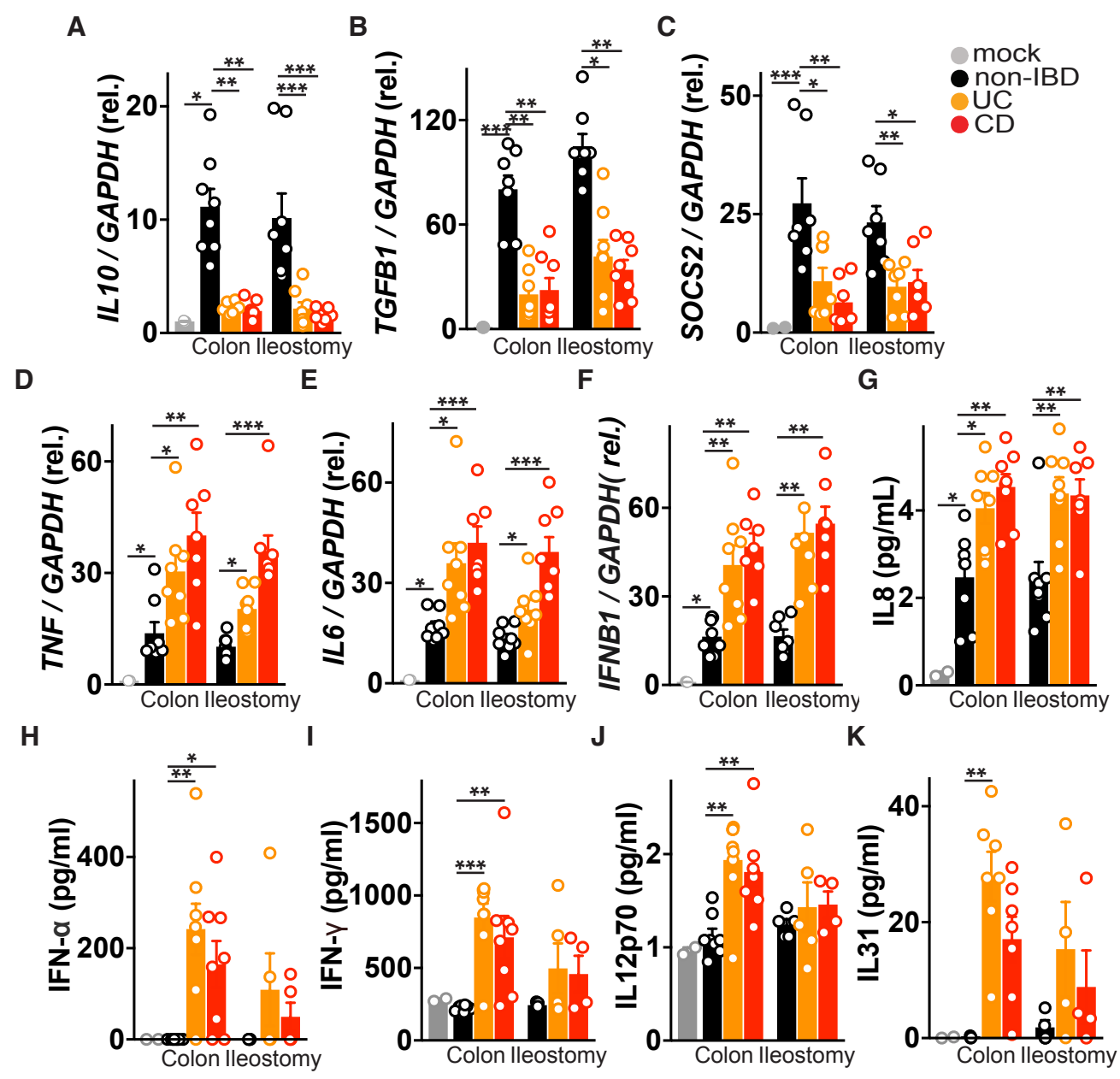

Fig. S4. Enteric viruses from non-IBD patients protect while IBD viromes promote inflammation. Human peripheral blood derived macrophages were delivered virus-like particles (VLPs, 10 VLPs/cell, 24h) isolated from non-IBD controls, Ulcerative Colitis (UC) or Crohn's disease (CD) patient colon resections or ileostomy fluid and (A) IL10, (B) TGFB1, (C) SOCS2, (D) $T N F$, (E) IL6, and (F) IFNBI mRNA levels were measured by qPCR. (G) IL-8, (H) IFN- $\alpha$, (I) IFN- $\gamma,(\mathrm{J})$ IL12p70 and (K) IL-31 production were measured by ELISA. Data is mean \pm s.e.m. of 4-8 biological replicates. ${ }^{*} P<0.05, * * P<0.01, * * * P<0.001$, as determined by a one-way ANOVA with Tukey's multiple comparison test. 


\section{Fig. S5}

A
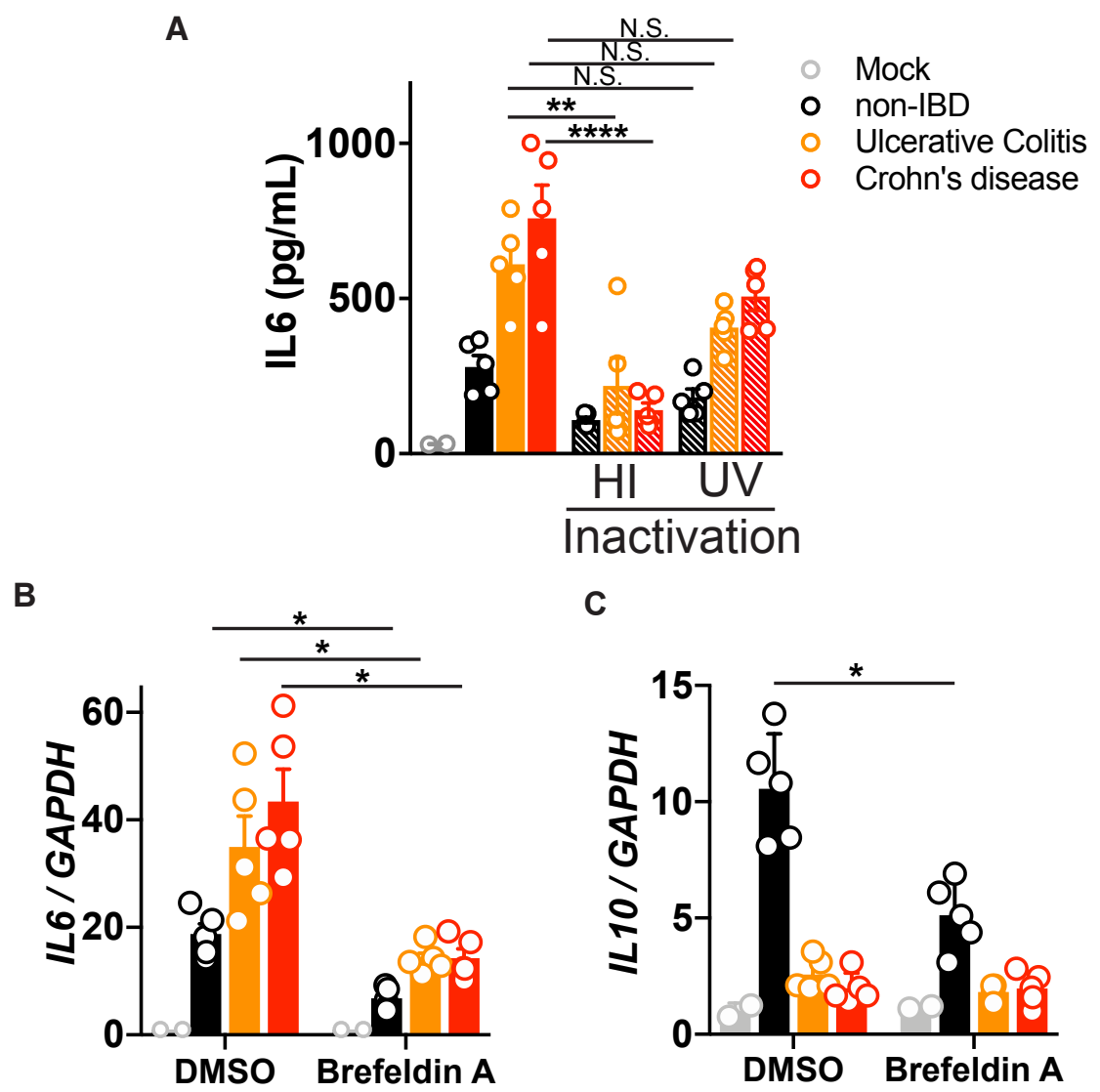

C

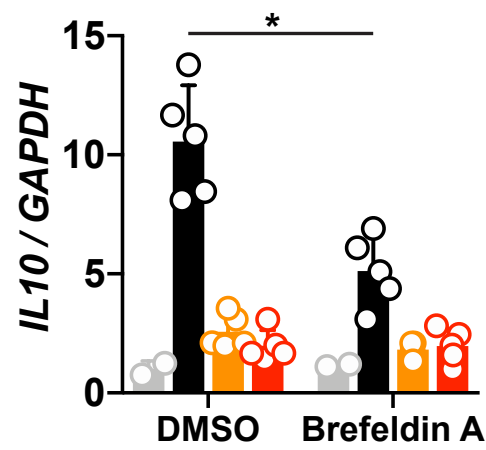

Fig. S5. Requirement of endocytosis but not virus replication for virome immunodulation.

(A) Interleukin (IL)-6 production from primary human peripheral blood-derived macrophages 24 hours following exposure to non-IBD, Ulcerative Colitis (UC) or Crohn's disease (CD) colon resection-derived VLPs, heat-inactivated $\left(60^{\circ} \mathrm{C}\right.$ for $\left.1 \mathrm{~h}\right) \mathrm{VLPs}$, or UV-irradiated (three times 200 $\mathrm{mJ} / \mathrm{cm}^{2}$ for 15 minutes) VLPs, as measured by ELISA. (B) IL-6 or (C) IL-10 levels in human peripheral blood-derived macrophages 24 hours following exposure to non-IBD, UC or CD colon resection-derived VLPs in the presence of Brefeldin A $(3.0 \mathrm{mg} / \mathrm{mL})$ or DMSO control. Data is mean \pm s.e.m. of 5 biological replicates. $* P<0.05, * * P<0.01, * * * P<0.001, * * * * P<0.0001$, as determined by a one-way ANOVA with Tukey's multiple comparison test. 
Fig. S6
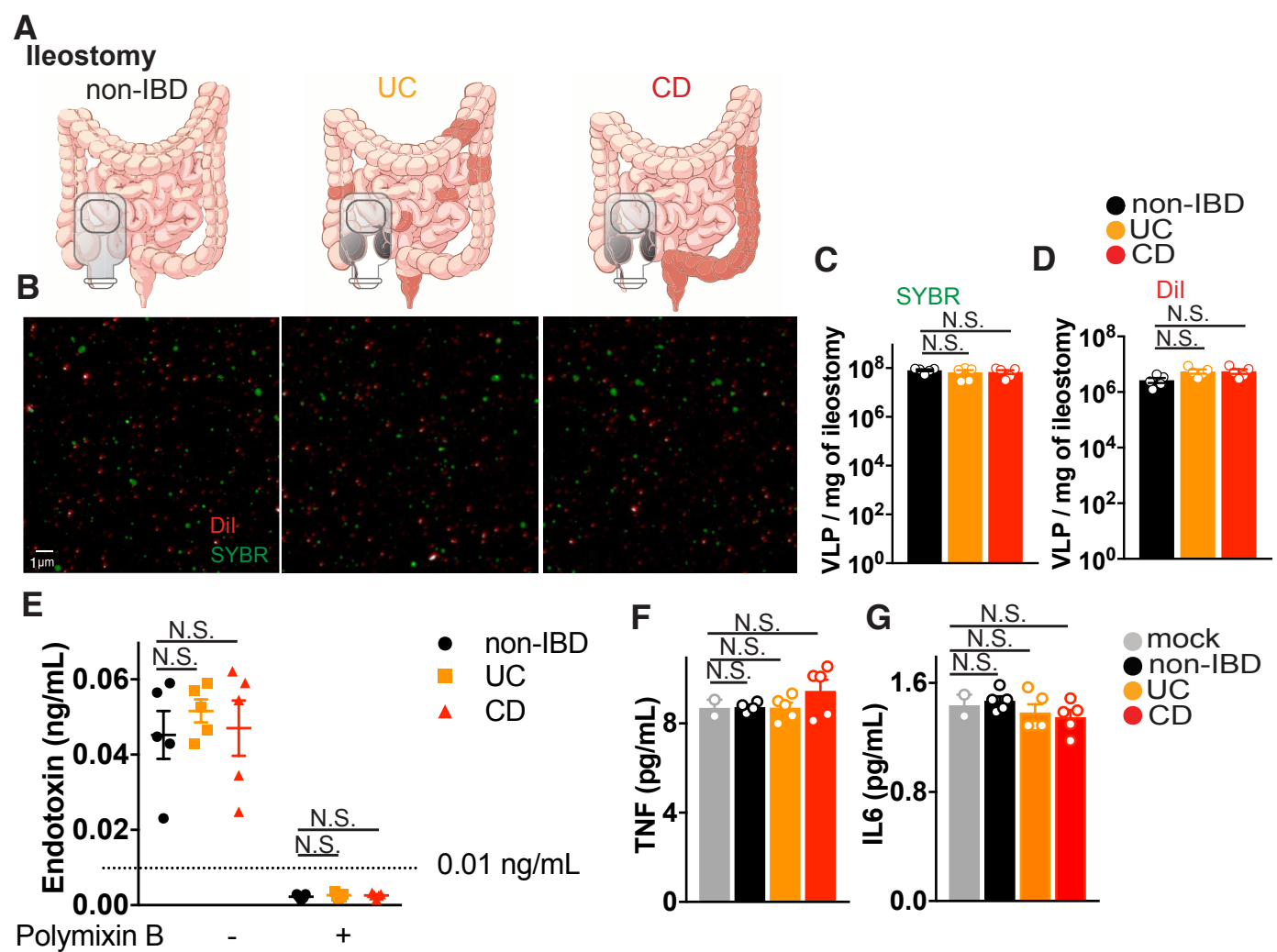

Fig. S6. Isolation of virus-like particles from ileostomy pouch fluid. (A)Virus-like particles (VLPs) were isolated from ileostomy fluid collected from non-IBD, Ulcerative colitis (UC) or Crohn's disease (CD) patients (Table S2). (B) Confocal imaging of VLPs isolated from ileostomy fluid of non-IBD, UC or CD patients (C and D) Quantification of viral nucleic acid using SYBR Gold (C) and phospholipid bilayers using Dialkylcarbocyanine Probe, DiI (D). (E) Endotoxin levels in VLP preps isolated from ileostomy contents of non-IBD, UC or CD patients measured by Limulus amebocyte lysate assay. (F) TNF and (G) IL-6 levels in VLP preps from ileostomy fluids as measured by ELISA. Data is mean \pm s.e.m. of 5-8 biological replicates. Graphs are representative of 2-3 experiments and $n \geq 5$ replicates, and one-way ANOVA with Tukey's multiple comparison test was used. 
Fig. S7

A
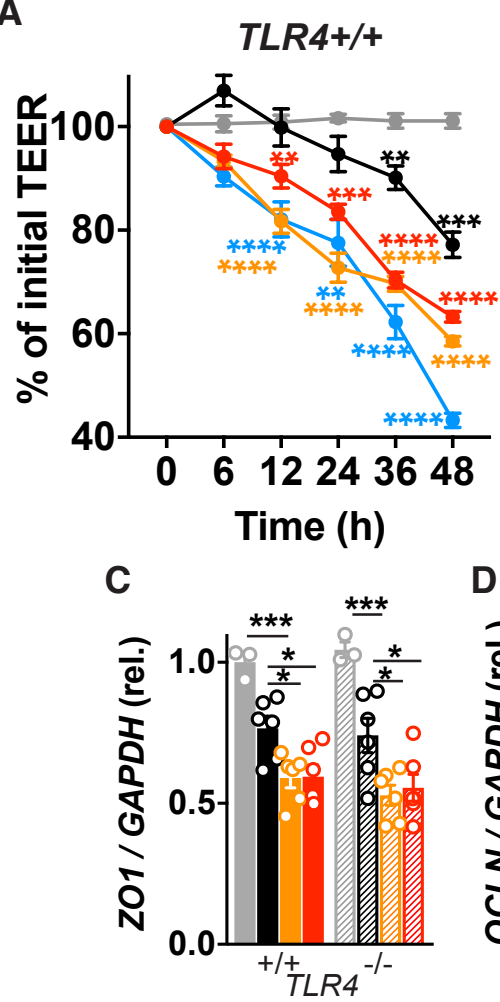

$E$

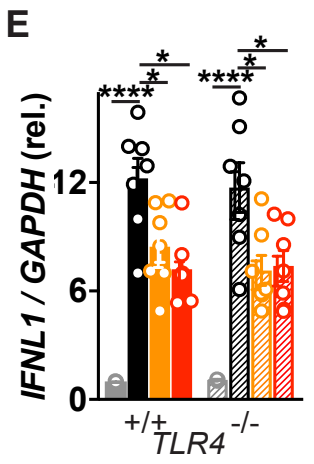

$\mathbf{F}$
B
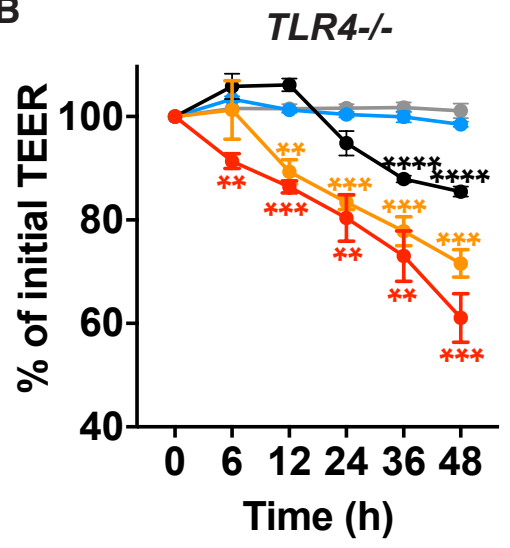

D $\quad$ mock
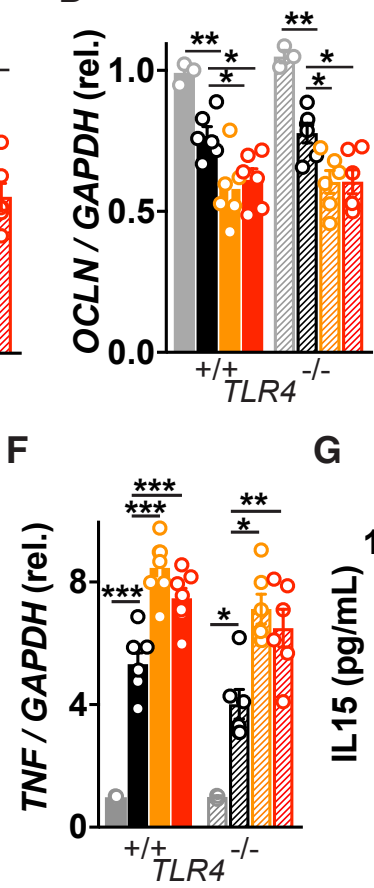

G

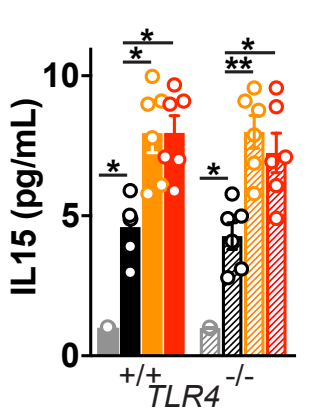

Fig. S7. IBD enteric viruses disrupt intestinal epithelial barrier integrity in a TLR4independent manner. (A) Trans-epithelial electrical resistance (TEER) over time of wild-type or (B) TLR4 ${ }^{-/}$Caco 2 epithelial monolayers following stimulation with LPS $(100 \mathrm{ng} / \mathrm{mL})$ or exposure to SM buffer (mock), non-IBD, Ulcerative colitis (UC) or Crohn's disease (CD) colon resectionderived VLPs (10 VLPs/cell). (C) Zonula occludens (ZO1), (D) Occludin (OCLN), (E) IFNL1, (F) $T N F$ transcript expression or (G) IL-15 levels $48 \mathrm{~h}$ post-incubation of monolayer wild-type or TLR4 knockout Caco2 cells with VLPs isolated from non-IBD, UC and CD colon resections as measured by qPCR or ELISA. Data is mean \pm s.e.m. of $5-8$ biological replicates. ${ }^{*} P<0.05$, $* * P<0.01, * * * P<0.001$, as determined by a one-way ANOVA with Tukey's multiple comparison test. 


\section{Fig. $\mathbf{S 8}$}
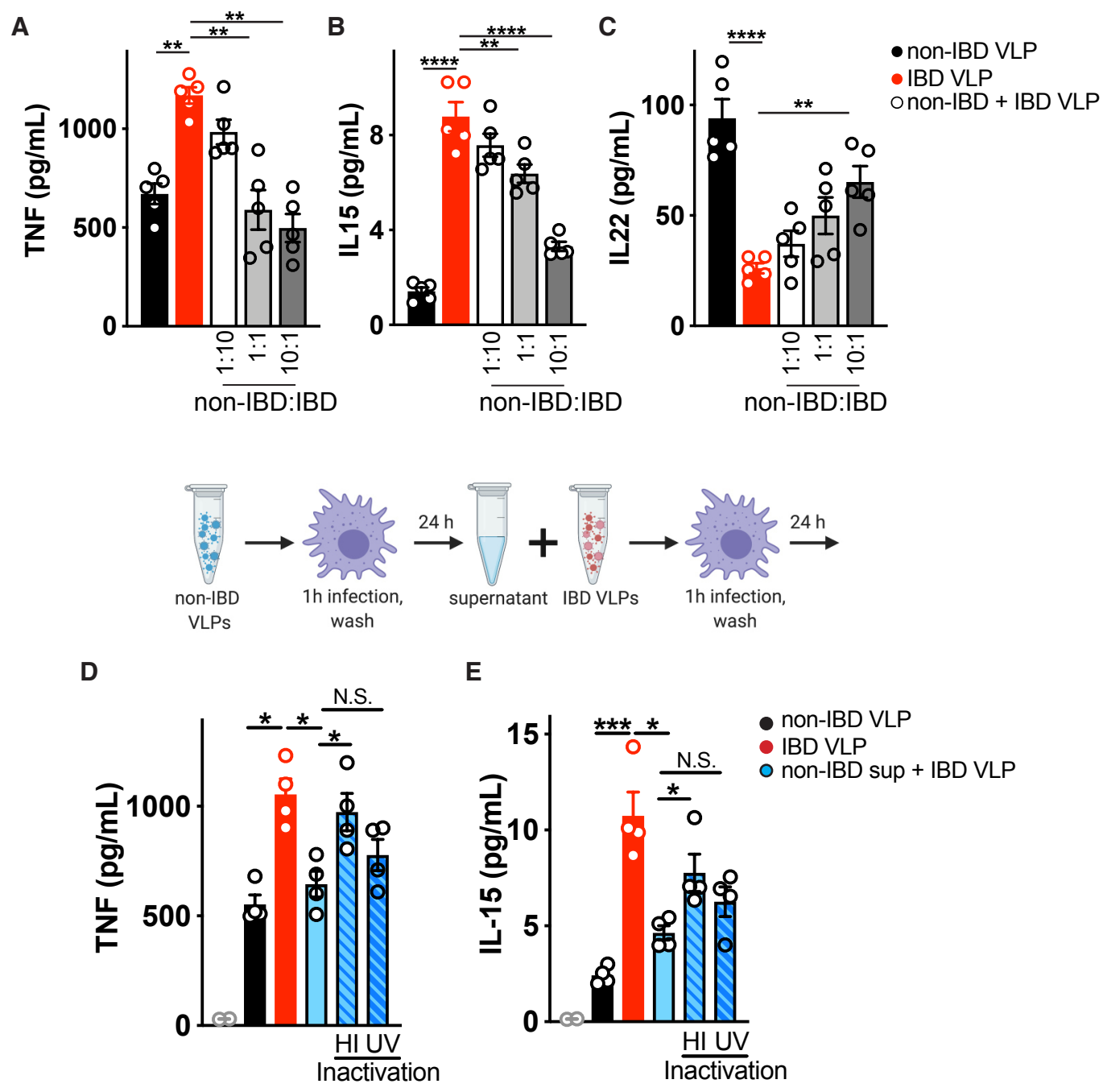

Fig. 8. Suppression of IBD virome-induced inflammation by healthy human enteric viromes. Primary human peripheral blood-derived macrophages were delivered colon resection-derived non-IBD VLPs (10 VLPs/cell), IBD VLPs (10 VLPs/cell) or indicated ratios of non IBD to IBD VLPs and (A) TNF, (B) IL-15, and (C) IL-22 levels were measured at 24h by ELISA. Schematic for incubation of human primary macrophages with IBD VLPs and 24h supernatant from non-IBD VLP exposed macrophages. (D) TNF, and (E) IL-15 levels measured by ELISA following IBD VLP delivery to macrophages with the addition of non-IBD macrophage supernatant that was heat inactivated $\left(95^{\circ} \mathrm{C}\right.$ for $\left.10 \mathrm{~min}\right)$ or $\mathrm{UV}$ crosslinked (three times $200 \mathrm{~mJ} / \mathrm{cm}^{2}$ for 15 minutes) as controls. Data are mean \pm s.e.m. of 5 biological replicates. $* P<0.05, * * P<0.01, * * * P<0.001$, $* * * * P<0.0001$ as determined by one-way ANOVA with Tukey's multiple comparison test. 


\section{Fig. S9}

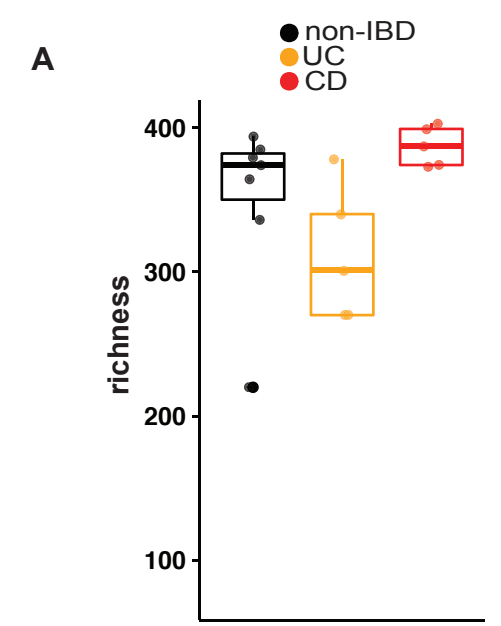

B

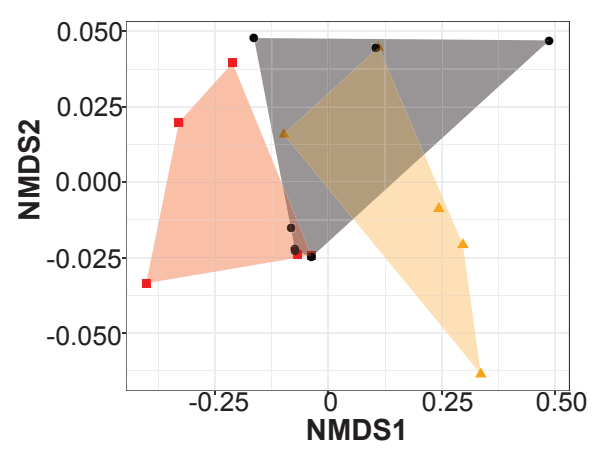

C

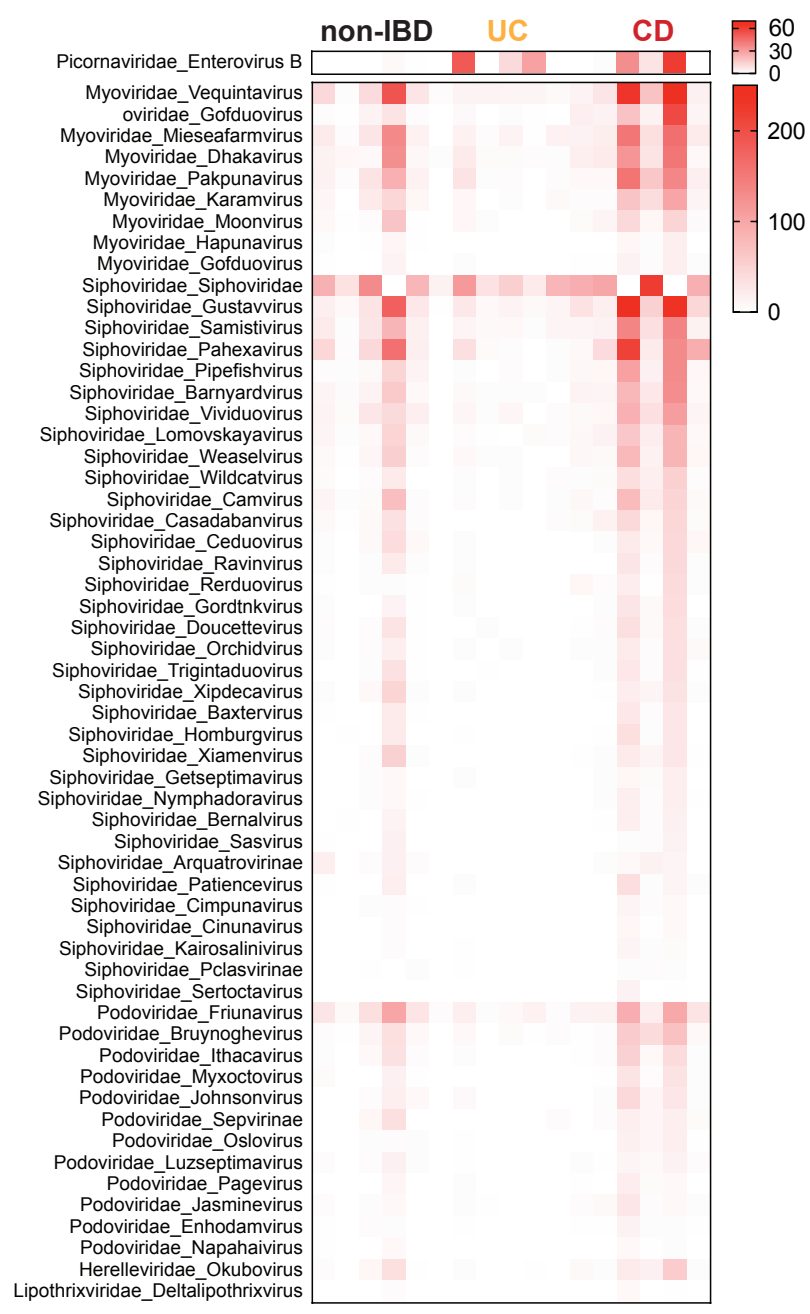

Fig. S9. Characterization of VLPs in colon resections from non-IBD, Ulcerative Colitis and Crohn's disease patients. VLP were assessed using VLP DNA and RNA metagenomic sequence data. Sequences were assigned a eukaryotic viral or phage taxonomic lineage using primary searches against virus sequence databases and subsequently confirmed in secondary searches using reference databases with additional non-viral taxonomic lineages. Specific eukaryotic viral families were selected for subsequent analysis by gating on the alignment length and percent identity for each family that separated the high-quality alignments from potentially spurious alignments (Table S3). (A) VLP species richness was calculated using the spec number function of the Vegan package. Significance was assessed via Kruskal-Wallis test with a Dunn's post-hoc test. (B) Non-metric multidimensional scaling (NMDS) using Bray-Curtis dissimilarity was conducted using the metaMDS function in Vegan and PERMANOVA analysis (permutational multivariate analysis of variance) was performed using the pairwise Adonis package to assess significance. There were no significant differences in beta diversity between the groups. (C) Significantly (P-adjusted $<0.05$, Kruskal-Wallis test with a Dunn's post-hoc correction) differentially abundant eukaryotic and prokaryotic viruses in UC or CD colon resections. Data are the mean of 5-7 biological replicates. 
Fig. S10

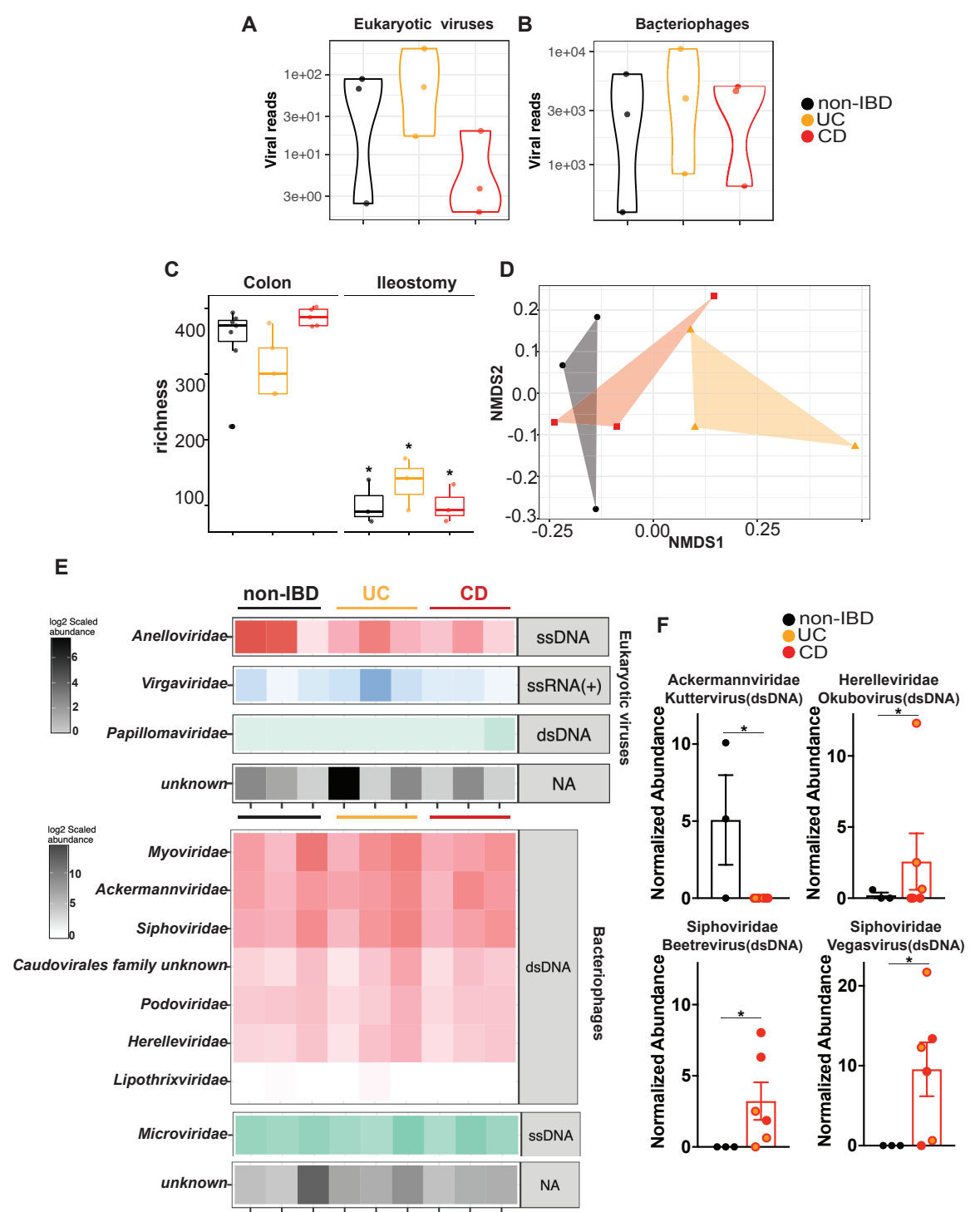

Fig. S10. Detection and characterization of VLPs in ileostomy fluid from non-IBD, Ulcerative Colitis and Crohn's disease patients. (A, B) Violin plots represent the distribution of individual datasets for total reads aligning to eukaryotic viruses or bacteriophages from non-IBD, UC or CD cohorts. (C) Phage VLP richness was assessed using VLP metagenomic sequence data and the spec number function of the Vegan package. Significance was assessed via Kruskal-Wallis test with a Dunn's post-hoc test. (D) NMDS using Bray-Curtis dissimilarity was conducted using the metaMDS function in Vegan and PERMANOVA analysis was performed using the pairwise Adonis package to assess significance. There were no significant differences in beta diversity between the groups. (E) Taxonomic assignments of VLP sequences with high quality alignments in non-IBD, UC or CD ileostomy samples. The families are grouped by their Baltimore Classification and ranked by relative abundance in the dataset. (F) Significantly differentially abundant genera of bacteriophages in ileostomy samples from non-IBD versus IBD cohorts. Data are the mean of 5-7 biological replicates. ${ }^{*} P<0.05$, Kruskal-Wallis test with a Dunn's post-hoc test. 

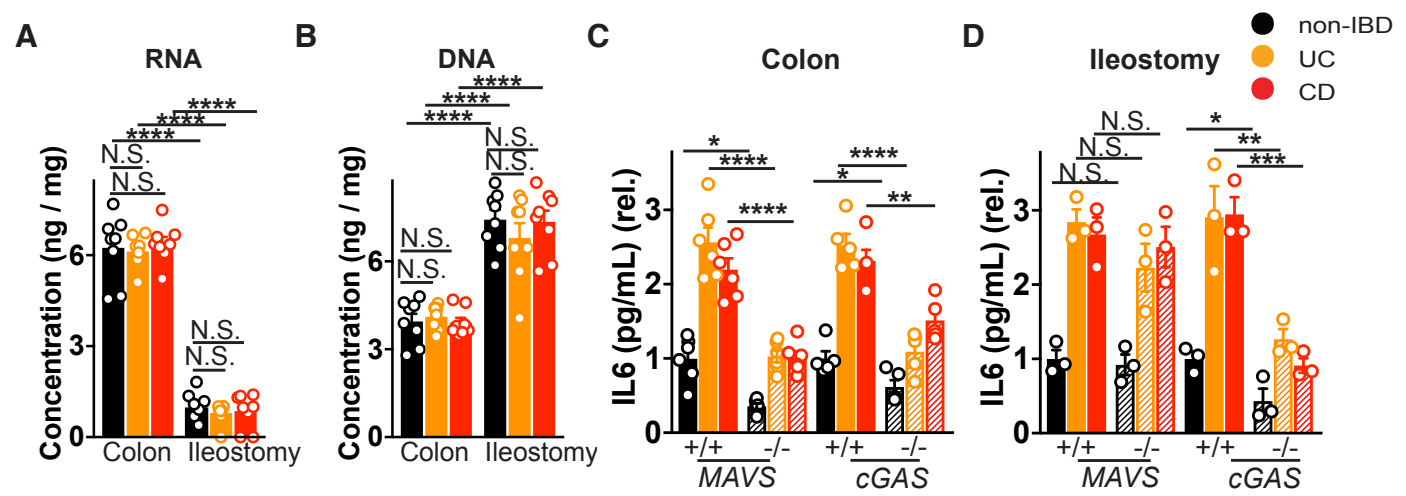

Fig. S11. Differential host receptor requirement for immune response to VLPs isolated from colon or ileostomy fluid. Concentration of (A) RNA, and (B) DNA isolated from non-IBD, Ulcerative colitis (UC) or Crohn's disease (CD) colon resections or ileostomy fluid derived VLPs. (C) IL-6 levels following exposure of bone marrow derived macrophages (BMDMs) from wildtype, $\mathrm{MAVS}^{-/-}$or $\mathrm{cGAS}^{-/-}$mice to VLPs $(10 \mathrm{VLPs} / \mathrm{cell}, 24 \mathrm{~h})$ derived from $(\mathrm{C})$ non-IBD, UC or CD colon resections or (D) ileostomy fluid as measured by ELISA. Data is mean \pm s.e.m. of 3-6 biological replicates. $* P<0.05, * * P<0.01, * * * P<0.001$, **** $P<0.0001$, as determined by aneway ANOVA with Tukey's multiple comparison test. 
Fig. S12

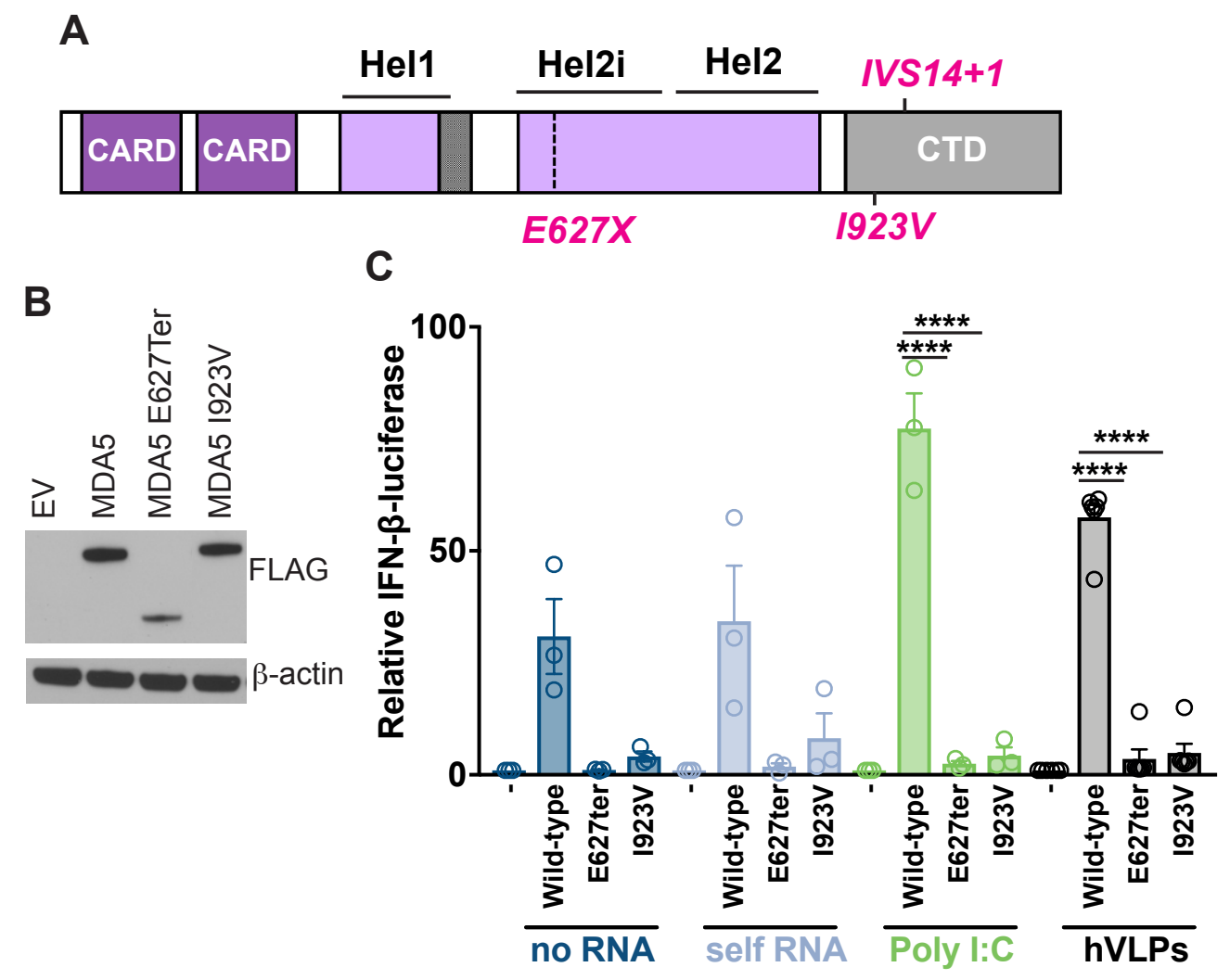

Fig. S12. IBD-risk loss-of-function MDA5 exhibits dampened responses to the human enteric virome. (A) Schematic of human MDA5 and mapped IBD-associated MDA5 variants rs35744605/E627X, rs35667974/I923V and rs35732034/IVS14+1. (B) Western Blot of ectopically expressed, FLAG-tagged MDA5 in HEK293T cells. (C) Relative activation of IFN $\beta$ luciferase reporter in HEK293T cells transfected with Empty vector (EV), FLAG-tagged WT MDA5, MDA5E627Ter or MDA5I923V and transfected with $1 \mu \mathrm{g} / \mathrm{mL}$ RNA isolated from the cytoplasm of HEK293T cells (self RNA), poly I:C or RNA isolated from human colon resection virus like particles (VLPs) for 24 hours. 
Fig. S13

A

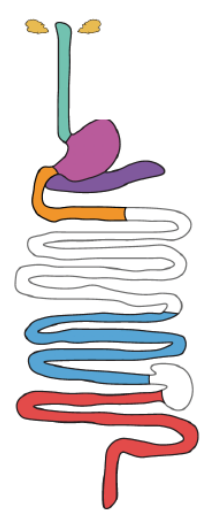

B

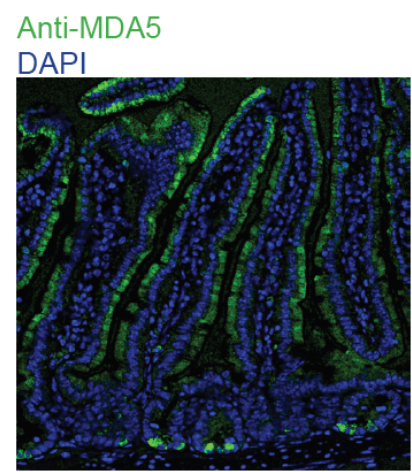

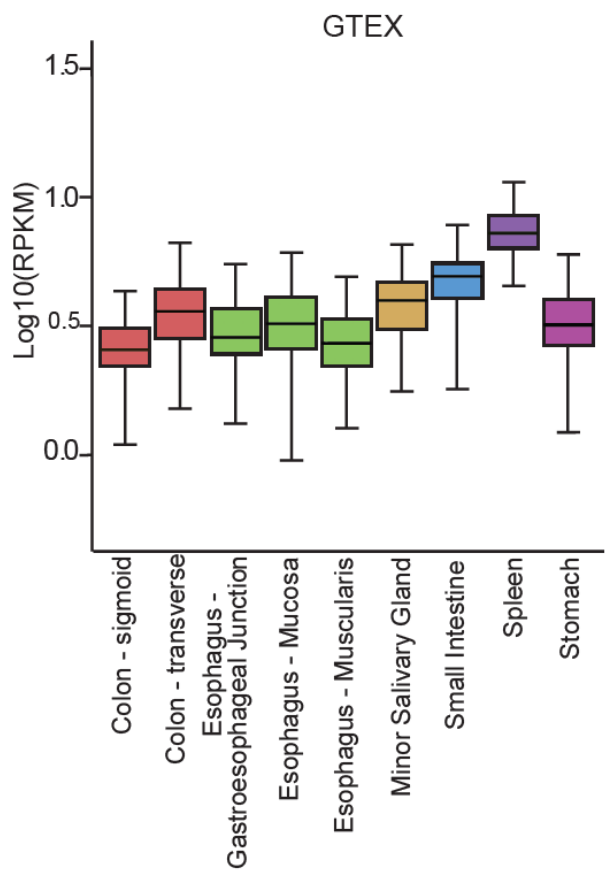

C

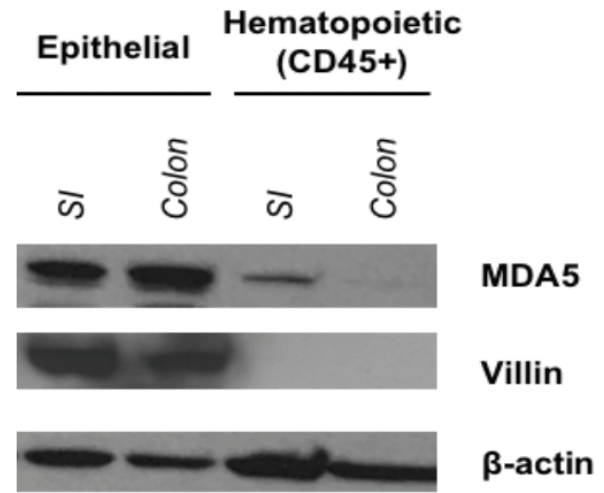

Fig. S13. Virus receptor MDA5 is preferentially expressed in intestinal epithelium. (A) IFIH1 (MDA5) mRNA levels across several gastrointestinal-related human tissues and spleen were retrieved from Genotype-Tissue Expression (GTEX) Portal (Broad Institute) (B) Confocal microscopy of MDA-5 (green) or nuclei (DAPI, blue) in mouse small intestine. (C) Immunoblot of MDA5 in epithelial cells or CD45+ hematopoietic cells sorted from mouse small intestine (SI) or colon. 


\section{Fig. S14}

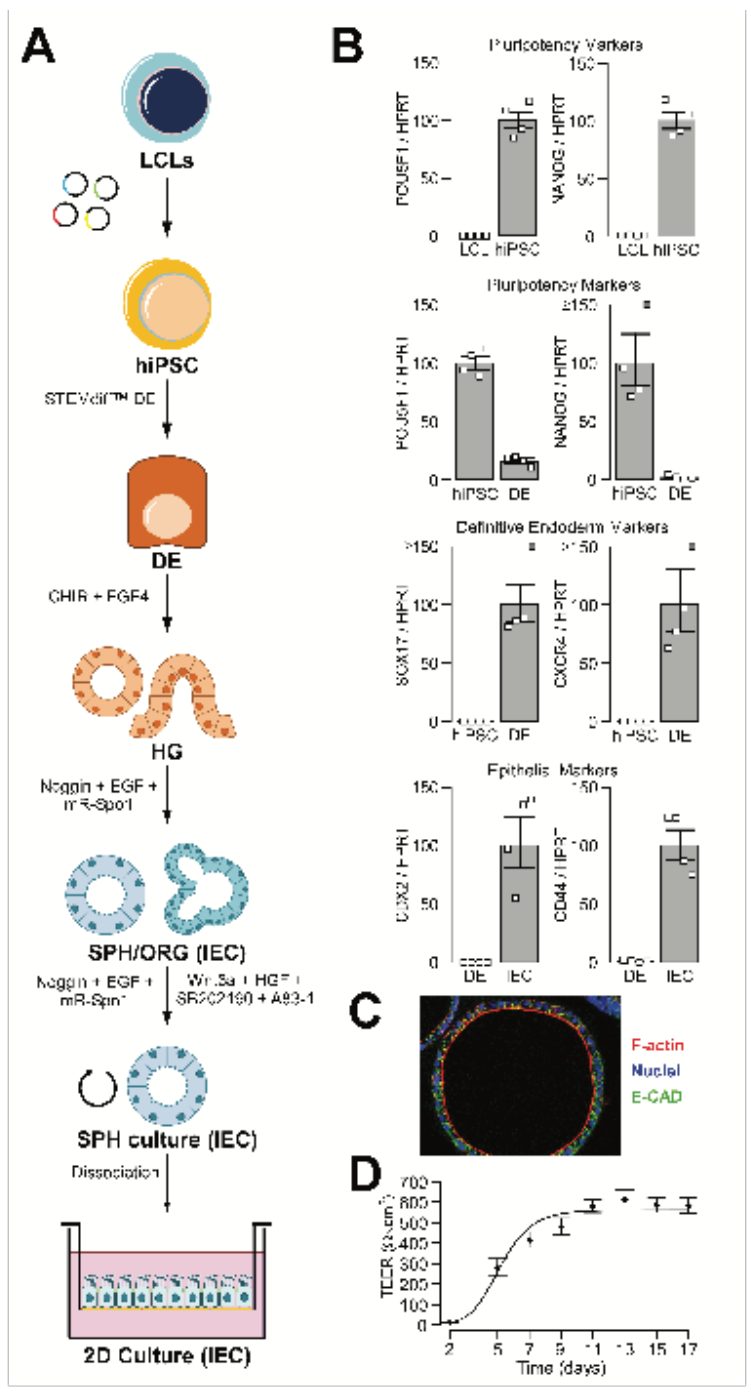

Fig. S14. Generation of hiPSC-derived human intestinal epithelial cells. (A) Schematic illustrating the different steps involved in the generation of hiPSC-derived 2D epithelial cultures from LCLs. (B) Progression through the different steps of the differentiation protocol is measured via the expression of specific markers by qPCR: (1) conversion of LCLs to hiPSC is shown by the increased expression of pluripotency markers such as POU5F1 and NANOG; (2) differentiation of hiPSC to DE is confirmed through the loss of these pluripotency markers as well as with the gain of DE-specific markers such as SOX17 and CXCR4; (3) the generation of IEC cultures is validated by the expression of intestinal-specific markers such as CDX2 and CD44. (C) Epithelial cultures are also evaluated through confocal immunofluorescence imaging to confirm the formation of epithelial-like polarized structure (spheroids are shown; for IF of 2D epithelial monolayer see Fig 10): nuclei (DAPI, blue), F-actin (phalloidin, red), and E-cadherin (green). (D) Barrier integrity of epithelial monolayers is assessed by measurement of transepithelial electric resistance (TEER). 
Fig. S15

A
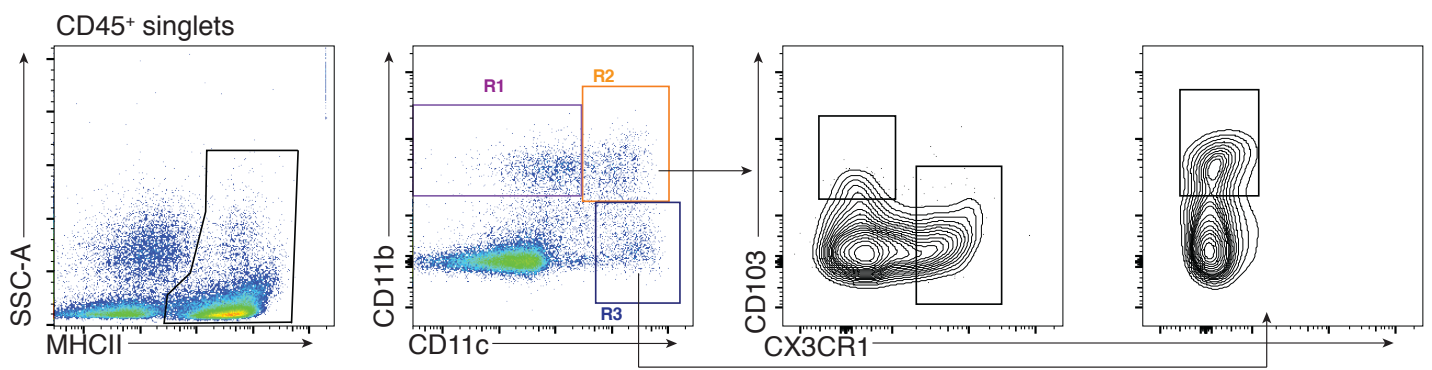

B
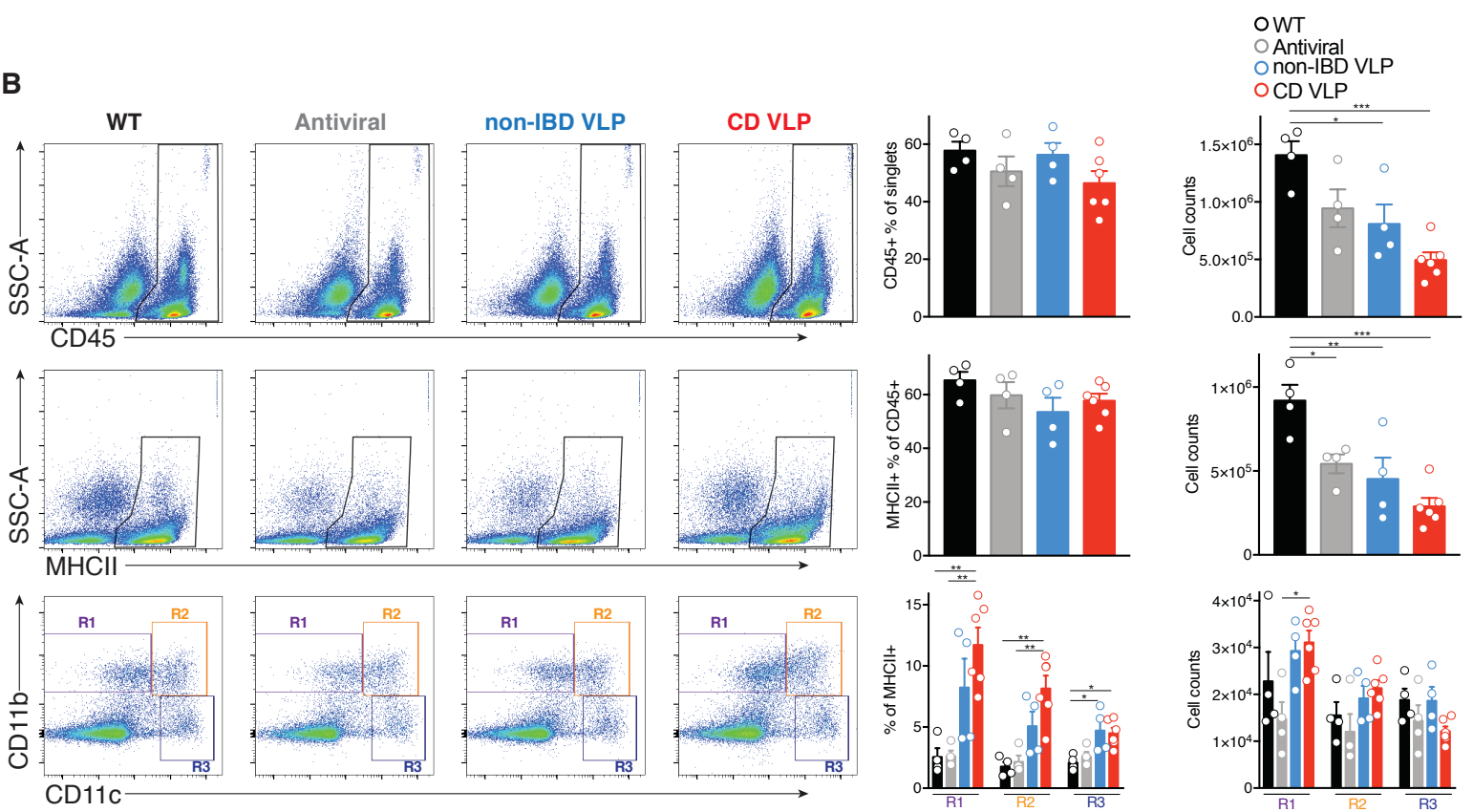

Fig. S15. Quantification of mononuclear phagocytes in colonic lamina propria of humanized virome mice. $\mathrm{C} 57 \mathrm{BL} / 6$ mice were depleted of their enteric virome by an antiviral cocktail (Acyclovir 20mg/kg, Lamivudine 10mg/kg, Ribavirin 30mg/kg, Oseltamivir 10mg/kg, gray, daily gavage for 10 days) and reconstituted with VLPs isolated from colonic resections of 4 non-IBD or 6 Crohn's disease (CD) patients. (A) Colonic lamina propria were isolated as described in the methods section and analyzed using the described gating strategy. Cells were first gated on CD45+/MHCII+ live singlets and CD11b+CD11c- (R1), CD11b+CD11c+ (R2) and CD11bCD11c+ (R3) mononuclear phagocytes were examined for expression of CX3CR1 or CD103. (B) Representative flow cytometry plots and quantification of frequency and the total count for the given immune cell subsets, including total $\mathrm{CD} 45+$ singlets (top), CD45+/MHCII + singlets (middle), and $\mathrm{CD} 11 \mathrm{~b}$ or $\mathrm{CD} 11 \mathrm{c}$ expressing CD45+/MHCII+ singlets (bottom). Each circle represents the cells from one mouse. Data is mean \pm s.e.m. of 4-6 mice per group. ${ }^{*} P<0.05$, $* * P<0.01$ and $* * * P<0.001$ as determined by a one-way ANOVA with Tukey's multiple comparison test. 
Fig. S16

A

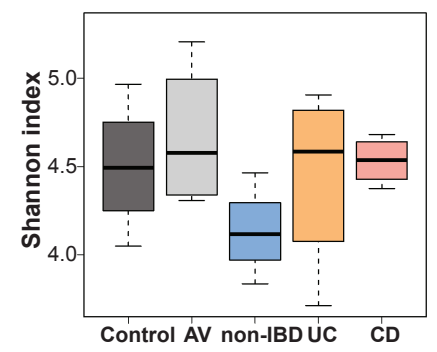

C

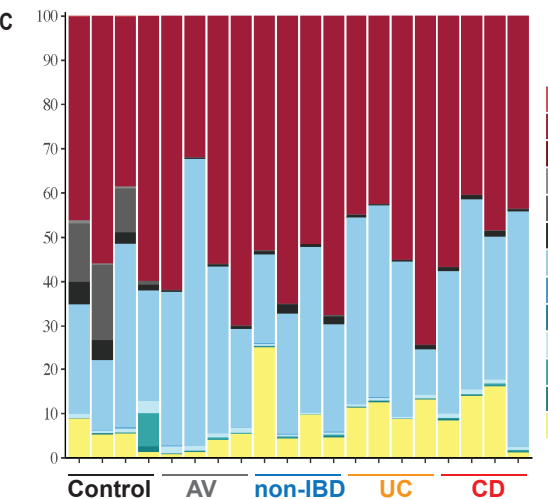

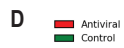

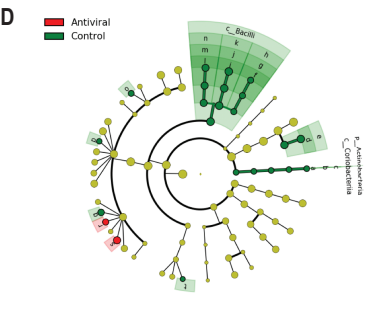

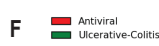
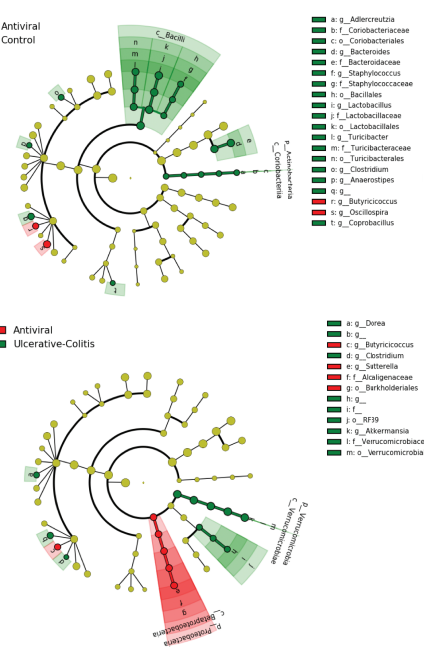

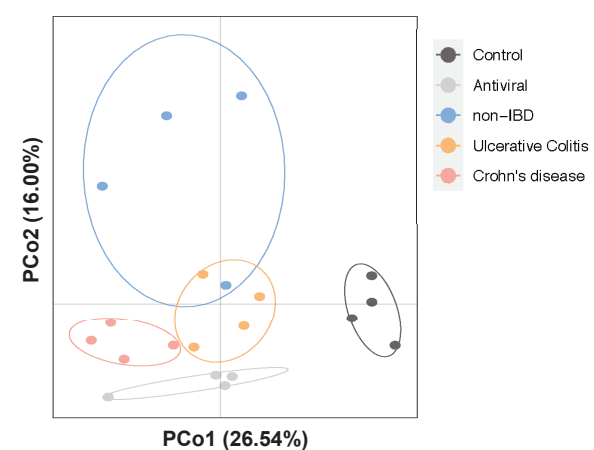

k_Bacteria;

- _Bacteria,p__Bacteroid

k_Bacteria;P_Cyanobacteria;c_CChloroplast;o_Streptophyta

k_Bacteria;p_Firmicutes;c_Bacilli;o__Lactobacillales

k_Bacteria;p_Firmicutes;c_Bacilli;o_Turicibacterales

k_Bacteria;p_Firmicutes;c_Clostridia;o_Clostridiales

k_Bacteria;p_Firmicutes;c_Erysipelotrichi;o_Erysipelotrichales

k_Bacteria;p_Proteobacteria;c_Alphaproteobacteria;o_Rhizobiales

k_Bacteria; ;_Proteobacteria; __Betaproteobacteria;___Burkholderiales

k_Bacteria;p_Tenericutes;c_Mollicutes;o_Anacroplasmatales

k_Bacteria;p_Tenericutes;c_Mollicutes;o_RF39

k_Bacteria;p_Verrucomicrobia;c_Verrucomicrobiae;o_Verrucomicrobiale

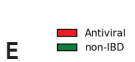
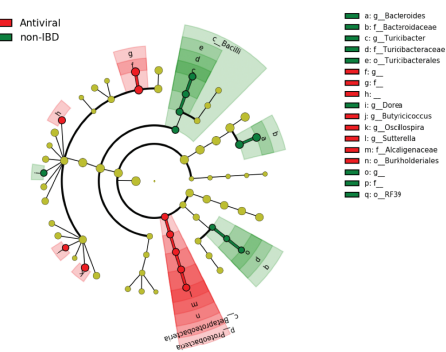

G
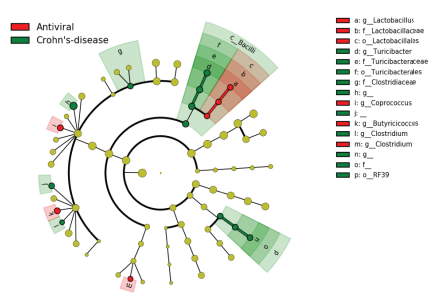

Fig. S16. Abundance and composition of C57BL/6 murine bacterial communities following administration of human enteric viromes. Murine fecal bacterial communities before and after antiviral cocktail treatment and following gavage administration of healthy, Ulcerative colitis (UC) or Crohn's disease (CD) human colon resection-derived viromes were determined using 16S rRNA sequencing. (A) Diversity of bacterial communities as determined by Shannon Index. (B) Principle Coordinate Analysis (PCoA), P $<0.05$, PERMANOVA on unweighted UniFrac distance. (C) Distribution of operational taxonomic units (key) in feces; each operational taxonomic unit (selected at a sequence identity of 99\%; QIIME software) is presented as relative abundance in each sample. (D) The compositional differences between control and antiviral treated mice, (E) antiviral treated compared to non-IBD virome humanized mice, $(\mathrm{F})$ antiviral treated compared to UC virome humanized mice and $(\mathrm{G})$ antiviral treated compared to $\mathrm{CD}$ virome humanized mice were determined by a linear discriminant analysis using LEfSe with a threshold of 2.0 on the logarithmic score using Galaxy (https://huttenhower.sph.harvard.edu/galaxy/). 
Table S1. Demographic Data of Participants in the Study, Colon Resections.

\begin{tabular}{|c|c|c|c|c|c|c|}
\hline $\begin{array}{c}\text { Sample } \\
\#\end{array}$ & Diagnosis & Pathology & Gender & $\begin{array}{l}\text { Patient } \\
\text { Age } \\
\text { (year) }\end{array}$ & $\begin{array}{l}\text { Duration } \\
\text { of } \\
\text { disease } \\
\text { (year) }\end{array}$ & Medications \\
\hline T1 & $\begin{array}{l}\text { Non-IBD, Colon } \\
\text { cancer, } \\
\text { Adenocarcinoma }\end{array}$ & $\begin{array}{c}\text { Non- } \\
\text { inflamed }\end{array}$ & $\mathrm{F}$ & 63 & 15 & $\begin{array}{l}\text { Prednisone, } \\
\text { Metronidazole }\end{array}$ \\
\hline $\mathrm{T} 2$ & $\begin{array}{c}\text { Non-IBD, } \\
\text { Diverticulitis }\end{array}$ & $\begin{array}{c}\text { Non- } \\
\text { inflamed }\end{array}$ & M & 51 & 11 & $\begin{array}{l}\text { Prednisone, } \\
\text { Ciprofloxacin } \\
\text { Levaquin } \\
\text { Budesonide }\end{array}$ \\
\hline T3 & $\begin{array}{l}\text { Non-IBD, Colon } \\
\text { cancer, } \\
\text { Adenocarcinoma }\end{array}$ & $\begin{array}{c}\text { Non- } \\
\text { inflamed }\end{array}$ & $F$ & 67 & 10 & $\begin{array}{c}\text { Prednisone, } \\
\text { Ciprofloxacin } \\
\text { Levaquin }\end{array}$ \\
\hline $\mathrm{T} 4$ & $\begin{array}{c}\text { Non-IBD, } \\
\text { Familial } \\
\text { Adenomatous } \\
\text { Polyposis }\end{array}$ & $\begin{array}{c}\text { Non- } \\
\text { inflamed }\end{array}$ & M & 23 & 1 & $\begin{array}{l}\text { Prednisone, } \\
\text { Metronidazole }\end{array}$ \\
\hline T5 & $\begin{array}{c}\text { Non-IBD, } \\
\text { Familial } \\
\text { Adenomatous } \\
\text { Polyposis }\end{array}$ & $\begin{array}{c}\text { Non- } \\
\text { inflamed }\end{array}$ & M & 43 & 7 & $\begin{array}{l}\text { Ciprofloxacin } \\
\text { Levaquin }\end{array}$ \\
\hline T6 & $\begin{array}{l}\text { Non-IBD, } \\
\text { Diverticulitis }\end{array}$ & $\begin{array}{c}\text { Non- } \\
\text { inflamed }\end{array}$ & M & 53 & 21 & \\
\hline T7 & $\begin{array}{l}\text { Non-IBD, } \\
\text { Diverticulitis }\end{array}$ & $\begin{array}{l}\text { Non- } \\
\text { inflamed }\end{array}$ & $F$ & 76 & 25 & $\begin{array}{l}\text { Prednisone, } \\
\text { Metronidazole }\end{array}$ \\
\hline T8 & $\begin{array}{c}\text { Non-IBD, } \\
\text { Diverticulitis }\end{array}$ & $\begin{array}{l}\text { Non- } \\
\text { inflamed }\end{array}$ & M & 57 & 15 & \\
\hline T9 & Ulcerative colitis & Inflamed & $\mathrm{F}$ & 38 & 4 & \\
\hline T10 & Ulcerative colitis & Inflamed & $F$ & 63 & - & $\begin{array}{l}\text { Prednisone, } \\
\text { Azathioprine, } \\
\text { Metronidazole }\end{array}$ \\
\hline T11 & Ulcerative colitis & Inflamed & M & 51 & 10 & \\
\hline T12 & Ulcerative colitis & Inflamed & $\mathrm{F}$ & 42 & 11 & $\begin{array}{l}\text { Prednisone, } \\
\text { Azathioprine, } \\
\text { Metronidazole }\end{array}$ \\
\hline T13 & Ulcerative colitis & Inflamed & M & 32 & - & \\
\hline T14 & Ulcerative colitis & Inflamed & $M$ & 24 & 1 & $\begin{array}{l}\text { Prednisone, } \\
\text { Azathioprine, }\end{array}$ \\
\hline
\end{tabular}




\begin{tabular}{|c|c|c|c|c|c|c|}
\hline T15 & Ulcerative colitis & Inflamed & M & 68 & 11 & $\begin{array}{l}\text { Prednisone, } \\
\text { Azathioprine, } \\
\text { Metronidazole }\end{array}$ \\
\hline T16 & Ulcerative colitis & Inflamed & M & 46 & 8 & \\
\hline $\mathrm{T} 17$ & Crohn's disease & Inflamed & $\mathrm{M}$ & 33 & 4 & $\begin{array}{l}\text { Prednisone, } \\
\text { Azathioprine, } \\
\text { Metronidazole }\end{array}$ \\
\hline T18 & Crohn's disease & Inflamed & $\mathrm{F}$ & 28 & 3 & \\
\hline T19 & Crohn's disease & Inflamed & $\mathrm{F}$ & 51 & 9 & $\begin{array}{c}\text { Prednisone, } \\
\text { Azathioprine, } \\
\text { Ciprofloxacin } \\
\text { Levaquin }\end{array}$ \\
\hline T20 & Crohn's disease & Inflamed & $\mathrm{M}$ & 62 & 28 & \\
\hline T21 & Crohn's disease & Inflamed & $\mathrm{F}$ & 28 & 1 & $\begin{array}{l}\text { Prednisone, } \\
\text { Azathioprine, } \\
\text { Metronidazole }\end{array}$ \\
\hline T22 & Crohn's disease & Inflamed & M & 48 & 26 & $\begin{array}{c}\text { Prednisone, } \\
\text { Azathioprine, } \\
\text { Ciprofloxacin } \\
\text { Levaquin }\end{array}$ \\
\hline T23 & Crohn's disease & Inflamed & $\mathrm{M}$ & 52 & 7 & \\
\hline T24 & Crohn's disease & Inflamed & $\mathrm{F}$ & 63 & & \\
\hline
\end{tabular}


Table S2. Demographic Data of Participants in the Study, Ileotomy Samples

\begin{tabular}{|c|c|c|c|c|}
\hline Sample\# & Diagnosis & Gender & Age (year) & Experiments \\
\hline 11 & $\begin{array}{l}\text { Non-IBD, Colonic stricture, } \\
\text { Diverticular disease }\end{array}$ & $M$ & 56 & Figure $1 \mathrm{M}-\mathrm{N}$ \\
\hline 12 & $\begin{array}{l}\text { Non-IBD, Colon cancer } \\
\text { Adenocarcinoma }\end{array}$ & $\mathrm{F}$ & 76 & Figure $1 \mathrm{M}-\mathrm{N}$ \\
\hline 13 & Non-IBD, Colonic stricture & M & 59 & Figure $1 \mathrm{M}-\mathrm{N}$ \\
\hline 14 & $\begin{array}{l}\text { Non-IBD, Colon cancer } \\
\text { Adenocarcinoma }\end{array}$ & M & 61 & Figure $1 \mathrm{M}-\mathrm{N}$ \\
\hline 15 & Non-IBD, Collagenous colitis & M & 71 & Figure $1 \mathrm{M}-\mathrm{N}$ \\
\hline 16 & Non-IBD, Motility disorder & $\mathrm{F}$ & 68 & Figure $1 \mathrm{M}-\mathrm{N}$ \\
\hline 17 & $\begin{array}{l}\text { Non-IBD, Familial Adenomatous } \\
\text { Polyposis }\end{array}$ & M & 72 & Figure $1 \mathrm{M}-\mathrm{N}$ \\
\hline 18 & $\begin{array}{l}\text { Non-IBD, Colon cancer } \\
\text { Adenocarcinoma }\end{array}$ & $\mathrm{F}$ & 84 & Figure $1 \mathrm{M}-\mathrm{N}$ \\
\hline 19 & Ulcerative colitis & M & 51 & Figure $1 \mathrm{M}-\mathrm{N}$ \\
\hline 110 & Ulcerative colitis & M & 39 & Figure $1 \mathrm{M}-\mathrm{N}$ \\
\hline 111 & Ulcerative colitis & $\mathrm{F}$ & 45 & Figure $1 \mathrm{M}-\mathrm{N}$ \\
\hline $\mid 12$ & Ulcerative colitis & $\mathrm{F}$ & 43 & Figure $1 \mathrm{M}-\mathrm{N}$ \\
\hline 113 & Ulcerative colitis & $\mathrm{F}$ & 41 & Figure $1 \mathrm{M}-\mathrm{N}$ \\
\hline 114 & Ulcerative colitis & M & 59 & Figure $1 \mathrm{M}-\mathrm{N}$ \\
\hline $\mid 15$ & Ulcerative colitis & $\mathrm{F}$ & 61 & Figure $1 \mathrm{M}-\mathrm{N}$ \\
\hline 116 & Ulcerative colitis & M & 58 & Figure $1 \mathrm{M}-\mathrm{N}$ \\
\hline 117 & Crohn's disease & M & 43 & Figure $1 \mathrm{M}-\mathrm{N}$ \\
\hline 118 & Crohn's disease & M & 49 & Figure $1 \mathrm{M}-\mathrm{N}$ \\
\hline $\mid 19$ & Crohn's disease & $F$ & 51 & Figure $1 \mathrm{M}-\mathrm{N}$ \\
\hline 120 & Crohn's disease & $\mathrm{F}$ & 37 & Figure $1 \mathrm{M}-\mathrm{N}$ \\
\hline 121 & Crohn's disease & M & 62 & Figure $1 \mathrm{M}-\mathrm{N}$ \\
\hline 122 & Crohn's disease & M & 41 & Figure $1 \mathrm{M}-\mathrm{N}$ \\
\hline 123 & Crohn's disease & $\mathrm{F}$ & 52 & Figure $1 \mathrm{M}-\mathrm{N}$ \\
\hline
\end{tabular}


Table S3. Alignment length and percent identity requirements for confidently calling eukaryotic virus families.

\begin{tabular}{|c|c|c|}
\hline Family & $\begin{array}{c}\text { Minimum Percent } \\
\text { ID }\end{array}$ & $\begin{array}{c}\text { Minimum Alignment } \\
\text { length in nt }\end{array}$ \\
\hline Herpesviridae & 70 & 80 \\
\hline Picornaviridae & 80 & 50 \\
\hline Papillomaviridae & 70 & 75 \\
\hline Coronaviridae & 70 & 100 \\
\hline Rhabdoviridae & 70 & 75 \\
\hline Orthomyxoviridae & 70 & 80 \\
\hline Anelloviridae & 70 & 75 \\
\hline Virgaviridae & & 80 \\
\hline
\end{tabular}


Table S4. qPCR primer sequences.

\begin{tabular}{|c|c|}
\hline Name & Sequence $\left(5^{\prime} \rightarrow 3^{\prime}\right)$ \\
\hline $\begin{array}{l}\text { Human TNF_F } \\
\text { Human TNF_R } \\
\text { Human IL6_F } \\
\text { Human IL6_R } \\
\text { Human IL10_F }\end{array}$ & $\begin{array}{c}\text { ATGAGCACTGAAAGCATGATCC } \\
\text { GAGGGCTGATTAGAGAGAGGTC } \\
\text { TCTCCACAAGCGCCTTCG } \\
\text { CTCAGGGCTGAGATGCCG } \\
\text { GACTTTAAGGGTTACCTGGGTTG }\end{array}$ \\
\hline $\begin{array}{l}\text { Human IL10_R } \\
\text { Human GAPDH_F } \\
\text { Human GAPDH_R }\end{array}$ & $\begin{array}{l}\text { TCACATGCGCCTTGATGTCTG } \\
\text { AGGGCTGCTTTAACTCTGGT } \\
\text { CCCCACTTGATTTTGGAGGGA }\end{array}$ \\
\hline Human IFNB_F & CATTACCTGAAGGCCAAGGA \\
\hline Human IFNB_R & CAATTGTCCAGTCCCAGAGG \\
\hline Human ZO1_F & CGGTCCTCTGAGCCTGTAAG \\
\hline Human ZO1_R & GGATCTACATGCGACGACAA \\
\hline Human Occludin_F & TCAGGGAATATCCACCTATCACTTCAG \\
\hline Human Occludin_R & CATCAGCAGCAGCCATGTACTCTTCAC \\
\hline Human IFNL1_F & CGCCTTGGAAGAGTCACTCA \\
\hline Human IFNL1_R & GAAGCCTCAGGTCCCAATTC \\
\hline Human CISH_F & GCTGTGCATAGCCAAGACCTT \\
\hline Human CISH_R & CTGGCATCTTCTGCAGGTGTT \\
\hline Human SOCS2_F & TCGGTCAGACAGGATGGTACT \\
\hline Human SOCS2_R & AGTTCCTTCTGGTGCCTCTTT \\
\hline Human TGF $\beta 1$ F & CACCGGAGTTGTGCGGCAGT \\
\hline Human TGF $\beta 1 \_R$ & GGCCGGTAGTGAACCCGTTGAT \\
\hline $\begin{array}{l}\text { Mouse Cish_F } \\
\text { Mouse Cish_R }\end{array}$ & $\begin{array}{l}\text { TTCGGGAATCTGGGTGGTACT } \\
\text { GGTGGGTGCTGTCTCGAACTA }\end{array}$ \\
\hline Mouse Isg15_F & TGAGAGCAAGCAGCCAGAAG \\
\hline Mouse Isg15_R & ACGGACACCAGGAAATCGTT \\
\hline Mouse II6-F & TGGGAAATCGTGGAAATGAG \\
\hline Mouse II6-R & CCAGTTTGGTAGCATCCATCA \\
\hline Mouse Gapdh_F & TGAGCAAGAGAGGCCCTATC \\
\hline Mouse Gapdh_R & AGGCCССTCСTGTTATTATG \\
\hline
\end{tabular}


Table S5. Pertinent information for commercial antibody reagents used for FACS analysis.

\begin{tabular}{cllc}
\hline Antibody & Supplier & Species & Cat \# \\
\hline CD45 (30-F11; Pacific Blue) & Biolegend & Mouse & 103101 \\
CD11b (clone M1/70; APC/Cy7) & Biolegend & Mouse & 101225 \\
CD11c (N418; PE) & Biolegend & Mouse & 117307 \\
Gr-1 (RB6-8C5; APC) & Biolegend & Mouse & 108411 \\
CX3CR1 (SA011F11; Brilliant Violet & Biolegend & Mouse & 149029 \\
785) & & & \\
F4/80 (BM8; Brilliant Violet 650) & Biolegend & Mouse & 123149 \\
CD103 (2E7; PerCP/Cy5.5) & Biolegend & Mouse & 121415 \\
MHCII (M5/114.15.2; Alexa Fluor & Biolegend & Mouse & 107621 \\
$700)$ & & &
\end{tabular}




\section{References}

1. J. M. Norman et al., Disease-specific alterations in the enteric virome in inflammatory bowel disease. Cell 160, 447-460 (2015).

2. S. R. Finkbeiner et al., Identification of a novel astrovirus (astrovirus VA1) associated with an outbreak of acute gastroenteritis. J Virol 83, 10836-10839 (2009).

3. D. Wang et al., Viral discovery and sequence recovery using DNA microarrays. PLoS Biol 1, E2 (2003).

4. M. Steinegger, J. Soding, MMseqs2 enables sensitive protein sequence searching for the analysis of massive data sets. Nat Biotechnol 35, 1026-1028 (2017).

5. A. Dobin et al., STAR: ultrafast universal RNA-seq aligner. Bioinformatics 29, 15-21 (2013).

6. $\quad$ S. Anders, P. T. Pyl, W. Huber, HTSeq--a Python framework to work with highthroughput sequencing data. Bioinformatics 31, 166-169 (2015).

7. M. D. Robinson, D. J. McCarthy, G. K. Smyth, edgeR: a Bioconductor package for differential expression analysis of digital gene expression data. Bioinformatics 26, 139$140(2010)$.

8. S. Anders et al., Count-based differential expression analysis of RNA sequencing data using R and Bioconductor. Nat Protoc 8, 1765-1786 (2013).

9. A. Subramanian et al., Gene set enrichment analysis: a knowledge-based approach for interpreting genome-wide expression profiles. Proc Natl Acad Sci U S A 102, 1554515550 (2005).

10. H. Huang et al., Fine-mapping inflammatory bowel disease loci to single-variant resolution. Nature 547, 173-178 (2017).

11. C. DeBoever et al., Medical relevance of protein-truncating variants across 337,205 individuals in the UK Biobank study. Nat Commun 9, 1612 (2018).

12. S. Kumar, J. E. Curran, D. C. Glahn, J. Blangero, Utility of Lymphoblastoid Cell Lines for Induced Pluripotent Stem Cell Generation. Stem Cells Int 2016, 2349261 (2016).

13. K. Tamminen et al., Intestinal Commitment and Maturation of Human Pluripotent Stem Cells Is Independent of Exogenous FGF4 and R-spondin1. PLoS One 10, e0134551 (2015).

14. Y. Takahashi et al., Reciprocal Inflammatory Signaling Between Intestinal Epithelial Cells and Adipocytes in the Absence of Immune Cells. EBioMedicine 23, 34-45 (2017).

15. J. Y. Yang et al., Enteric Viruses Ameliorate Gut Inflammation via Toll-like Receptor 3 and Toll-like Receptor 7-Mediated Interferon-beta Production. Immunity 44, 889-900 (2016).

16. U. Erben et al., A guide to histomorphological evaluation of intestinal inflammation in mouse models. Int J Clin Exp Pathol 7, 4557-4576 (2014).

17. E. Bolyen et al., Reproducible, interactive, scalable and extensible microbiome data science using QIIME 2. Nat Biotechnol 37, 852-857 (2019).

18. B. J. Callahan et al., DADA2: High-resolution sample inference from Illumina amplicon data. Nat Methods 13, 581-583 (2016).

19. K. Katoh, K. Misawa, K. Kuma, T. Miyata, MAFFT: a novel method for rapid multiple sequence alignment based on fast Fourier transform. Nucleic Acids Res 30, 3059-3066 (2002). 
20. M. N. Price, P. S. Dehal, A. P. Arkin, FastTree 2--approximately maximum-likelihood trees for large alignments. PLoS One 5, e9490 (2010).

21. C. Lozupone, R. Knight, UniFrac: a new phylogenetic method for comparing microbial communities. Appl Environ Microbiol 71, 8228-8235 (2005).

22. N. A. Bokulich et al., Optimizing taxonomic classification of marker-gene amplicon sequences with QIIME 2's q2-feature-classifier plugin. Microbiome 6, 90 (2018).

23. D. McDonald et al., An improved Greengenes taxonomy with explicit ranks for ecological and evolutionary analyses of bacteria and archaea. ISME J 6, 610-618 (2012).

24. N. Segata et al., Metagenomic biomarker discovery and explanation. Genome Biol 12, R60 (2011). 